\title{
Coarse-grained molecular dynamics: Nonlinear finite elements and finite temperature
}

\author{
Robert E. Rudd* \\ Lawrence Livermore National Laboratory, Condensed Matter Physics Div., L-045, Livermore, CA 94551 USA \\ Jeremy Q. Broughton \\ JPMorgan Chase Bank, New York, NY 10017 USA
}

(Dated: October 16, 2018)

\begin{abstract}
Coarse-grained molecular dynamics (CGMD) is a technique developed as a concurrent multiscale model that couples conventional molecular dynamics (MD) to a more coarse-grained description of the periphery. The coarse-grained regions are modeled on a mesh in a formulation that generalizes conventional finite element modeling (FEM) of continuum elasticity. CGMD is derived solely from the MD model, however, and has no continuum parameters. As a result, it provides a coupling that is smooth and provides control of errors that arise at the coupling between the atomistic and coarse-grained regions. In this article, we elaborate on the formulation of CGMD, describing in detail how CGMD is applied to anharmonic solids and finite temperature simulations. As tests of CGMD, we present in detail the calculation of the phonon spectra for solid argon and tantalum in 3D, demonstrating how CGMD provides a better description of the elastic waves than that provided by FEM. We also present elastic wave scattering calculations that show the elastic wave scattering is more benign in CGMD than FEM. We also discuss the dependence of scattering on the properties of the mesh. We introduce a rigid approximation to CGMD that eliminates internal relaxation, similar to the Quasicontinuum technique, and compare it to the full CGMD.

PACS numbers: 61.43.Bn, 02.70.Ns, 62.20.-x, 62.30.+d
\end{abstract}

\section{COUPLING OF LENGTH SCALES}

The Science of Materials is foremost a study of structure. Once structure is determined other important issues such as dynamics and kinetics may be addressed. Structure in materials is most effectively analyzed according to its length scale. Materials structure at different scales such as crystal structure, crystal defect structure, microstructure and macrostructure has led to the development of models at the atomic scale, nanoscale, microscale, and macroscale, not to mention the mesoscale and a vast array of other distinctions in scale. These models work because the physics at one scale decouples to a large extent from that at other scales, provided there exists a sufficient separation of scales. Then physical properties calculated at one scale may be passed to the next higher scale in a hierarchical approach that can be very effective 1.2

There are systems of interest that are inherently multiscale where the physics at one scale is strongly coupled to that at other scales $\frac{3}{3}$ Turbulence is an excellent example, where energy input through stirring at the macroscopic scale cascades down through vorticity across a range of length scales until it is ultimately dissipated at the shortest length scales. The size of the vortices varies continuously, and while there are length scales with distinct physics, the boundaries between them are blurred. As a result hierarchical models have been largely unsuccessful, and turbulence remains a hard problem. $\underline{\underline{4}}$ This situation is in marked contrast to low Reynolds number flow, in which the physics at small length scales can be encoded in a few parameters, which may be computed and then fed into simulations at a larger scale. In this way, it has been possible to start with ab initio electronic structure calculations of $\mathrm{H}_{2} \mathrm{O}$ and through a sequence of scales end up with a description of tides in Buzzard's Bay $\underline{\underline{5}}$

Many other examples of strongly coupled multiscale systems exist. Ironically, the advent of Nanoscience and the current focus on structures of one particular scale, the nanoscale, has led to the need to understand a class of strongly coupled multiscale systems. Consider epitaxial quantum dots, for example 6.7 .8 The quantum dot consists of a dome of semiconductor that forms during heteroepitaxy. The dome itself is typically a few nanometers to tens of nanometers across, but its size, shape and location are affected by the presence of other structures during growth, even those microns away. Another example is a Nano-Electro-Mechanical System (NEMS) resonator ${ }^{9.10,11.12}$ It consists of a semiconductor bar about $50 \mathrm{~nm}$ wide and a fraction of a micron long attached to the substrate at both ends. The bar itself is clearly nanoscale, and yet as it resonates, the oscillating strain fields extend far into the substrate. Of course, there are many other examples in metallurgy and solid state physics, as indicated above. Remarkably, similar effects are beginning to be appreciated in the study of soft materials, chemistry and even biology.

These systems are examples of what could be termed embedded nanomechanics 13 The mechanical properties of the nanoscale structure is clearly important, and these properties may be quite different from what would be predicted according to conventional macroscopic mechanics, but also important is the way the nanoscale structure is coupled to its surroundings. Embedded nanome- 


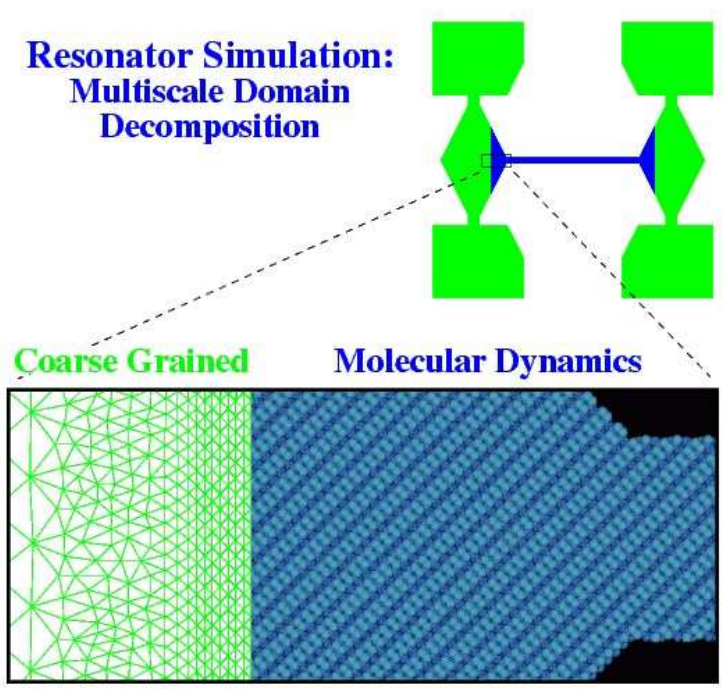

FIG. 1: (color online) Schematic diagram of a coarse-grained simulation of a NEMS silicon microresonator. ${ }^{4-6}$ The coarsegrained (CG) region comprises most of the volume, but the molecular dynamics (MD) region contains most of the simulated degrees of freedom. Each sphere shown in the MD region represents an atom. Note that the CG mesh is refined to the atomic scale where it joins with the MD lattice.

chanical structures are too small to be modeled reliably with conventional continuum elastic theory and finite elements, and too large to be modeled by conventional atomistics. Even in single crystals, sub-micron dynamical regions bounded by surfaces or interfaces are affected by Angstrom- and nano-scale physics which causes deviations from continuum elastic theory $\stackrel{14}{\Perp}$ dynamical regions larger than 0.1 micron cubed exceed the current limit of about one billion atoms for atomistic simulation of solids on a supercomputer $\stackrel{\underline{15}}{\underline{n}}$ The atomistic effects are compounded in materials with local defects or cracks that couple to long-range strain fields $\underline{16}$ The situation is not entirely intractable, however, because the most important atomistic effects are often localized to small regions of the system: surfaces, defects, regions of large deflection or internal strain, and regions of localized heating perhaps due to friction. The challenge is to develop a robust model for such an inhomogeneous system which captures the important atomistic effects without the prohibitive computational cost of a brute force atomistic simulation for the entire system. In this article we focus on the link between the micron scale and the nanoscale and develop a model, coarse-grained molecular dynamics (CGMD), 17 which bridges the disparate scales seamlessly.

The choice to use atomistic models at the finest resolution is motivated in some cases by the fact that the inherent length scale of the process of interest is the interatomic spacing and in other cases by the ability to derive interatomic potentials from quantum mechanics and hence built a model from first principles. Yet another motivation is that the processes of interest may be thermally activated, and molecular dynamics provides a means to simulate the thermal effects directly. Entropic and thermal effects are often paramount in soft matter systems, and in hard matter thermal activation is important in defect diffusion, the motion of dislocations in metals with high Peierls barriers and many nucleation phenomena. Temperature is important in other ways, too, such as in inducing phase transitions. Also the population of phonons increases with temperature, causing thermal expansion, changes (typically softening) in the elastic constants and dislocation drag at high strain rates. These are but a few well-known examples of the important role temperature plays, and thus in our development of multiscale models we search for methodologies capable of handling non-zero temperatures.

The variation of the strain field/atomic displacements in inhomogeneous solid systems suggests the use of different computational methodologies for different regions, as we mentioned above. The challenge is to meld them into a seamless, monolithic simulation. The first such proposal implemented a coupling between molecular dynamics $18.19(\mathrm{MD})$ and a finite element mode 20.21 (FEM) implementation of continuum elastic theory using stress consistency as the boundary condition at the interface ${ }^{22}$ More recently, a dynamical instability in the original formulation has been eliminated through the use of a mean force boundary condition together with uniform symplectic time evolution ${ }^{23}$ In both of these formulations, the same constitutive relation is used regardless of the size of the cells in the FEM mesh, leading to a discontinuity at the atomic limit.

At its heart the FEM description of such a system relies on the ability to improve the accuracy of the simulation by going to a finer mesh ${ }^{21} \mathrm{~A}$ mesh of varying coarseness is chosen, adaptively or by fiat, such that no single region contributes disproportionately to the error. These errors typically result from large strain, velocity, or other gradients which violate the discrete expression for the integral of the elastic energy density of a continuous medium. This approximation may be improved by mesh refinement. There is a limit, however. As the mesh size approaches the atomic scale, the constitutive equations have significant errors because the expression for the elastic energy does not represent localized bonds and the standard distributed mass expression for the kinetic energy does not account for the fact that essentially all of the mass is localized in the nuclei, at least four order of magnitude smaller than the interatomic spacing. At this point, the physics of the governing equations is wrong, and further mesh refinement does not help.

The approach of Refs. 22 and 23 to improve this situation replaces the FEM equations of motion on regions of the mesh that are atomic-sized with MD equations of motion and implements a hand shaking between the MD and FEM regions. Although this technique is remarkably successful, the union is not perfectly seamless. In the FEM 
cells approaching the atomic limit, the energy density varies smoothly within each cell, whereas on the other side of the interface, the MD energy is effectively localized to interatomic bonds. The short-wavelength modes of the system are able to probe this discrepancy, leading to errors that grow with the wavenumber.

The quasicontinuum technique ${ }^{24.25 .26}$ offers another approach to this problem. It is a zero temperature relaxation technique in which the elastic energy used in the FEM region is computed by applying the FEM interpolated displacement field (through the Cauchy-Born rule) to a reference system of atoms interacting by $\mathrm{MD}$ forces. This is a very nice idea, but it has a number of difficulties in practice. The atoms in the reference system are taken to be at their zero temperature locations-no fluctuations are allowed. Thus, many degrees of freedom are summarily set to zero (although finite temperature versions of the quasicontinuum technique are currently under development ${ }^{27}$ ). Also, the implementation of the quasicontinuum technique suffers from discontinuities ("ghost forces") due to the mismatch of the displaced reference systems from cell to cell and non-locality of atomic bonding ${ }^{28}$

Other concurrent multiscale models have been proposed recently. There are several nice review articles on this subject to which we direct interested readers ${ }^{26.28 .29}$ The relationships of several multiscale models to CGMD are of particular note. A finite temperature dynamical model based on renormalization group ideas has been proposed by Curtarolo and Ceder ${ }^{30}$ The finite temperature coarse-graining approach based on Monte Carlo calculations has been developed recently by Wu et al ${ }^{31}$ The bridging scale decomposition is another approach for coupling atomistic and continuum simulations due to Wagner and Liu that provides a coupling between FEM and atomistics that does not require the FEM mesh to be refined to the atomic leve ${ }^{32}$, and it has been applied to finite temperature simulations by Park et al ${ }^{33}$ The projection techniques of the bridging scale decomposition and the ensemble averages that they use are closely related to the techniques of CGMD introduced earlier. Also, we note that the assumption that no defect (dislocation) propagation takes place from an atomistic region into a finite element or coarse-grained region has been relaxed through the development of the CADD method, albeit so far just in two dimensions $\underline{34}^{34}$

We have proposed a replacement for conventional finite elements suitable for a mesh which is atomic sized in some regions $\stackrel{17}{17}$ This technique, CGMD, effectively provides the scale-dependent constitutive equations needed at the interface. In the atomic limit it is guaranteed to reproduce the atomistic equations of motion. This enables MD regions to be coupled seamlessly to regions of generalized FEM, bringing the full power of MD to bear on important parts of the system without the computational overhead of MD in other large, but physically less complex regions. The CGMD procedure is based on a statistical coarse graining prescription. While various aspects of CGMD have been introduced previously, this is the first article to present the model in great detail.

This kind of multi-scale simulation poses a number of challenges. First, the model must have a well-behaved, physical response to stationary strain fields, slowly varying in position, that extend into the CG region-there should be no ghost forces. Second, the model must have sensible thermodynamics in equilibrium. The effect of short-wavelength modes cannot be set to zero unless their energy is well above the thermal energy. Third, the system must have realistic dynamics, free of pathologies due to influences in the central MD region propagating out to unphysical interfaces, reflecting and propagating back into the central region to cause unphysical effects. Fourth, the model should exhibit well-behaved nonequilibrium thermodynamics, with a sensible response when low-lying modes are driven out of equilibrium. Finally, the methodology needs to be amenable to a practical implementation in terms of being able to utilize the broad spectrum of MD models in use, including many-body interatomic potentials that extend beyond nearest neighbors, computationally efficient domain decompositions for parallel distributed memory computers, visualization, etc. In this article we describe in detail how CGMD is implemented in order to meet these challenges.

In particular, we provide the first detailed description of the way CGMD may be applied to anharmonic solids and finite temperature simulations. Previously, we have described how CGMD is formulated for harmonic lattices ${ }^{17}$, including finite temperature contributions. We have also indicated how anharmonic effects are included, but the details have not been given. We give the details here, and explore their implications. We also introduce a rigid approximation to CGMD that eliminates internal relaxation, simplifying the formulation and reducing the computation cost of CGMD. We examine the physical difference between CGMD and its rigid approximation. As tests of CGMD, the calculation of the phonon spectra for solid argon and tantalum in 3D are presented. These calculations provide a test of the quality of the representation of elastic wave energetics. We also present elastic wave scattering calculations, a test of how CGMD behaves on an irregular mesh. Whenever possible we present analytic formulas that contain a wealth of information about the performance of CGMD in as much generality as possible (explicitly showing dependence on the interatomic potential, atomic masses, crystal lattice and mesh structure). Of course, realistic simulations involve numerical assembly and integration of the CGMD equations of motion.

\section{COARSE GRAINING PRESCRIPTION}

Consider a system of MD atoms in a solid, crystalline or amorphous, and a coarse-grained (CG) mesh partitioning the solid into cells (cf. Fig. 1). The mesh size may vary, so that in important regions a mesh node is assigned 
to each equilibrium atomic position, whereas in other regions the cells contain many atoms and the nodes need not coincide with atomic sites. CGMD offers a way to reduce the atomistic coordinates to a much smaller set of degrees of freedom associated with the displacement field at the nodes of the CG mesh, and the equations of motion for this mean displacement field. In particular, the energy functional for the CG system is defined as a constrained ensemble average of the atomistic energy under fixed thermodynamic conditions. The equations of motion are Hamilton's equations for this conserved energy functional and in principle additional random and dissipative forces due to fluctuations.

The classical ensemble must obey the constraint that the position and momenta of the atoms are consistent with the mean displacement and momentum fields. Let the displacement of atom $\mu$ be $\mathbf{u}_{\mu}=\mathbf{x}_{\mu}-\mathbf{x}_{\mu 0}$ where $\mathbf{x}_{\mu 0}$ is its equilibrium position. The displacement of mesh node $j$ is a weighted average of the atomic displacements

$$
\mathbf{u}_{j}=\sum_{\mu} f_{j \mu} \mathbf{u}_{\mu}
$$

where $f_{j \mu}$ is a weighting function, related to the microscopic analog of FEM interpolating functions below. An analogous relation applies to the momenta $\mathbf{p}_{\mu}$. Since the nodal displacements are fewer or equal to the atomic positions in number, fixing the nodal displacements and momenta does not (necessarily) determine the atomic coordinates entirely. Some subspace of phase space remains, corresponding to degrees of freedom that are missing from the mesh. We define the CG energy as the average energy of the canonical ensemble on this constrained phase space:

$$
\begin{aligned}
& E\left(\mathbf{u}_{k}, \dot{\mathbf{u}}_{k}\right)=\left\langle H_{M D}\right\rangle_{\mathbf{u}_{k}, \dot{\mathbf{u}}_{k}} \\
&=\int d \mathbf{x}_{\mu} d \mathbf{p}_{\mu} H_{M D} e^{-\beta H_{M D}} \Delta / Z \\
& Z\left(\mathbf{u}_{k}, \dot{\mathbf{u}}_{k}\right)=\int d \mathbf{x}_{\mu} d \mathbf{p}_{\mu} e^{-\beta H_{M D}} \Delta, \\
& \Delta=\prod_{j} \delta\left(\mathbf{u}_{j}-\sum_{\mu} \mathbf{u}_{\mu} f_{j \mu}\right) \delta\left(\dot{\mathbf{u}}_{j}-\sum_{\mu} \frac{\mathbf{p}_{\mu} f_{j \mu}}{m_{\mu}}\right)
\end{aligned}
$$

where $\beta=1 /(k T)$ is the inverse temperature, $Z$ is the partition function and $\delta(\mathbf{u})$ is a three-dimensional delta function. The delta functions enforce the mean field constraint (11). Note that Latin indices, $j, k, \ldots$, denote mesh nodes and Greek indices, $\mu, \nu, \ldots$, denote atoms. The energy (3) is computed below (Eq. (29)).

When the mesh nodes and the atomic sites are identical, $f_{j \mu}=\delta_{j \mu}$ and the CGMD equations of motion agree with the atomistic equations of motion ${ }^{35}$ As the mesh size increases some short-wavelength degrees of freedom are not supported by the coarse mesh. These degrees of freedom are not neglected entirely, because their thermodynamic average effect has been retained. This approximation is expected to be good provided the system is initially in thermal equilibrium, and changes to the system would only produce adiabatic changes in the missing degrees of freedom. In particular, the relaxation time of those degrees of freedom should be fast compared to the driving forces in the $\mathrm{CG}$ region. As long as this condition is satisfied, the long wavelength modes may be driven out of equilibrium without problems 36

We have written the CG energy as an internal energy, a function of the entropy, $S$, rather than the temperature. This is designed for systems in which the short wavelength modes change adiabatically. This is a good approximation, for example, when long wavelength elastic waves propagate through a solid in the linear regime at finite temperature $\frac{37.38}{}$ In other systems, the short wavelength modes may be in contact with a heat bath, so that their evolution is isothermal rather than isentropic. For example, the electron gas in metals can act as a heat bath on time scales longer than the thermal relaxation time. Then the Helmholz free energy,

$$
F\left(\mathbf{u}_{k}, \dot{\mathbf{u}}_{k}\right)=-k T \log Z,
$$

should be used rather than the internal energy. In this case, the ensemble average behavior of the CG collective modes is exactly the same as that of the corresponding averaged atomic modes in the underlying atomistic system:

$$
\begin{aligned}
\left\langle\mathbf{u}_{j_{1}} \cdots \mathbf{u}_{j_{n}}\right\rangle= & \int d \mathbf{u}_{j} d \dot{\mathbf{u}}_{j}\left(\mathbf{u}_{j_{1}} \cdots \mathbf{u}_{j_{n}}\right) e^{-\beta F} \\
= & f_{j_{1} \mu_{1}} \cdots f_{j_{n} \mu_{n}} \times \\
& \quad \int d \mathbf{u}_{\mu} d \mathbf{p}_{\mu} \mathbf{u}_{\mu_{1}} \cdots \mathbf{u}_{\mu_{n}} e^{-\beta H_{M D}},
\end{aligned}
$$

which follows from plugging in the expression for $F$ (6) and (4) into (7) and integrating the delta functions ${ }^{39}$ This equation shows the equivalence of all unnormalized correlation functions, but since the partition functions (zero point functions) are identical, the normalized correlation functions are the same, as well. The emergence of the canonical distribution in other cases requires a treatment of thermal relaxation processes (cf. Section $\mathrm{V}$ ). It should be noted that even in the isothermal ensemble the faithfulness of correlation functions applies only to equaltime correlation functions at equilibrium, and consideration of dissipative processes are needed to reproduce interesting correlation functions such as the autocorrelation function $\left\langle\dot{u}_{i}(0) \dot{u}_{i}(t)\right\rangle$ associated with the fluctationdissipation theorem.

To end this section, we note that the definition of the CGMD energy may appear to neglect the well-known quantum mechanical contributions to lattice dynamics. Phonons are bosons, after all, and they should obey Bose-Einstein statistics. The definition of the CGMD energy (31) is clearly a classical expression based on Boltzmann statistics. To what extent can it be expected to be valid? The reason the classical expression is valid for most of the conceivable applications of CGMD is that the Bose-Einstein distribution most strongly affects the 
lowest states; i.e., exactly the states that are retained explicitly in the CGMD Hamiltonian. The higher energy states have low occupation in equilibrium, and are not affected significantly by strong quantum effects such as Bose condensation. The CGMD Hamiltonian is therefore expected to be a good description of the coarsegrained system. It may be necessary to use a path integral, or other quantum mechanical version, of MD to treat the retained degrees of freedom at sufficiently low temperature,$\stackrel{40}{,}$ but the internal degrees of freedom are described well by Eq. (31).

\section{SHAPE FUNCTIONS}

In addition to the general framework we have presented for CGMD, a specific choice of the weighting functions is required for calculations. They result from the introduction of a set of shape functions $\left\{N_{j}(\mathbf{x})\right\}_{j=1}^{N_{\text {node }}}$ on the mesh from which the interpolated fields are constructed. The shape functions have the following properties:

$$
\begin{aligned}
& \text { i. } N_{j}\left(\mathbf{x}_{k}\right)=\delta_{j k}, \\
& \text { ii. } \sum_{j=1}^{N_{\text {node }}} N_{j}(\mathbf{x})=1,
\end{aligned}
$$

iii. $\mathrm{C}^{0}$ continuity.

The first property states that the functions are normalized and local on the mesh nodes, $\mathbf{x}_{k}$. The second states that the functions form a partition of unity, so the center of mass mode is represented. The third states that the functions are continuous but their derivatives need not be. This continuity guarantees that the elastic energy, proportional to an integral of the square of the strain $(\partial \mathbf{u})_{\text {sym }}$, is well-behaved in the continuum limit. The interpolated displacement field is then defined by $\mathbf{u}(\mathbf{x})=\sum \mathbf{u}_{j} N_{j}(\mathbf{x})$. Often there are additional considerations, such as the need to refine the mesh onto a particular crystal lattice at the MD/CG interface ${ }^{41}$

Given any set of atomic displacements we can find the displacement field represented on the CG mesh which best fits these data in the least squares sense:

$$
\chi^{2}=\sum_{\mu}\left|\mathbf{u}_{\mu}-\sum_{j} \mathbf{u}_{j} N_{j \mu}\right|^{2},
$$

where $N_{j \mu}=N_{j}\left(\mathbf{x}_{\mu 0}\right)$. This $\chi^{2}$ error is minimized by

$$
\begin{aligned}
\mathbf{u}_{j} & =\sum_{\mu} f_{j \mu} \mathbf{u}_{\mu} \\
f_{j \mu} & =\sum_{k}\left(\sum_{\nu} N_{j \nu} N_{k \nu}\right)^{-1} N_{k \mu}
\end{aligned}
$$

[cf. (11)]. This equation defines the weighting function $f_{j \mu}$ of (11) in terms of the interpolating function $N_{j}(\mathbf{x})$. We note that recently this relationship introduced in CGMD has been generalized for use in the bridging scale, and other $\mathrm{L}^{2}$ projection techniques ${ }^{29}$

The formulation we have described is appropriate to retain the low-lying acoustic phonon modes in the coarsegrained system. In some cases it is desirable to retain the long-wavelength optical phonons, as well. For example, in the study of III-V epitaxial quantum dots, internal relaxation of the zinc blende structure in the strained dots leads to important changes in the optical spectra of the dots ${ }^{42}$ If it is important to model the optical phonons or to capture the internal relaxation in a crystal lattice with a multiple-atom basis, each interpolation function should carry a band index, $a$, in addition to the nodal index, $j$ : $N_{j}^{(a)}(\mathbf{x})$. Then the basis requirements are somewhat different. The functions should be local and normalized within each band. They should be $\mathrm{C}^{0}$ continuous apart from variations with the unit cell. And they should form a generalization of the partition of unity. In particular, the requirement of forming a partition of unity is the requirement that uniform displacement of the system be represented in the basis of shape functions. That translation invariance is responsible for the $\mathbf{k}=0$ acoustic-mode phonons having zero energy. We generalize the partition of unity requirement to the requirement that all of the $\mathbf{k}=0$ phonon, both acoustic and optical, be represented. In particular, denote the displacement associated with the $\mathbf{k}=0$ phonon for band $a$ as $\mathbf{u}_{\mu}^{(a)}$, normalized such that

$$
\sum_{\mu \in \text { unit cell }}\left|\mathbf{u}_{\mu}^{(a)}\right|^{2}=N_{\text {basis }}
$$

where $N_{\text {basis }}$ is the number of atoms in the Wigner-Seitz unit cell. Then the shape functions can be defined as

$$
N_{j}^{(a)}\left(\mathbf{x}_{\mu 0}\right)=\mathbf{u}_{\mu}^{(a)} N_{j}\left(\mathbf{x}_{\mu 0}\right)
$$

where $N_{j}(\mathbf{x})$ is a conventional shape function, such as linear interpolation. The generalized partition of unity requirement is that

$$
\sum_{j} N_{j}^{(a)}\left(\mathbf{x}_{\mu 0}\right)=\mathbf{u}_{\mu 0}^{(a)}
$$

Note that in the case of a monatomic unit cell this shapefunction basis is a linear combination of the shape functions we have discussed above. In that case $\mathbf{u}_{\mu}^{(a)}$ is the same for all lattice sites, and the orthonormal vectors corresponding to the three acoustic-mode phonons span three-dimensional space. We do not discuss an example of a polyatomic CGMD including optical phonons explicitly, but the CGMD formalism continues to work. It should be emphasized, however, that even in polyatomic materials this band-index extension may not be needed to capture the mechanical response of interest. 


\section{THE CGMD HAMILTONIAN}

We now turn to the calculation of the CGMD energy.

\section{A. Harmonic Lattices}

The CG energy (3) may be computed in closed form using analytic techniques in the case of a harmonic lattice. The expression was originally presented in Ref. 17. We take the form of the atomistic Hamiltonian to be

$$
H_{M D}=\sum_{\mu} \frac{\mathbf{p}_{\mu}^{2}}{2 m_{\mu}}+\sum_{\mu} E_{\mu}^{\mathrm{coh}}+\sum_{\mu, \nu} \frac{1}{2} \mathbf{u}_{\mu} \cdot D_{\mu \nu} \mathbf{u}_{\nu}
$$

where $E_{\mu}^{\mathrm{coh}}$ is the cohesive energy of atom $\mu$ and $D_{\mu \nu}$ is the dynamical matrix. It acts as a tensor on the components of the displacement vector at each site. We reexpress the CG energy (3) using a parametric derivative of the $\log$ of the constrained partition function (4),

$$
E\left(\mathbf{u}_{k}, \dot{\mathbf{u}}_{k}\right)=-\partial_{\beta} \log Z\left(\mathbf{u}_{k}, \dot{\mathbf{u}}_{k} ; \beta\right)
$$

and we introduce the Fourier transform representation of the delta function (a form of Lagrange multiplier) to simplify the constraint (5)

$$
\begin{aligned}
\Delta= & \int\left(\frac{d \lambda}{2 \pi}\right)^{3 N_{\text {node }}} e^{i \lambda_{j} \cdot\left(\mathbf{u}_{j}-f_{j \mu} \mathbf{u}_{\mu}\right)} \times \\
& \int\left(\frac{d \lambda^{\prime}}{2 \pi}\right)^{3 N_{\text {node }}} e^{i \lambda_{k}^{\prime} \cdot\left(\dot{\mathbf{u}}_{k}-f_{k \nu} \mathbf{p}_{\nu} / m_{\nu}\right)},
\end{aligned}
$$

where here, and in what follows unless stated otherwise, the repeated indices are summed. Expressed in this way, the constrained partition function for the harmonic lattice is a Gaussian integral. The complicated domain of integration in (3) resulting from the constraints is replaced by a simple domain plus some extra integrals. This standard technique gives an expression which may be evaluated in closed form.

For pedagogical purposes we present the calculation of the CG potential energy here in some detail. The calculations of each of the other CG energy terms, the CG kinetic and anharmonic potential terms, follow the same basic approach. It may be helpful therefore to present the calculation of the $\mathrm{CG}$ harmonic potential energy in detail, and readers who are not interested in this algebra may skip ahead to the next paragraph at Eq. (29).

In order to get a closed-form expression for the CG potential energy, we make use of the well-known exact formula for the integral over all space of a Gaussian times an arbitrary polynomial prefactor. In the interest of compact notation, we combine the atomic and spatial indices and consider the displacements and the dynamical ma-

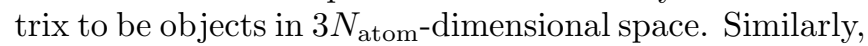
we take all $\mathrm{CG}$ variables to live in $3 N_{\text {node-dimensional }}$ space. The form for the Gaussian integral is known for these high dimensional spaces, and we evaluate the integral (4) through two successive Gaussian integrations: first an integral over the MD phase space and then an integral over the Lagrange multiplier space. Using (16), we need to calculate the CG partition function. It factorizes into kinetic and potential parts, $Z=Z_{\text {kin }} Z_{\text {pot }}$. We focus on the potential energy part of the $\mathrm{CG}$ partition function:

$$
Z_{\text {pot }}\left(u_{k} ; \beta\right)=\int d u \int d \lambda e^{-\frac{1}{2} \beta u_{\mu} D_{\mu \nu} u_{\nu}+i \lambda_{j}\left(u_{j}-f_{j \mu} u_{\mu}\right)}
$$

where $d u=(d u)^{3 N_{\text {atom }}}$ and $d \lambda=\left(\frac{d \lambda}{2 \pi}\right)^{3 N_{\text {node }}}$. Here, and throughout this derivation, we suppress the cohesive energy by choosing the zero of energy such that the cohesive energy is zero; we then restore the cohesive energy in the final formulas. We first compute the integral over $d u$ by completing the square in the argument of the exponential. Let

$$
\tilde{u}_{\mu}=u_{\mu}-i D_{\mu \nu}^{-1} f_{j \nu} \lambda_{j} / \beta
$$

where we assume that the matrix inverse $D_{\mu \nu}^{-1}$ exists after a suitable regularization to deal with the zero eigenvalues (we will return to this point). The shift (19) leaves the measure $d u$ invariant, so

$$
\begin{aligned}
Z_{\text {pot }}\left(u_{k} ; \beta\right)=\int d \tilde{u} e^{-\frac{1}{2} \beta \tilde{u}_{\mu} D_{\mu \nu} \tilde{u}_{\nu}} \times \\
\\
\int d \lambda e^{i \lambda_{j} u_{j}+\frac{1}{2} \lambda_{j} f_{j \mu} D_{\mu \nu}^{-1} f_{k \nu} \lambda_{k} / \beta}
\end{aligned}
$$

where the integral has now split into two independent factors. The Gaussian integral over $d \tilde{u}$ is elementary 43 :

$$
\begin{aligned}
\int d \tilde{u} e^{-\beta \tilde{u}_{\mu} D_{\mu \nu} \tilde{u}_{\nu}} & =\left(2 \pi \beta^{-1}\right)^{3 N_{\text {atom }} / 2}\left(\operatorname{det}^{\prime} D\right)^{-1 / 2} \\
& =C_{1} \beta^{-3 N_{\text {atom }} / 2}
\end{aligned}
$$

where we have used $\beta^{-1}=k T$ and det $^{\prime}$ denotes the determinant without the zero eigenvalues. On the second line we note the simple power-law dependence on $\beta$; the constant factor $C_{1}$ is ultimately irrelevant. In order to simplify notation, we define $K_{j k}=\left(f_{j \mu} D_{\mu \nu}^{-1} f_{k \nu}\right)^{-1}$, where again a suitable regularization is implied on the righthand side. Then we have

$$
\begin{aligned}
Z_{\text {pot }}\left(u_{k} ; \beta\right)=C_{1} \beta^{-3 N_{\text {atom }} / 2} \times \\
\\
\qquad d \lambda e^{i \lambda_{j} u_{j}+\frac{1}{2} \lambda_{j} K_{j k}^{-1} \lambda_{k} / \beta}
\end{aligned}
$$

Now we compute the next integral, again by introducing a suitable shift in the variables:

$$
\tilde{\lambda}_{j}=\lambda_{j}-i \beta K_{j k} u_{k}
$$

so that

$$
\begin{aligned}
Z_{\text {pot }}\left(u_{k} ; \beta\right)= & C_{1} \beta^{-3 N_{\text {atom }} / 2} \times \\
& \int d \tilde{\lambda} e^{\frac{1}{2} \tilde{\lambda}_{j} K_{j k}^{-1} \tilde{\lambda}_{k} / \beta} e^{-\frac{1}{2} \beta u_{j} K_{j k} u_{k}}
\end{aligned}
$$


Again the Gaussian integral is elementary

$$
\int d \tilde{\lambda} e^{\frac{1}{2} \tilde{\lambda}_{j} K_{j k}^{-1} \tilde{\lambda}_{k} / \beta}=C_{2} \beta^{3 N_{\text {node }} / 2}
$$

where only the dependence on $\beta$ is relevant. 44 Finally, we have the expression we need:

$$
\begin{gathered}
Z_{\text {pot }}\left(u_{k} ; \beta\right)=C_{1} C_{2} \beta^{-3\left(N_{\text {atom }}-N_{\text {node }}\right) / 2} \times \\
e^{-\frac{1}{2} \beta u_{j} K_{j k} u_{k}}
\end{gathered}
$$

The CG (harmonic) potential energy is then

$$
\begin{aligned}
E_{\text {pot }}\left(u_{k}\right) & =-\partial_{\beta} \log Z_{\text {pot }} \\
& =\frac{3}{2}\left(N_{\text {atom }}-N_{\text {node }}\right) k T+\frac{1}{2} u_{j} K_{j k} u_{k}
\end{aligned}
$$

This expression shows that the CG potential energy is exactly given by the sum of two contributions. First, each degree of freedom that has been integrated out contributes a thermal energy of $\frac{1}{2} k T$ to the potential energy. And second, the remaining CG degrees of freedom experience a harmonic potential of the form $\frac{1}{2} u_{j} K_{j k} u_{k}$. The calculation of the kinetic energy is essentially identical in form, but a bit more simple due to the diagonal mass matrix. We note that while this derivation is mathematically elegant, it finesses many subtleties in a way that may not give the reader complete confidence in the derivation; e.g. we have ignored the fact that the dynamical matrix is singular and we have not been very careful about the values of the irrelevant constants $C_{1}$ and $C_{2}$. We present a more careful derivation in Appendix A that has the distinct benefit that the reason for, and extent of, the non-locality of the stiffness matrix is readily apparent. We now return to the CG energy, and once again separate the spatial and nodal indices for the following.

The full CG energy (16) for a monatomic harmonic solid of $N_{\text {atom }}$ atoms coarse grained to $N_{\text {node nodes is }}$ then given by

$$
E\left(\mathbf{u}_{k}, \dot{\mathbf{u}}_{k}\right)=U_{\mathrm{int}}+\frac{1}{2} \sum_{j, k}\left(M_{j k} \dot{\mathbf{u}}_{j} \cdot \dot{\mathbf{u}}_{k}+\mathbf{u}_{j} \cdot K_{j k} \mathbf{u}_{k}\right),
$$

where the contribution of the internal degrees of freedom is

$$
U_{\text {int }}=N_{\text {atom }} E^{\text {coh }}+3\left(N_{\text {atom }}-N_{\text {node }}\right) k T .
$$

$M_{j k}$ and $K_{j k}$ are defined as follows. The mass matrix is

$$
\begin{aligned}
M_{j k} & =\left(\sum_{\mu} f_{j \mu} m_{\mu}^{-1} f_{k \mu}\right)^{-1} \\
& =m \sum_{\mu} N_{j \mu} N_{k \mu} \quad \text { (monatomic), }
\end{aligned}
$$

where the second line applies to monatomic solids with atomic mass $m$. This may be expressed in matrix notation as

$$
\begin{aligned}
M_{j k} & =\left[\left(N N^{T}\right)\left(N M_{M D}^{-1} N^{T}\right)^{-1}\left(N N^{T}\right)\right]_{j k} \\
& =m\left(N N^{T}\right)_{j k} \quad \text { (monatomic) }
\end{aligned}
$$

where the MD mass matrix is $M_{\mu \nu}^{M D}=m_{\mu} \delta_{\mu \nu}$. Note that each of the quantities in parentheses () in Eq. (33) is an $N_{\text {node }} \times N_{\text {node }}$ square matrix.

At times, it may be desirable to use a diagonal approximation of the mass matrix, often called the lumped mass matrix in the FEM literature. In CGMD it is given by ${ }^{3}$

$$
M_{i j}^{\text {lump }}=\delta_{i j} \sum_{\mu} N_{j \mu} m_{\mu}
$$

The CGMD stiffness matrix is given formally by

$$
\begin{aligned}
K_{j k} & =\left(\sum_{\mu \nu} f_{j \mu} D_{\mu \nu}^{-1} f_{k \nu}\right)^{-1} \\
& =\left[\left(N N^{T}\right)\left(N D^{-1} N^{T}\right)^{-1}\left(N N^{T}\right)\right]_{j k},
\end{aligned}
$$

where each of the entries on the second line is a matrix. The inverses in (31), (33), (36) and (37) are matrix inverses.

Remarkably, there is another way to write the CGMD mass and stiffness matrices that does not require two inverses. These forms are derived in Appendix $\mathrm{A}$ and the notation is explained there. In particular, they are given by

$$
\begin{aligned}
& K_{j k}=N_{j \mu} D_{\mu \nu} N_{k \nu}-D_{j \mu}^{\times} \tilde{D}_{\mu \nu}^{-1} D_{k \nu}^{\times} \\
& M_{j k}=N_{j \mu} m_{\mu} N_{k \mu}-M_{j \mu}^{\times} \tilde{M}_{\mu \nu}^{-1} M_{k \nu}^{\times}
\end{aligned}
$$

We do not currently know of an easy way to show the equivalence of Eqs. (36) and (38), but we have checked that they are numerically equal. Each form has its preferred uses. In the calculation of the CGMD spectra and any other application in which the stiffness matrix in reciprocal space is needed, Eq. (36) is advantageous. It is formally simpler, but it suffers from requiring two inverses and from formal singularities due to the zero modes of the dynamical matrix. Both of these drawbacks disappear in reciprocal space, where the dynamical matrix is diagonal (in the Fourier transform of the nodal indices). On the other hand, Eq. (38) is well defined, and it only requires one inverse. Typically, the inverse in the atomic indices is taken in reciprocal space making use of the perfect crystal space group symmetry, whereas the second inverse (36) is in the nodal indices for which reciprocal space offers an advantage only in special cases where the mesh is uniform. For irregular meshes, Eq. (38) is preferred.

Consider the form of the second expression for the stiffness matrix (38). The first term represents a form of coarse graining in which each atom is forced to be exactly at the position defined by the interpolation function. Within the context of CGMD, we will refer to the approximation where the other terms are neglected as the rigid approximation. To be precise,

$$
P_{\mu \nu}^{\perp} \rightarrow 0 \quad \text { Rigid Approximation }
$$

by which we mean that $P_{\mu \nu}^{\perp}$, defined in Eq. A7 is set to zero in all of the subsequent CGMD formulas. For 
instance, both $D_{j \nu}^{\times}$and $M_{j \nu}^{\times}$vanish in the rigid approximation, so only the first term survives in Eqs. (38) and (39) .

Now let us consider the second term in the stiffness matrix (38). This kind of term has not been discussed in the context of concurrent multiscale modeling previously, and it is very interesting. It is in the form of a lattice Green function contribution to the stiffness. According to our principle of microscopic-macroscopic correspondence, the atomistic degrees of freedom are assumed to be a best fit to the coarse-grained degrees of freedom. Even at zero temperature, this requirement does not necessarily mean that the atoms will be position exactly where the interpolation function would put them. Instead, they typically relax to a lower energy configuration. This relaxation in the short wavelength degrees of freedom introduces a non-local coupling between the coarse-grained degrees of freedom through the Green function $\tilde{D}_{\mu \nu}^{-1}$. The appearance of a Green function in a relaxation problem is natural. Consider a system governed by the elastic energetics $E=\frac{1}{2} u_{\mu} D_{\mu \nu} u_{\nu}-f_{\mu} u_{\mu}$. The minimal energy state is $u_{\mu}=D_{\mu \nu}^{-1} f_{\mu}$ with the energy $E_{\min }=-\frac{1}{2} u_{\mu} D_{\mu \nu}^{-1} u_{\nu}$. The Green function thus arises naturally in the energy of the relaxed state. In CGMD, it is the internal modes that can relax, and so it is the Green function of these internal modes that enters the CGMD Hamiltonian and introduces the non-locality. This kind of weak non-locality is not present in finite element modeling, but it is entirely physical. In fact, continuum formulations of nonlocality in elasticity have been introduced to account for size effects in dislocations, crack tips and other nanoscale structures $\stackrel{45.46}{ }$ It may be possible to neglect the nonlocality for a particular application, but it is real and it arises naturally in CGMD.

The energy (29) contains terms representing the average kinetic and potential energies, plus the thermal energy term expected from the equipartition theorem for the modes that have been integrated out. As mentioned above, this Hamiltonian continues to work for polyatomic solids, in which the optical modes may be coarse grained in various ways to represent different physics.

\section{B. No Ghost Forces}

One goal of concurrent multiscale modeling is to have the atoms in the atomistic region behave as closely as possible to the way they would if the atomistics extended throughout the system. Deviations from this ideal have been termed ghost forces. Some deviation is inevitable, but two kinds of deviation have received particular attention as pathologies. Both are at zero temperature. First, if the displacement with respect to the equilibrium lattice is zero throughout the system, then the atoms at the interface should experience no force. Second, if the displacement corresponds to uniform strain and the uniformly strained atomistic system is in equilibrium, then the atoms at the interface in the concurrent multiscale model should experience no force (See the recent review article by Curtin and Miller ${ }^{28}$ ).

CGMD does not suffer from the first type of ghost force, as can be seen immediately from the absence of terms linear in $\mathbf{u}_{j}$ in Eq. (29). The second type of ghost force is also absent from CGMD, provided the strain is admissible in the space of shape functions. This property should be clear from the construction of CGMD, where if the best fit interpolation function reproduces the uniform strain at zero temperature, the delta functions impose that the CGMD energy agree with the MD energy exactly.

To be more precise, the strained state described by the underlying atomistic displacement $\mathbf{u}_{\mu}$ is admissible if there exists a set of nodal displacements $\mathbf{u}_{j}$ such that

$$
\begin{aligned}
\mathbf{u}_{\mu} & =\sum_{j} \mathbf{u}_{j} N_{j}\left(x_{\mu 0}\right) \\
& =\mathbf{u}_{\nu} f_{j \nu} N_{j \mu} \\
& =P_{\mu \nu}^{C G} \mathbf{u}_{\nu}
\end{aligned}
$$

where we have used Eq. (10) in the second line to express the best-fit $\mathbf{u}_{j}$ in terms of $\mathbf{u}_{\mu}$. The matrix $P_{\mu \nu}^{C G}$, defined in Appendix $\mathrm{A}$ in Eq. A6 , must act as the identity on $\mathbf{u}_{\nu}$. We assume that the uniformly strained atomistic system is in equilibrium, and hence $D_{\mu \nu} \mathbf{u}_{\nu}=0$. We calculate the force on node $i$ (which may be an atom at the interface) using Eqs. (38) and (10) as

$$
\begin{aligned}
-K_{i j} \mathbf{u}_{j} & =-N_{i \mu} D_{\mu \nu} N_{j \nu}\left(f_{j \rho} \mathbf{u}_{\rho}\right)-D_{i \mu}^{\times} \tilde{D}_{\mu \nu}^{-1} D_{j \nu}^{\times}\left(f _ { j \rho } \left(\mathbf{u}\left(\mathbf{u}_{\mu}\left(\mu^{\prime}\right)\right)\right.\right. \\
& \left.=N_{i \mu} D_{\mu \nu} P_{\nu \rho}^{C G} \mathbf{u}_{\rho}-D_{i \mu}^{\times} \tilde{D}_{\mu \nu}^{-1} P_{\nu \tau}^{\perp} D_{\tau \nu} P_{\nu \rho}^{C G} \mathbf{u}_{k} 45\right) \\
& =N_{i \mu} D_{\mu \nu} \mathbf{u}_{\nu}-D_{i \mu}^{\times} \tilde{D}_{\mu \nu}^{-1} P_{\nu \tau}^{\perp} D_{\tau \nu} \mathbf{u}_{\nu} \\
& =0
\end{aligned}
$$

Thus the atoms (and nodes) of the coarse-grained system experience no force. In going from the second to the third line we have used the admissibility condition that $P_{\nu \rho}^{C G}$ act as the identity matrix on $\mathbf{u}_{\rho}$, and in the following line we used that $\mathbf{u}_{\nu}$ is in equilibrium and so $D_{\tau \nu} \mathbf{u}_{\nu}=0$. This derivation proves that any admissible equilibrium atomistic configuration is also an equilibrium CGMD configuration. The derivation continues to work once anharmonic forces are included, as discussed below, since the terms in the CGMD energy up to second order in displacements are the same. Thus, CGMD is free of ghost forces in both senses of the term.

\section{Anharmonic Lattices}

We have formulated CGMD for an underlying anharmonic Hamiltonian in perturbation theory, assuming again negligible diffusion in the $\mathrm{CG}$ region. With an anharmonic potential the higher frequency modes comprising the heat bath do not decouple, and they introduce temperature-dependent effects such as thermal expansion and thermal softening of the elastic constants. The basic 
idea of how anharmonicity is treated was presented in Ref. 3. The details are presented here for the first time.

In this subsection we develop a formal analysis of the contributions of the anharmonic interatomic forces to the CGMD energy and equations of motion. This analysis provides insight into how effects in the coarse-grained system are linked to their atomistic origins through analytic formulas, and hence are very powerful. On the other hand, the formulas are sufficiently complicated that a different approach is employed in practice, and we stress this point. The formal developments that follow take the perfect $T=0 \mathrm{~K}$ lattice as the reference state; in practice, it is much more useful to take the lattice at the temperature of interest to be the reference state. In that case, the harmonic theory presented above may be used, with anharmonic effects from the lattice entering into the reference state in a quasi-harmonic approach. The thermal expansion of the lattice and the thermal softening of the dynamical matrix capture the anharmonic forces ${ }^{17}$ The finite temperature lattice constant and dynamical matrix are inputs to the CGMD formulation, precomputed in conventional MD calculations. While that approach is very effective in practice, it is more satisfying from a theoretical point of view to have a direct, analytic theory of the thermal effects in CGMD, and it is to this development we turn now.

The CGMD energy (16) is computed by perturbation theory about the harmonic Hamiltonian (15). Specifically,

$$
H_{M D}=H_{h}+H^{\prime}
$$

where $H_{h}$ is the harmonic Hamiltonian (15) and $H^{\prime}$ consists of the anharmonic corrections, which are assumed to be small. This is a good approximation in silicon, and many other materials of interest below their melting point (i.e. at low homologous temperatures). The perturbation may be written explicitly as

$$
H^{\prime}=\sum_{n=3}^{\infty} \frac{1}{n !} \sum D_{\mu_{1} \ldots \mu_{n}}^{a_{1} \ldots a_{n}} u_{\mu_{1}}^{a_{1}} \cdots u_{\mu_{n}}^{a_{n}}
$$

where the $\mu$ 's label the atoms, as before, and the $a$ 's label components of the vectors. The higher order dynamical matrices $D_{\mu_{1} \ldots \mu_{n}}^{a_{1} \ldots a_{n}}$ may be taken to be completely symmetric in their indices from the form of Eq. (49). Below we occasionally use the notation $D^{(n)}$ to represent $D_{\mu_{1} \ldots \mu_{n}}^{a_{1} \ldots a_{n}}$ schematically in places where the additional concision should not lead to confusion. This perturbation theory is a low temperature expansion, as seen by switching to the scaled coordinates

$$
\begin{aligned}
\tilde{\mathbf{u}}_{\mu}=\frac{\mathbf{u}_{\mu}}{\sqrt{k T}}, & & \tilde{\mathbf{u}}_{j}=\frac{\mathbf{u}_{j}}{\sqrt{k T}}, \\
\tilde{\mathbf{p}}_{\mu}=\frac{\mathbf{p}_{\mu}}{\sqrt{k T}}, & & \dot{\tilde{\mathbf{u}}}_{j}=\frac{\dot{\mathbf{u}}_{j}}{\sqrt{k T}}, \\
\tilde{\lambda}_{j}=\sqrt{k T} \lambda_{j}, & & \tilde{\lambda}_{j}^{\prime}=\sqrt{k T} \lambda_{j} .
\end{aligned}
$$

Then

$$
\begin{aligned}
E & =U_{\mathrm{int}}^{h}-\partial_{\beta} \log \int d \tilde{\mathbf{u}}_{\mu} d \tilde{\mathbf{p}}_{\mu} e^{-\tilde{H}_{h}} e^{-H^{\prime}} \Delta, \\
\tilde{H}_{h} & =\sum_{\mu} \frac{\tilde{\mathbf{p}}_{\mu}^{2}}{2 m}+\sum_{\mu, \nu} \frac{1}{2} \tilde{\mathbf{u}}_{\mu} \cdot D_{\mu \nu} \tilde{\mathbf{u}}_{\nu}, \\
\tilde{H}^{\prime} & =\sum_{n=3}^{\infty} \frac{1}{n !} \sum \beta^{(2-n) / 2} D_{\mu_{1} \ldots \mu_{n}}^{a_{1} \ldots a_{n}} \tilde{u}_{\mu_{1}}^{a_{1}} \ldots \tilde{u}_{\mu_{n}}^{a_{n}}
\end{aligned}
$$

where $U_{\text {int }}^{h}$ is the internal energy for the CG harmonic lattice (29). The temperature dependence is now in $U_{\text {int }}^{h}$, $\Delta$, and in the perturbation $\tilde{H}^{\prime}$, but not in $H_{h}$.

To evaluate this integral, the exponential involving $\tilde{H}^{\prime}$ is expanded in a Taylor series in $\tilde{u}$ and/or $k T$. The resulting integrals are elementary. It is common practice to use a diagrammatic representation of the integrals to facilitate bookkeeping in this kind of expansion ${ }^{47}$ The $\log$ in Eq. (53) is then produced by restricting to connected graphs ${ }^{48}$ Similar perturbative approaches have been used in many contexts; the application most relevant to the current study is the Self-Consistent Phonon Approximation used in lattice dynamics ${ }^{49.50}$

The resulting form of the CG energy for the anharmonic lattice is

$$
\begin{array}{r}
E\left(\mathbf{u}_{k}, \dot{\mathbf{u}}_{k}\right)=U_{\mathrm{int}}+\sum_{j, k} \frac{1}{2}\left(M_{j k} \dot{\mathbf{u}}_{j} \cdot \dot{\mathbf{u}}_{k}+\mathbf{u}_{j} \cdot K_{j k} \mathbf{u}_{k}\right)+ \\
\sum_{n=1}^{\infty} \frac{1}{n !} \sum K_{j_{1} \ldots j_{n}}^{a_{1} \ldots a_{n}}(T) u_{j_{1}}^{a_{1}} \cdots u_{j_{n}}^{a_{n}}(56)
\end{array}
$$

where now $U_{\text {int }}$ is a complicated function of $\beta$, as are the stiffness coefficients. Our goal is to calculate this expansion analytically at each order of perturbation theory. Since the diagrammatic approach may not be familiar to all readers, we will not use it for the derivations, but we return to it below. For now, consider the integral needed to calculate the CGMD energy up to second order in $D^{(3)}$ and first order in $D^{(4)}$. These first few terms in the perturbation series will capture the leading thermal and non-linear effects. Higher order terms could be calculated similarly, if needed. To this order, the CGMD potential energy expansion is

$$
\begin{aligned}
U= & U_{\mathrm{int}}^{h}-\partial_{\beta} \log \int d \tilde{\mathbf{u}}_{\mu} d \tilde{\mathbf{p}}_{\mu}[1 \\
& -\frac{\beta^{-1 / 2}}{3 !} D_{\mu_{1} \mu_{2} \mu_{3}}^{a_{1} a_{2} a_{3}} \tilde{u}_{\mu_{1}}^{a_{1}} \tilde{u}_{\mu_{2}}^{a_{2}} \tilde{u}_{\mu_{3}}^{a_{3}} \\
& +\frac{1}{2} \beta^{-1}\left(\frac{1}{3 !} D_{\mu_{1} \mu_{2} \mu_{3}}^{a_{1} a_{2} a_{3}} \tilde{u}_{\mu_{1}}^{a_{1}} \tilde{u}_{\mu_{2}}^{a_{2}} \tilde{u}_{\mu_{3}}^{a_{3}}\right)^{2} \\
& \left.-\frac{1}{4 !} \beta^{-1} D_{\mu_{1} \mu_{2} \mu_{3} \mu_{4}}^{a_{1} a_{2} a_{3} a_{4}} \tilde{u}_{\mu_{1}}^{a_{1}} \tilde{u}_{\mu_{2}}^{a_{2}} \tilde{u}_{\mu_{3}}^{a_{3}} \tilde{u}_{\mu_{4}}^{a_{4}}+\cdots\right] e^{-\tilde{H}_{0}} \Delta,
\end{aligned}
$$

where the polynomial terms have resulted from expanding the anharmonic exponential (55) in a Taylor series. In classic lattice dynamics analysis, a similar expansion 
is used. There the cubic term is odd in $\tilde{u}$ and integrates to zero, while the other two terms give non-zero contributions to the heat capacity of the order $k T{ }^{50}$ In CGMD, each of the three terms gives a non-zero contribution, as we will now show.

Integrals of the form (57), a polynomial times a Gaussian, may be evaluated in closed form. The algebra can be tedious, so often generating functions are used to simplify the bookkeeping. The CGMD generating function is defined to be

$$
Z_{h}[J]=Z_{\text {kin }} \int d \tilde{\mathbf{u}}_{\mu} e^{-\frac{1}{2} \tilde{\mathbf{u}}_{\mu} \cdot D_{\mu \nu} \tilde{\mathbf{u}}_{\nu}} e^{\tilde{\mathbf{J}}_{\mu} \cdot \tilde{\mathbf{u}}_{\mu}} \Delta
$$

where $Z_{\text {kin }}$ is the part of the partition function coming from the kinetic energy and including the nodal velocity constraints. The subscript on $Z_{h}$ indicates that it is the generating function corresponding to the harmonic theory. Generating functions are useful because derivatives with respect to $\tilde{J}_{\mu}$ bring down factors of $\tilde{\mathbf{u}}_{\mu}$,

$$
\left.\partial_{\tilde{\mathbf{J}}_{\lambda}} Z_{h}[J]\right|_{J=0}=Z_{\text {kin }} \int d \tilde{\mathbf{u}}_{\mu} e^{-\frac{1}{2} \tilde{\mathbf{u}}_{\mu} \cdot D_{\mu \nu} \tilde{\mathbf{u}}_{\nu}} \tilde{\mathbf{u}}_{\lambda} \Delta
$$

so they provide a simple way of calculating the integrals of Gaussians multiplied by a polynomial prefactor. The CGMD internal energy (57) may therefore be computed from the generating function as

$$
U=U_{\text {int }}^{h}-\partial_{\beta} \log \left\{\left.\exp \left[-\beta H^{\prime}\left(\partial_{\tilde{\mathbf{J}}}\right)\right] Z_{h}[J]\right|_{J=0}\right\}
$$

where the factor $\exp \left[-\beta H^{\prime}\left(\partial_{\tilde{J}}\right)\right]$ is intended to be expanded in its Taylor series for practical calculations.

We return to the anharmonic terms below [cf. Eq. (71)], but first we compute the partition function. In order to compute $Z_{h}[J]$ we can complete the square and relate the result to $Z_{h}[0]$ :

$$
\begin{aligned}
Z_{h}[J]= & Z_{\mathrm{kin}} e^{\frac{1}{2} \tilde{\mathbf{J}}_{\mu} \cdot D_{\mu \nu}^{-1} \tilde{\mathbf{J}}_{\nu}} \int d \tilde{\mathbf{w}}_{\mu} e^{-\frac{1}{2} \tilde{\mathbf{w}}_{\mu} \cdot D_{\mu \nu} \tilde{\mathbf{w}}_{\nu}} \times(61) \\
& \prod \delta\left(\mathbf{u}_{\mathbf{j}}-f_{j \mu} \tilde{\mathbf{w}}_{\mu}-f_{j \mu} D_{\mu \nu}^{-1} \cdot \tilde{\mathbf{J}}_{\nu}\right) \\
= & e^{\frac{1}{2} \tilde{\mathbf{J}}_{\mu} \cdot D_{\mu \nu}^{-1} \tilde{\mathbf{J}}_{\nu}} Z_{h}\left[J=0 ; \tilde{\mathbf{u}}_{j}-f_{j \mu} D_{\mu \nu}^{-1} \cdot \tilde{\mathbf{J}}_{\nu}\right](62) \\
= & Z_{h}[J=0] e^{-\frac{1}{2} \tilde{\mathbf{J}}_{\mu} \cdot \mathbf{G}_{\mu \nu} \cdot \tilde{\mathbf{J}}_{\nu}-\tilde{\mathbf{H}}_{\kappa} \cdot \tilde{\mathbf{J}}_{\kappa}}
\end{aligned}
$$

where $\tilde{\mathbf{w}}_{\mu}=\tilde{\mathbf{u}}_{\mu}-D_{\mu \nu}^{-1} \cdot \tilde{\mathbf{J}}_{\nu}$. We have used Eq. (29) to derive the third line. The Green function $\mathbf{G}_{\mu \nu}$ and the external field $\mathbf{H}_{\mu}$ are given by

$$
\begin{aligned}
\mathbf{G}_{\mu \nu} & =D_{\mu \kappa}^{-1} f_{j \kappa} K_{j k} f_{k \lambda} D_{\lambda \nu}^{-1}-D_{\mu \nu}^{-1} \\
\mathbf{H}_{\mu} & =\mathbf{u}_{j} K_{j k} f_{k \nu} D_{\nu \mu}^{-1}
\end{aligned}
$$

with $\tilde{\mathbf{H}}_{\mu}=\beta^{1 / 2} \mathbf{H}_{\mu}$.

Using the projection matrices defined in Appendix $\mathrm{A}$ we can rewrite this expression in a more transparent form. The projection matrices are given by $P_{\mu \nu}^{\mathrm{CG}}=$ $N_{j \mu} f_{j \nu}$ and $P_{\mu \nu}^{\perp}=\delta_{\mu \nu}-P_{\mu \nu}^{\mathrm{CG}}$. Using them together with the formula for $K_{i j}$ (36) we have the alternate formulas

$$
\begin{aligned}
\mathbf{G}_{\mu \nu} & =P_{\mu \rho}^{\perp}\left(\tilde{D}_{\rho \kappa}^{-1} f_{j \kappa} K_{j k} f_{k \lambda} \tilde{D}_{\lambda \sigma}^{-1}-\tilde{D}_{\rho \sigma}^{-1}\right) P_{\sigma \nu}^{\perp} \\
\mathbf{H}_{\mu} & =\mathbf{u}_{j} N_{j \mu}+\mathbf{u}_{j} K_{j k} f_{k \nu} \tilde{D}_{\nu \lambda}^{-1} P_{\lambda \mu}^{\perp}
\end{aligned}
$$

where $\tilde{D}_{\mu \nu}$ is defined by Eq. (A16) 51 These formulas show that in the rigid approximation $\mathbf{G}_{\mu \nu}=0$ and $\mathbf{H}_{\mu}=\mathbf{u}_{j} N_{j \mu}$. The result is that each occurrence of $\mathbf{u}_{\mu}$ in the MD potential energy is just replaced by $N_{j \mu} \mathbf{u}_{j}$ in R-CGMD. Relaxation of the unconstrained degrees of freedom makes the further contributions involving $P_{\mu \nu}^{\perp}$.

To be more explicit, the rigid approximation to CGMD freezes the unconstrained (unresolved) modes. Formally, this is accomplished by setting the orthogonal projectors to zero: $P_{\mu \nu}^{\perp} \rightarrow 0$. In this approximation the generating function simplifies

$$
\left.Z_{h}[J]\right|_{\text {rigid }}=Z_{h}[0] e^{-\beta^{1 / 2} \mu_{j} N_{j \mu} \tilde{J}_{\mu}}
$$

where all of the Green function contributions have been eliminated. Similarly, the mass and stiffness matrices in $Z_{h}[0]$ involve only the first terms in Eqs. (38) and (39). The Rigid CGMD (R-CGMD) Hamiltonian is then given by

$$
E\left(\mathbf{u}_{k}, \dot{\mathbf{u}}_{k}\right)=U_{\mathrm{int}}+\frac{1}{2} M_{i j} \dot{\mathbf{u}}_{i} \cdot \dot{\mathbf{u}}_{j}+U_{M D}\left(N_{j \mu} \mathbf{u}_{j}\right),
$$

where $U_{M D}$ is the full, non-linear MD potential energy evaluated with $\mathbf{u}_{\mu}=N_{j \mu} \mathbf{u}_{j}$, and $M_{i j}=N_{j \mu} m_{\mu} N_{k \mu}$. The rigid approximation neglects thermal effects and relaxation of the internal degrees of freedom (i.e., those that have been integrated out). This expression is a conserved energy, and it is free from spurious forces commonly referred to as 'ghost forces' in discussions of concurrent multiscale modeling ${ }^{28}$ It is also as computationally expensive as MD, because unlike the stiffness matrix, the non-linear potential cannot be pre-computed except perhaps for some toy potentials. Additional approximations are needed. One set of approximations based on the use of representative atoms and the Cauchy-Born rule leads to the Quasicontinuum Method ${ }^{24}$ Thus, the derivation above shows the relationship between CGMD and the Quasicontinuum Method, in particular that the Quasicontinuum Method is closely related to the zero temperature rigid approximation of CGMD. Another successful concurrent multiscale technique, Coupling of Length Scale (CLS) ${ }^{23}$, can also be related to CGMD. It involves taking the large- $N$ asymptotic limit of the stiffness matrix in the rigid approximation, where $N$ in this case is the number of atoms per element. The large- $N$ limit yields continuum mechanics within the element; i.e., a finite element representation of continuum mechanics. Both CLS and the Quasicontinuum Method make additional approximations at the MD/CG interface.

We now consider the physics of the terms in the CGMD energy beyond the rigid approximation. These are effects that arise due to the anharmonicity of the interatomic potential, and include both thermal effects and non-linear relaxation. The diagrammatic approach is a convenient and powerful way to analyze perturbation theory at higher order, such as the anharmonic contributions to CGMD. The quantities represented by the diagrams are typically generated from a relatively simple set of 


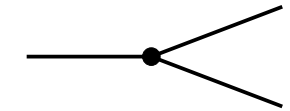

$\beta \mathrm{D}^{(3)} \mathrm{H}^{3}$

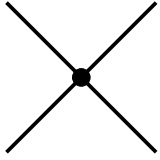

$\beta \mathrm{D}^{(4)} \mathrm{H}^{4}$

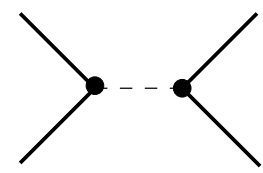

$\beta \mathrm{D}^{(3)} \mathrm{H}^{2} \mathrm{GD}^{(3)} \mathrm{H}^{2}$

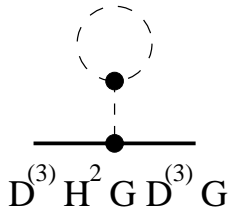

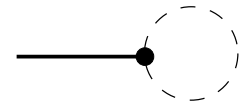

$\mathrm{D}^{(3)} \mathrm{H} \mathrm{G}$

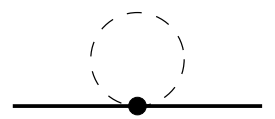

$\mathrm{D}^{(4)} \mathrm{G} \mathrm{H}^{2}$

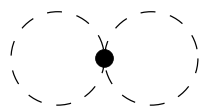

$\beta^{-1} D^{(4)} G^{2}$

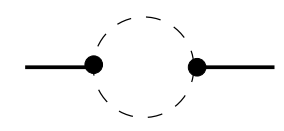

$\mathrm{D}^{(3)} \mathrm{H} \mathrm{G}^{2} \mathrm{D}^{(3)} \mathrm{H}$

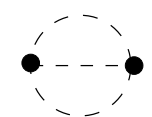

$\beta^{-1} D^{(3)} G^{3} D^{(3)}$
FIG. 2: Feynman diagrams of connected graphs contributing to the anharmonic CGMD internal energy. The thick external legs represent factors of $H$, which in the usual terminology correspond to dressed external fields. Physically, they are contributions from the CG fields with some zero temperature relaxation of the internal degrees of freedom. The dashed internal lines represent factors of $G$ that arise from the fluctuations of the internal degrees of freedom. These fluctuations are temperature dependent, and each factor of $G$ comes with a factor of $\beta^{-1}(=k T)$.

rules, known as Feynman rules. Due to space limitations we cannot provide a thorough review of the rich mathematical structure encoded in Feynman diagrams, but direct the interested reader to one of the numerous texts on the subject. 48 We have derived the Feynman rules for CGMD, but they will not be presented here since they are not actually needed. Instead, we will employ the set of Feynman diagrams as a pedagogical device to consider the form of various contributions to the CGMD energy. The Feynman diagrams up to second order in $D^{(3)}$ and first order in $D^{(4)}$ are shown in Fig. 2 In the diagrams, the thick external legs represent factors of $\mathbf{H}_{\mu}$; i.e., factors of the CG fields $\mathbf{u}_{j}$, suitably dressed to account for elastic relaxation of the internal degrees of freedom. These factors are playing the role of external fields in the statistical field theory of the equilibrium state of the internal degrees of freedom. The thinner, dashed lines represent factors of the Green function $\mathbf{G}_{\mu \nu}$. The Green function accounts for thermal fluctuations of the internal degrees of freedom, and indeed each factor of $\mathbf{G}_{\mu \nu}$ brings with it a factor of $k T$. Vertices in the graphs denote contracted tensor indices.

At a given level of perturbation theory, the graphs pictorially represent the hierarchy of contributions to the CGMD energy. Although graphs that split into more than one disconnected sub-graph contribute to the partition function, only connected graphs contribute to the free energy 48 These are shown in Fig. 2 Consider first the graphs with no external legs. These terms in the perturbation theory are independent of $\mathbf{u}_{j}$; they only contribute to the internal energy resulting from degrees of freedom that have been integrated out. Thus they build up the non-trivial temperature dependence of $U_{\text {int }}$. The graphs at the other extreme-in particular those that have no dashed lines and are therefore independent of $\mathbf{G}_{\mu \nu}$-make up the rigid approximation that was discussed above, but now including some internal relaxation.

Using the generating function (63), we have calculated the CGMD internal energy (57) up to first order in $D^{(4)}$ and second order in $D^{(3)}$ :

$$
\begin{aligned}
U= & U_{\text {int }}^{h}-\partial_{\beta} \log Z \\
Z= & \left(1-\frac{\beta^{-1 / 2}}{6} D_{\mu_{1} \mu_{2} \mu_{3}}^{a_{1} a_{2} a_{3}} \partial_{\tilde{J}_{\mu_{1}}^{a_{1}}} \partial_{\tilde{J}_{\mu_{2}}^{a_{3}}} \partial_{\tilde{J}_{\mu_{3}}^{a_{3}}}\right. \\
& +\frac{\beta^{-1}}{72} D_{\mu_{1} \mu_{2} \mu_{3}}^{a_{1} a_{2} a_{3}} D_{\mu_{4} \mu_{5} \mu_{6}}^{a_{4} a_{5} a_{6}} \partial_{\tilde{J}_{\mu_{1}}^{a_{1}}} \partial_{\tilde{J}_{\mu_{2}}^{a_{3}}} \partial_{\tilde{J}_{\mu_{3}}^{a_{3}}} \partial_{\tilde{J}_{\mu_{4}}^{a_{4}}} \partial_{\tilde{J}_{\mu_{5}}^{a_{5}}} \partial_{\tilde{J}_{\mu_{6}}^{a_{6}}} \\
& \left.\quad-\frac{\beta^{-1}}{24} D_{\mu_{1} \mu_{2} \mu_{3} \mu_{4}}^{a_{1} a_{2} a_{3} a_{4}} \partial_{\tilde{J}_{\mu_{1}}^{a_{1}}} \partial_{\tilde{J}_{\mu_{2}}^{a_{3}}} \partial_{\tilde{J}_{\mu_{3}}^{a_{3}}} \partial_{\tilde{J}_{\mu_{4}}^{a_{4}}}+\ldots\right)\left.Z_{h}[\tilde{J}]\right|_{\tilde{J}=0}
\end{aligned}
$$

where $Z_{h}[\tilde{J}]$ is given by Eq. (63). The derivatives act on the Gaussian generating function (63) to give

$$
\begin{aligned}
& Z=\left[1+\frac{1}{2} D_{\mu_{1} \mu_{2} \mu_{3}}^{a_{1} a_{2} a_{3}} G_{\mu_{1} \mu_{2}}^{a_{1} a_{2}} H_{\mu_{3}}^{a_{3}}\right. \\
& +\frac{1}{2}\left(\frac{1}{2} D_{\mu_{1} \mu_{2} \mu_{3}}^{a_{1} a_{2} a_{3}} G_{\mu_{1} \mu_{2}}^{a_{1} a_{2}} H_{\mu_{3}}^{a_{3}}\right)^{2} \\
& +\frac{\beta}{8} D_{\mu_{1} \mu_{2} \mu_{3}}^{a_{1} a_{2} a_{3}} D_{\mu_{4} \mu_{5} \mu_{6}}^{a_{4} a_{5} a_{6}} G_{\mu_{1} \mu_{4}}^{a_{1} a_{4}} H_{\mu_{2}}^{a_{2}} H_{\mu_{3}}^{a_{3}} H_{\mu_{5}}^{a_{5}} H_{\mu_{6}}^{a_{6}} \\
& +\frac{1}{4} D_{\mu_{1} \mu_{2} \mu_{3}}^{a_{1} a_{2} a_{3}} D_{\mu_{4} \mu_{5} \mu_{6}}^{a_{4} a_{5} a_{6}} G_{\mu_{1} \mu_{4}}^{a_{1} a_{4}} G_{\mu_{2} \mu_{5}}^{a_{2} a_{5}} H_{\mu_{3}}^{a_{3}} H_{\mu_{6}}^{a_{6}} \\
& -\frac{\beta^{-1}}{12} D_{\mu_{1} \mu_{2} \mu_{3}}^{a_{1} a_{2} a_{3}} D_{\mu_{4} \mu_{5} \mu_{6}}^{a_{4} a_{5} a_{6}} G_{\mu_{1} \mu_{4}}^{a_{1} a_{4}} G_{\mu_{2} \mu_{5}}^{a_{2} a_{5}} G_{\mu_{3} \mu_{6}}^{a_{3} a_{6}} \\
& +\frac{1}{4} D_{\mu_{1} \mu_{2} \mu_{3}}^{a_{1} a_{2} a_{3}} D_{\mu_{4} \mu_{5} \mu_{6}}^{a_{4} a_{5} a_{6}} G_{\mu_{1} \mu_{4}}^{a_{1} a_{4}} G_{\mu_{2} \mu_{3}}^{a_{2} a_{3}} H_{\mu_{5}}^{a_{5}} H_{\mu_{6}}^{a_{6}} \\
& -\frac{\beta^{-1}}{8} D_{\mu_{1} \mu_{2} \mu_{3}}^{a_{1} a_{2} a_{3}} D_{\mu_{4} \mu_{5} \mu_{6}}^{a_{4} a_{5} a_{6}} G_{\mu_{1} \mu_{4}}^{a_{1} a_{4}} G_{\mu_{2} \mu_{3}}^{a_{2} a_{3}} G_{\mu_{5} \mu_{6}}^{a_{5} a_{6}} \\
& +D_{\mu_{1} \mu_{2} \mu_{3} \mu_{4}}^{a_{1} a_{2} a_{3} a_{4}} \times \\
& \left(-\frac{1}{4} G_{\mu_{1} \mu_{2}}^{a_{1} a_{2}} H_{\mu_{3}}^{a_{3}} H_{\mu_{4}}^{a_{4}}+\frac{\beta^{-1}}{8} G_{\mu_{1} \mu_{2}}^{a_{1} a_{2}} G_{\mu_{3} \mu_{4}}^{a_{3} a_{4}}\right) \\
& +\ldots] e^{-\beta H^{\prime}\left(\mathbf{u}_{\mu}=\mathbf{H}_{\mu}\right)} Z_{h}[0]
\end{aligned}
$$


Next we find the Helmholtz free energy $F=-k T \log Z$ :

$$
\begin{aligned}
& F=F_{\text {harmonic }}+H^{\prime}\left(\mathbf{u}_{\mu}=\mathbf{H}_{\mu}\right) \\
&-\frac{1}{8} D_{\mu_{1} \mu_{2} \mu_{3}}^{a_{1} a_{2} a_{3}} D_{\mu_{4} \mu_{5} \mu_{6}}^{a_{4} a_{5} a_{6}} G_{\mu_{1} \mu_{4}}^{a_{1} a_{4}} H_{\mu_{2}}^{a_{2}} H_{\mu_{3}}^{a_{3}} H_{\mu_{5}}^{a_{5}} H_{\mu_{6}}^{a_{6}} \\
&-\frac{1}{2} k T D_{\mu_{1} \mu_{2} \mu_{3}}^{a_{1} a_{2} a_{3}} G_{\mu_{1} \mu_{2}}^{a_{1} a_{2}} H_{\mu_{3}}^{a_{3}} \\
& \quad-\frac{1}{4} k T D_{\mu_{1} \mu_{2} \mu_{3}}^{a_{1} a_{2} a_{3}} D_{\mu_{4} \mu_{5} \mu_{6}}^{a_{4} a_{5} a_{6}} G_{\mu_{1} \mu_{4}}^{a_{1} a_{4}} G_{\mu_{2} \mu_{5}}^{a_{2} a_{5}} H_{\mu_{3}}^{a_{3}} H_{\mu_{6}}^{a_{6}} \\
&-\frac{1}{4} k T D_{\mu_{1} \mu_{2} \mu_{3}}^{a_{1} a_{2} a_{3}} D_{\mu_{4} \mu_{5} \mu_{6}}^{a_{4} a_{5} a_{6}} G_{\mu_{1} \mu_{4}}^{a_{1} a_{4}} G_{\mu_{2} \mu_{3}}^{a_{2} a_{3}} H_{\mu_{5}}^{a_{5}} H_{\mu_{6}}^{a_{6}} \\
&+\frac{1}{4} k T D_{\mu_{1} \mu_{2} \mu_{3} \mu_{4}}^{a_{1} a_{2} a_{3} a_{4}} G_{\mu_{1} \mu_{2}}^{a_{1} a_{2}} H_{\mu_{3}}^{a_{3}} H_{\mu_{4}}^{a_{4}} \\
&-\frac{1}{8}(k T)^{2} D_{\mu_{1} \mu_{2} \mu_{3} \mu_{4}}^{a_{1} a_{2} a_{3} a_{4}} G_{\mu_{1} \mu_{2}}^{a_{1} a_{2}} G_{\mu_{3} \mu_{4}}^{a_{3} a_{4}} \\
&+\frac{1}{12}(k T)^{2} D_{\mu_{1} \mu_{2} \mu_{3}}^{a_{1} a_{2} a_{3}} D_{\mu_{4} \mu_{5} \mu_{6}}^{a_{4} a_{5} a_{6}} G_{\mu_{1} \mu_{4}}^{a_{1} a_{4}} G_{\mu_{2} \mu_{5}}^{a_{2} a_{5}} G_{\mu_{3} \mu_{6}}^{a_{3} a_{6}} \\
&+\frac{1}{8}(k T)^{2} D_{\mu_{1} \mu_{2} \mu_{3}}^{a_{1} a_{2} a_{3}} D_{\mu_{4} \mu_{5} \mu_{6}}^{a_{4} a_{5} a_{6}} G_{\mu_{1} \mu_{4}}^{a_{1} a_{4}} G_{\mu_{2} \mu_{3}}^{a_{2} a_{3}} G_{\mu_{5} \mu_{6}}^{a_{5} a_{6}} \\
&+\ldots
\end{aligned}
$$

where $F_{\text {harmonic }}$ is the CGMD free energy for a harmonic crystal [cf. Eq. (29)]:

$$
\begin{aligned}
F_{\text {harmonic }} & =F_{\text {int }}+\frac{1}{2} \sum_{j, k}\left(M_{j k} \dot{\mathbf{u}}_{j} \cdot \dot{\mathbf{u}}_{k}+\mathbf{u}_{j} \cdot K_{j k} \mathbf{u}_{k}\right)(74) \\
F_{\text {int }} & \left.=N_{\text {atom }} E^{\text {coh }}-3\left(N_{\text {atom }}-N_{\text {node }}\right) k T \log (k 75)\right)
\end{aligned}
$$

The free energy is suitable for isothermal CGMD simulations. On the other hand, the internal energy is appropriate for adiabatic simulations, and hence more closely related to MD simulations without a thermostat. It is given by $U=\partial_{\beta}(\beta F)$ :

$$
\begin{aligned}
& U=E_{\text {harmonic }}+H^{\prime}\left(\mathbf{u}_{\mu}=\mathbf{H}_{\mu}\right) \\
& \quad-\frac{1}{8} D_{\mu_{1} \mu_{2} \mu_{3}}^{a_{1} a_{2} a_{3}} D_{\mu_{4} \mu_{5} \mu_{6}}^{a_{4} a_{5} a_{6}} G_{\mu_{1} \mu_{4}}^{a_{1} a_{4}} H_{\mu_{2}}^{a_{2}} H_{\mu_{3}}^{a_{3}} H_{\mu_{5}}^{a_{5}} H_{\mu_{6}}^{a_{6}} \\
& \quad+\frac{1}{8}(k T)^{2} D_{\mu_{1} \mu_{2} \mu_{3} \mu_{4}}^{a_{1} a_{2} a_{3} a_{4}} G_{\mu_{1} \mu_{2}}^{a_{1} a_{2}} G_{\mu_{3} \mu_{4}}^{a_{3} a_{4}} \\
& \quad-\frac{1}{12}(k T)^{2} D_{\mu_{1} \mu_{2} \mu_{3}}^{a_{1} a_{2} a_{3}} D_{\mu_{4} \mu_{5} \mu_{6}}^{a_{4} a_{5} a_{6}} G_{\mu_{1} \mu_{4}}^{a_{1} a_{4}} G_{\mu_{2} \mu_{5}}^{a_{2} a_{5}} G_{\mu_{3} \mu_{6}}^{a_{3} a_{6}} \\
&-\frac{1}{8}(k T)^{2} D_{\mu_{1} \mu_{2} \mu_{3}}^{a_{1} a_{2} a_{3}} D_{\mu_{4} \mu_{5} \mu_{6}}^{a_{4} a_{5} a_{6}} G_{\mu_{1} \mu_{4}}^{a_{1} a_{4}} G_{\mu_{2} \mu_{3}}^{a_{2} a_{3}} G_{\mu_{5} \mu_{6}}^{a_{5} a_{6}} \\
&+\ldots
\end{aligned}
$$

where $E_{\text {harmonic }}$ is given by Eq. (29). Now $T$ is understood to be a function of the (constant) entropy and the state of deformation, although for many applications since the deformation in the CG region is small, it can be treated as constant. For reference, the expressions for the Green function $G_{\mu_{1} \mu_{2}}^{a_{1} a_{2}}$ and the field $H_{\mu}^{a}$ are given in Eqs. (66) and (67), respectively.

We could continue to calculate the terms in the CGMD Hamiltonian to higher order in the MD anharmonic corrections and/or the CGMD thermal perturbation expansion. The number of terms grows rapidly, and we quickly reach the point of marginal returns; i.e., the point at which the added complexity is no longer rewarded with a commensurate improvement in accuracy. However, there are certain kinds of contributions where improvements are possible. In particular, it is important to capture the first non-trivial effects in the expansion. The terms calculated thus far have contributed to the harmonic Hamiltonian, the zero-temperature anharmonic terms and the energy of the internal modes. We have also calculated the leading contributions in the free energy to the thermal expansion and temperature-dependence of the stiffness, i.e., the terms proportional to $\mathbf{u}_{j}$ and $\mathbf{u}_{j_{1}} \otimes \mathbf{u}_{j_{2}}$. In the internal energy, these contributions are higher order in the anharmonic lattice expansion, so we have not calculated them yet. Of course, there are thermal corrections to the higher order stiffnesses, as well, but they are not as important.

We conclude this section with the calculation of the leading temperature-dependent, quasi-harmonic contributions to the CGMD Hamiltonian (internal energy). The graphs contributing the leading temperature dependence to the one-point function (governing thermal expansion) are shown in Fig. [3] The term "one-point" means a single leg, and hence a single factor of $\mathbf{u}_{j}$. Consider the lowest order term in $k T$ in Fig. 3 as it enters the free energy:

$$
-\frac{1}{2} k T D_{\mu_{1} \mu_{2} \mu_{3}}^{a_{1} a_{2} a_{3}} G_{\mu_{1} \mu_{2}}^{a_{1} a_{2}} H_{\mu_{3}}^{a_{3}}=-\mathbf{f}_{j}^{0} \mathbf{u}_{j}
$$

where we define the temperature-dependent $\mathbf{f}_{j}^{0}$ (not to be confused with $f_{j \nu}$ ) to be:

$$
\left(\mathbf{f}_{j}^{0}\right)_{a}=-\frac{1}{2} k T D_{\mu_{1} \mu_{2} \mu_{3}}^{a_{1} a_{2} a} G_{\mu_{1} \mu_{2}}^{a_{1} a_{2}} f_{k \nu} D_{\nu \mu_{3}}^{-1} K_{j k}
$$

where we have used the expression (65) for $H_{\mu_{3}}^{a_{3}}$. We have written the leading one-point term from the free energy in Eq. (77) in a form familiar from finite element models as an eigenstrain (cf. Section 2.12 of Hughes ${ }^{21}$ ). As in conventional finite element formulations in uniform temperature, this term is a total difference (the discrete analog of a total derivative), and it only enters through the boundaries of the simulation. In a homogeneous system with free surfaces, the result is expansion of the system as the temperature increases. The expansion is proportional to $k T$ and $D^{(3)}$ to leading order, as expected based on conventional lattice dynamics. ${ }^{50}$ Note that since the linear term is a total difference, the discussion of ghost forces given above continues to be valid in the case of thermal expansion.

The graphs contributing the leading temperature dependence to the two-point function governing the temperature dependence of the adiabatic elastic stiffness are shown in Fig. 4. The leading contributions to the isothermal stiffness are the three graphs containing $G_{1}$ but with $G_{1}$ replaced by $G$. As expected, the first thermal contributions to the adiabatic stiffness are of order $(k T)^{2}$ due to the third law of thermodynamics (the Nernst theorem), whereas the first thermal contributions to the isothermal stiffness are of order $k T$. 


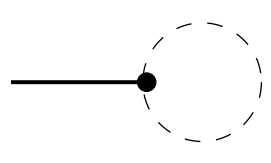

$D^{(3)} \mathrm{GH}$

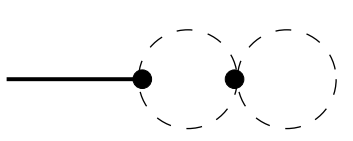

$\mathrm{kT} \mathrm{D}{ }^{(3)} \mathrm{H} \mathrm{G}^{2} \mathrm{D}^{(4)} \mathrm{G}$
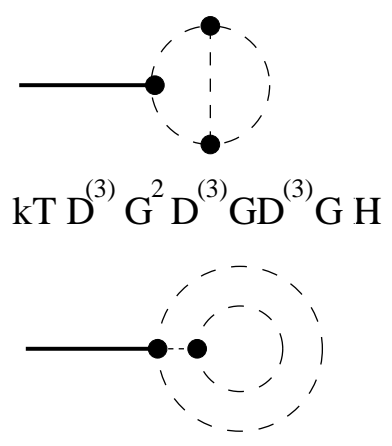

kT $D^{(4)} \mathrm{G}^{2} \mathrm{D}^{(3)} \mathrm{GH}$
FIG. 3: The leading anharmonic contributions to the partition function affecting the thermal expansion in CGMD.

Finally, we reemphasize that we have calculated properties within the CGMD perturbation theory to show that the theory is consistent and to gain some theoretical understanding of the interplay of anharmonicity and temperature in coarse-grained systems. In practice, we take the reference configuration to be the crystal lattice at a particular temperature with the corresponding finite temperature dynamical matrix. It would be a tautology to compute thermal expansion or thermal softening in CGMD: they agree with the MD result identically by construction.

\section{THERMAL FLUCTUATIONS}

In the previous Section we developed a description of the average motion of the collective degrees of freedom. We have shown that the short wavelength modes contribute to the mean motion, even though they are not explicitly present. They have the additional effect of inducing small fluctuations about this mean. The CG modes behave as if they were in a Brownian heat bath; they are jostled by small, random interactions with the (invisible, missing) short wavelength modes. In this section, we analyze these interactions in detail.

Consider the fluctuations of the CG fields in harmonic $\mathrm{MD}$ in thermal and mechanical equilibrium,

$$
\begin{aligned}
\left\langle\mathbf{u}_{j} \mathbf{u}_{k}\right\rangle & =f_{j \mu} f_{k \nu} Z^{-1} \int d \mathbf{x}_{\mu} d \mathbf{p}_{\mu} e^{-\beta H_{M D}} \mathbf{u}_{\mu} \mathbf{u}_{\nu} \\
& =f_{j \mu} f_{k \nu} k T \partial_{D_{\mu \nu}} \operatorname{tr} \log (D) \\
& =k T f_{j \mu} D_{\mu \nu}^{-1} f_{k \nu} \\
& =k T K_{j k}^{-1}
\end{aligned}
$$

This calculation shows that the two point isothermal correlation function of the $\mathrm{CG}$ displacements grows linearly with the temperature. The result is reasonable and could equally well be understood in terms of the equipartition theorem. As the temperature increases, the average potential energy increases and so the mean amplitude of the

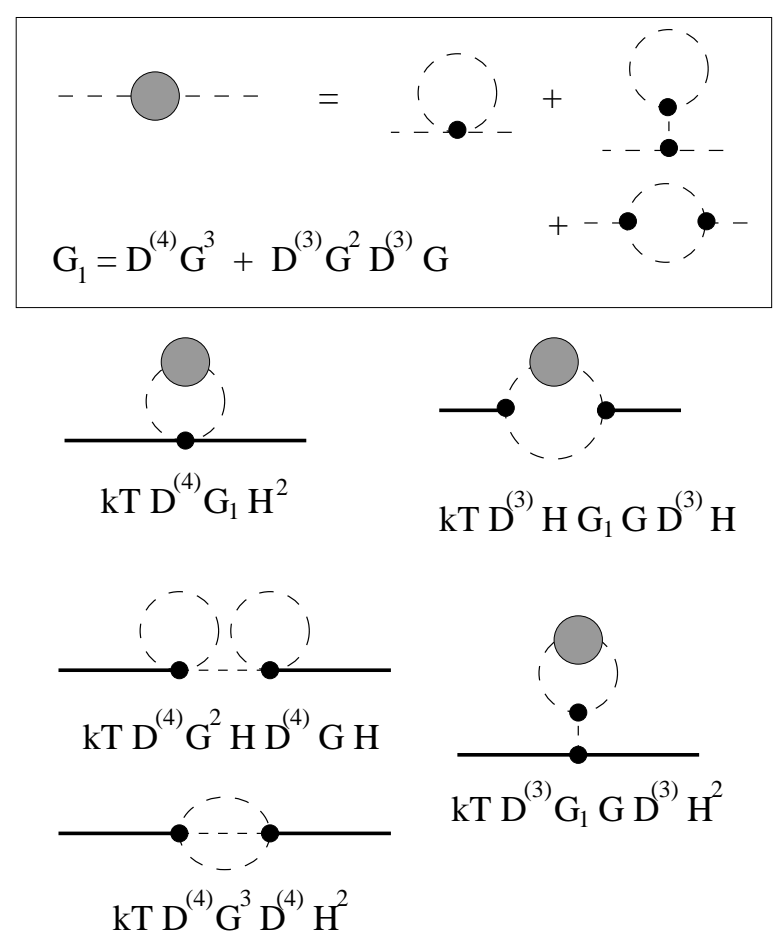

FIG. 4: The anharmonic contributions to the partition function affecting the temperature dependence of the adiabatic CGMD stiffness. The Green function $G_{1}$ is represented by a dashed line with a large filled circle. It is defined in the upper box.

harmonic oscillations increases. Note that the amplitude decreases as the stiffness $K_{j k}$ increases.

We can repeat the calculation in harmonic CGMD again in thermal equilibrium for comparison:

$$
\begin{aligned}
\left\langle\mathbf{u}_{j} \mathbf{u}_{k}\right\rangle_{T} & =Z^{-1} \int d \mathbf{u}_{j} d \dot{\mathbf{u}}_{j} e^{-\beta H_{C G M D}} \mathbf{u}_{j} \mathbf{u}_{k} \\
& =k T K_{j k}^{-1}
\end{aligned}
$$

where $H_{C G M D}$ is given by Eq. (29). The result is exactly the same as the correlation function of the $\mathrm{CG}$ fields in MD. This equivalence of the correlation functions holds for higher correlation functions as well, due to Wick's theorem. 52

While the equilibrium properties agree, there are differences in the time autocorrelation functions of the displacement. These differences result from the influence of the short wavelength modes that are not represented on the mesh. These additional modes acts as a heat bath, exerting random and dissipative forces on the CG fields. The effect is entirely analogous to Brownian motion, in which a large particle is jostled by the thermal motion of the unseen atoms in a liquid surrounding it.53

There are several practical issues that arise in the analysis of CGMD simulations regarding fluctuations. In conventional $\mathrm{MD}$, the mean atomic kinetic energy is used to compute the temperature. We now investigate whether 
a similar connection holds in the CG region of CGMD (naturally it continues to hold in the MD region if it is in thermal equilibrium). We consider the simplified case in which there is no large-scale motion such as center-ofmass motion; our derivation continues to hold provided any such non-equilibrated modes are subtracted from the nodal velocities prior to analysis. Consider the meansquared velocity of node $i$ in thermal equilibrium. We use the partition function for CGMD to calculate the expectation value of $\left|\dot{u}_{i}\right|^{2}$ in the canonical ensemble at thermal equilibrium:

$$
\begin{aligned}
\left\langle\left|\dot{u}_{i}\right|^{2}\right\rangle & =Z_{\text {kin }}^{-1} \int d \dot{u}\left|\dot{u}_{i}^{2}\right| e^{-\frac{1}{2} \beta M_{j k} \dot{\mathbf{u}}_{j} \cdot \dot{\mathbf{u}}_{k}} \\
& =\left.Z_{\text {kin }}^{-1} \partial_{J_{i}}^{2} \int d \dot{u} e^{-\frac{1}{2} \beta M_{j k} \dot{\mathbf{u}}_{j} \cdot \dot{\mathbf{u}}_{k}-\dot{\mathbf{J}}_{j} \cdot \dot{\mathbf{u}}_{j}}\right|_{J=0} \\
& =\left.\partial_{J_{i}}^{2} e^{\frac{1}{2} \beta^{-1} M_{j k}^{-1} \dot{\mathbf{J}}_{j} \cdot \dot{\mathbf{J}}_{k}}\right|_{J=0} \\
& =3 k T M_{i i}^{-1}
\end{aligned}
$$

where the factor of 3 in the final line is the number of dimensions. The potential energy contribution to the partition function is irrelevant to this calculation and has been suppressed. The important result expressed in Eq. (88) is that the mean-squared velocity is directly proportional to the temperature, just as it is in conventional MD.

In fact, the MD result is recovered by replacing $M_{i i}$ with the mass of atom $i$. This calculation also applies to concurrent multiscale models that use a lumped mass matrix. For CGMD with a non-diagonal mass matrix, it is only the diagonal of the mass matrix that affects the amplitude of oscillation of a given node. The off-diagonal terms do introduce correlations between the velocities of neighboring nodes that would not be present for a diagonal mass matrix. Because of these correlations, the simple atomistic relationship between the mean-squared velocity and the temperature that follows directly from the equipartition theorem must be modified according to Eq. (88) for use in CGMD.

Thus far in this article we have treated CGMD as a system that conserves energy, and these random, dissipative forces are absent. In particular, the evolution of the system generated by the Hamiltonian (3) conserves the energy given by that Hamiltonian. Thermostats might be added to simulate the electron-phonon coupling; i.e., the interaction of the lattice vibrations with the electronic degrees of freedom. Such additions violate energy conservation,,$\frac{54}{4}$ since energy can flow to and from the heat bath and the system becomes an open system.

Even neglecting the electron-phonon coupling, the coarse-grained system of solid mechanics described by CGMD is an open system. In the full MD system energy can flow between modes that would be retained in CGMD and those that would be integrated out $\frac{3}{\underline{3}}$ This interaction implies that the CGMD energy is conserved only on average, and that additional interactions are present in reality. These additional interactions take the form of random and dissipative forces. The form of these generalized Langevin forces may be computed using statistical mechanical (Zwanzig-Mori) projection operator techniques,$\frac{55}{5}$ although it is beyond the scope of this article. The resulting spatio-temporal memory kernel has been described elsewhere ${ }^{53}$ The random, dissipative forces not only act to bring the CG degrees of freedom into equilibrium with the internal degrees of freedom, but they act to absorb short wavelength modes incident on an interface where the mesh is refined. In principle, they also include the propagators that reconstruct waves on the far side of the CG region if the mesh is refined again; in practice, these propagators appear to be very expensive to implement computationally. In fact, one of the challenges of memory kernels is their computational expense both in terms of the memory required to store the recent history and in terms of the demands they place on parallelization to make the code suitable for supercomputers.

\section{IMPLEMENTATION DETAILS}

In practice, CGMD is run much the way conventional $\mathrm{MD}$ would be. The forces in the CG region are determined by the CGMD stiffness matrix and the nodal displacements; the forces in the MD region could be determined this way, as well, but since we have shown that the forces in the MD region are just the usual MD forces, the full MD potential is used to calculate the MD forces. Using the accelerations, the velocity Verlet time integrator is used to evolve the system in time ${ }^{56}$ The same time step is used throughout the simulation. In principle, the natural frequency in the CG region is lower as the mesh size increases, and a longer time step could be used there; in practice, the CG region entails relatively little computational expense, and there is little motivation to introduce a spatially varying time step that could cause subtle problems.

One difference from MD and conventional FEM is that the topology of the CG mesh is not allowed to change. Neighboring nodes remain neighboring nodes throughout the simulation. The topology of the mesh is determined by a cell list, which contains the nodes associated with each cell in the mesh, and a face list, which lists pairs of neighboring cells. An edge list, which lists pairs of neighboring nodes, is also generated.

The stiffness matrix $K_{i j}$ is to be computed once at the start of a simulation, and it remains unaltered during the subsequent dynamics. It does not matter whether atoms vibrate across cell boundaries, as long as the crystal lattice topology does not change and diffusion is negligible. The elements of $K_{i j}$ decrease exponentially with distance from the diagonal, and in practice it is necessary to truncate the stiffness matrix in order to control the memory and CPU requirements for simulating large systems with irregular meshes. Both requirements scales as at least $O\left(N^{2}\right)$ if the full stiffness matrix is retained, and this 
scaling can be reduced to $O(N)$ if matrix elements are discarded if $K_{i j}<\epsilon K_{i i}$ for some small number $\epsilon$. This approach allows the simulation of billion atom systems (greatly coarse-grained) on desktop workstations without approximation beyond those presented here.

For $T \neq 0$ the finite temperature dynamical matrix should be used for $D_{\mu \nu}$. This quasi-harmonic approximation ensures a consistent thermodynamics, and it effectively sums the two-legged diagrams of the finitetemperature anharmonic perturbation theory (i.e., those terms second order in the CG displacement). For example, in ergodic systems the time average of the kinetic energy term in the CG energy (29) is related to the temperature through a equipartition expression. In general, the dynamical matrix may depend on other macroscopic parameters, as well, such as slowly varying external magnetic and electric fields. $D_{\mu \nu}$ should be evaluated under these conditions. Also note that while the harmonic approximation may be good in peripheral regions, it may not be appropriate for the important regions. We have shown that the CGMD and MD equations of motion agree in regions where the mesh coincides with the atomic sites. In these regions, the full MD potential may, and should, be employed, so that effects such as diffusion and dislocation dynamics are allowed.

\section{A. Normal Modes and the Inverse of $D_{\mu \nu}$}

In order to simulate large CG regions, it is necessary to take some measures to increase computational efficiency. One such trick is to make use of the long-range order in a single crystal to facilitate the computation of the stiffness matrix. The eigenstates of the dynamical matrix $D_{\mu \nu}$ are plane waves. For monatomic lattices they correspond to the normal modes of the system, the longitudinal and transverse phonons in the acoustic and optical branches that are familiar from lattice dynamics $\stackrel{50,57}{5}$ In reciprocal space, where the basis elements are exactly these plane waves, the dynamical matrix is diagonal. The inverse of the dynamical matrix is then trivial to compute, and the subtlety of inverting a singular matrix is eliminated because reciprocal space naturally factorizes into a direct product of the three zero modes with $\mathbf{k}=0$ and all of the other modes with non-zero eigenvalues.

To be specific, the equation for the stiffness matrix (37) becomes

$K_{i j}^{a b}=\left(N N^{T}\right)_{i m}\left[N_{m}(-\mathbf{k}) D_{a b}^{-1}(\mathbf{k}) N_{n}(\mathbf{k})\right]^{-1}\left(N N^{T}(8,9)\right.$

where $\mathbf{k}=0$ is explicitly omitted from the sum. Now the inner matrix inverse is the inverse of a $3 \times 3$ matrix.

\section{B. Shape Functions}

This section discusses particular choices for interpolation functions. Compatible combinations of these are also allowed, as in FEM with wedge and brick elements, for example. We emphasize how different choices for interpolation functions meet the requirement of meshing the crystal lattice at the $\mathrm{MD} / \mathrm{CG}$ interface. The usual linear interpolation functions for tetrahedral elements 58 are the simplest functions meeting the three criteria (ii) - (iii) in Section III They are defined such that $N_{j}(\mathbf{x})$ is 1 at node $\mathbf{x}_{j}$, it goes linearly to zero at the nearestneighbor nodes, and it vanishes outside of the nearest cells. Suppose $\mathbf{x}$ is in the $k$ th element with nodes $\mathbf{x}_{k_{j}}$ where $j=1, \ldots, 4$. Then the interpolation functions are given by the volumnal or natural tetrahedral coordinates:

$$
\begin{aligned}
N_{k_{j}}(\mathbf{x}) & =\mathbf{x} \cdot \partial_{k_{j}} \log V_{k}\left(\mathbf{x}_{k_{1}}, \ldots, \mathbf{x}_{k_{4}}\right) \\
V_{k} & =\frac{1}{6}\left|\begin{array}{llll}
1 & x_{k_{1}} & y_{k_{1}} & z_{k_{1}} \\
1 & x_{k_{2}} & y_{k_{2}} & z_{k_{2}} \\
1 & x_{k_{3}} & y_{k_{3}} & z_{k_{3}} \\
1 & x_{k_{4}} & y_{k_{4}} & z_{k_{4}}
\end{array}\right|
\end{aligned}
$$

where $\mathbf{x}_{k}=\left(x_{k}, y_{k}, z_{k}\right)$ and we have written the tetrahedral volume as a determinant. The interpolation function for node $k_{j}$ is simply the volume of the tetrahedron formed by $\mathbf{x}$ and the other three nodes divided by the volume of the entire tetrahedral cell. These functions are clearly $\mathrm{C}^{0}$ continuous and independent. It is easily checked that they are linear and form a partition of unity. They also have the desirable properties of locality and ease of use. The locality property is particularly important for our applications, since the domains requiring an atomistic treatment are localized to small regions of the system.

Another basis we have found useful is the set of the longest wavelength normal modes. These functions satisfy the less stringent basis properties: $\left(\mathrm{i}^{\prime}\right)$ linear independence, $\operatorname{det} N_{j}\left(\mathbf{x}_{k}\right) \neq 0$, and ( $\left.\mathrm{ii}^{\prime}\right)$ representation of unity, $1=\sum_{j=1}^{N_{\text {node }}} c_{j} N_{j}(\mathbf{x})$ for some constants $c_{j}$. This basis provides a check of the CG Hamiltonian (3), since these functions are the optimal basis for a regular CG meshthe phonon spectrum comes out exactly correct, apart from the missing short-wavelength modes. The disadvantage of this basis for irregular meshes is that it is nonlocal and the short wavelength modes that should be supported on the finer parts of the mesh are absent. In particular, the stiffness matrix elements decrease as

$$
K_{i j} \sim \frac{1}{\Delta x_{i j}},
$$

instead of decreasing exponentially with distance in the local basis case. The short wavelength modes could be restored locally through the use of a wavelet basis, in principle, but we have not implemented a wavelet-based version of CGMD.

In many cases, higher order polynomial interpolating functions are the basis of choice. Generalizations of the 8 -node brick used for hexahedral lattices 58 are particularly easy to implement. For example, a generalized 8node brick is the element we used to calculate the CGMD spectra for solid argon and tantalum presented in Section 
VIIA below. First, consider a simple cubic lattice. The basic 8-node brick involves interpolations functions of the form 58

$$
N_{1}^{\text {cubic }}\left(\xi_{a}\right)=\frac{1}{8}\left(1+\xi_{1}\right)\left(1+\xi_{2}\right)\left(1+\xi_{3}\right)
$$

where throughout this section we use the scaled coordinates $\xi_{a} \in[-1,1) . N_{1}$ is associated with the corner node at $\vec{\xi}=(1,1,1)$. The other 7 interpolation functions are generated by the action of the point group $O_{h}$ on $N_{1}$.

The cubic elements may be applied to a variety of hexahedral elements by mapping the real space coordinates onto the $[-1,1)^{3}$ cube in the coordinate space using the standard multi-linear coordinate transformation often called "natural coordinates" for the hexahedron in the finite element literature 58 For our purposes, some especially important cases are the monatomic Bravais lattices, such as face-centered cubic (fcc) and body-centered cubic (bcc) lattices. Suppose $\mathbf{a}_{a}$ are the basis vectors in real space, and $\mathbf{b}_{a}$ are the reciprocal basis vectors such that $\mathbf{a}_{a} \cdot \mathbf{b}_{b}=\delta_{a b}$. For example, in the fcc lattice the basis vectors could be chosen to be $\mathbf{a}_{1}=\left(0, \frac{1}{2}, \frac{1}{2}\right) a, \mathbf{a}_{2}=$ $\left(\frac{1}{2}, 0, \frac{1}{2}\right) a$ and $\mathbf{a}_{3}=\left(\frac{1}{2}, \frac{1}{2}, 0\right) a$, and $\mathbf{b}_{1}=(-1,1,1) / a$, $\mathbf{b}_{2}=(1,-1,1) / a$ and $\mathbf{b}_{3}=(1,1,-1) / a$, where $a$ is the lattice constant. Then interpolation functions on the Bravais lattice are given by

$$
N_{j}^{\text {Bravais }}(\mathbf{x})=N_{j}^{\text {cubic }}\left(\xi_{a}=2 \mathbf{b}_{a} \cdot \mathbf{x}-1\right)
$$

using the shape functions defined in Eq. (93). Shape functions for the bcc lattice can be constructed in the same way, with the basis vectors be chosen to be $\mathbf{a}_{1}=$ $\left(\frac{1}{2}, \frac{1}{2},-\frac{1}{2}\right) a, \mathbf{a}_{2}=\left(\frac{1}{2},-\frac{1}{2}, \frac{1}{2}\right) a$ and $\mathbf{a}_{3}=\left(-\frac{1}{2}, \frac{1}{2}, \frac{1}{2}\right) a$, and $\mathbf{b}_{1}=(1,1,0) / a, \mathbf{b}_{2}=(1,0,1) / a$ and $\mathbf{b}_{3}=(0,1,1) / a$, where $a$ is the lattice constant.

One drawback of the fcc and bcc shape functions (94) is that they break the point group symmetry of the lattice. Acting on the mesh with an element of the point group returns a new mesh with the same nodes but often a different set of cell boundaries. One example is $C_{4}^{z}$, the $90^{\circ}$ rotation about $z$. It changes the cell boundaries, as can be seen by its action on $\frac{1}{2}\left(\mathbf{a}_{1}+\mathbf{a}_{2}\right)$ [i.e., $\vec{\xi}=\left(\frac{1}{2}, \frac{1}{2}, 0\right)$ ]. $C_{4}^{z}$ maps this face point to $\vec{\xi}^{\prime}=\left(1,0,-\frac{1}{2}\right)$, a point on an edge of the original mesh. Another way to understand this symmetry breaking is that we made a choice when we selected the basis $\mathbf{a}_{1}=\left(0, \frac{1}{2}, \frac{1}{2}\right) a, \mathbf{a}_{2}=\left(\frac{1}{2}, 0, \frac{1}{2}\right) a$ and $\mathbf{a}_{3}=\left(\frac{1}{2}, \frac{1}{2}, 0\right) a$. Had we selected another basis, say $\mathbf{a}_{1}^{\prime}=\left(-\frac{1}{2}, 0, \frac{1}{2}\right) a, \mathbf{a}_{2}^{\prime}=\left(0, \frac{1}{2}, \frac{1}{2}\right) a$ and $\mathbf{a}_{3}^{\prime}=\left(-\frac{1}{2}, \frac{1}{2}, 0\right) a$, then the mesh would have been different. It would have different cell edges and faces, even though the cell nodes would be the same. More importantly for our purposes, a displacement field interpolated using one set of shape functions cannot, apart from a few special cases, be represented exactly with the rotated shape functions. The results of CGMD modeling then depend to some extent on the choice of basis and associated shape functions.

The symmetry breaking is small and of no consequence in most applications; however, we are interested in us- ing wave spectra as a test of CGMD, plotting the spectra along high symmetry directions in the Brillouin zone. The symmetry breaking is somewhat troublesome in this case because high symmetry directions no longer possess the high symmetry and directions that are supposed to be equivalent by symmetry are not. We have developed a symmetrization procedure to eliminate the effects completely. Its use is limited to applications where high symmetry is important, such as wave spectra, so we present it below where the spectra are calculated.

Another approach to this problem is to introduce polynomial bases that respect the point group symmetry. The well-known serendipity functions ${ }^{21.59}$ are an example of a minimal polynomial basis that respects cubic symmetry. The serendipity functions may be generalized in a way that makes them suitable for a cubic fcc or bcc cell such that there is a node for each atom in the cubic unit cell, so that the MD degrees of freedom are recovered in the atomic limit. These are different than the usual serendipity functions and, indeed, are not as well suited for conventional FEM applications because of the location of the nodes (e.g. the bcc element has an internal node) ${ }^{21}$ They do, however, meet our need to match the atomic lattice and preserve symmetries in the atomic limit. For example, the face-centered cubic (fcc) serendipity functions have nodes at the corners and the at the middle of the faces of a cube. To the best of our knowledge, this kind of FEM interpolation function has not been used previously. They are well-suited to Bravais lattices, because of their simple action under the point group symmetry. Consider a cubic unit cell of an fcc lattice with local coordinates $\xi_{a}$, where $-1 \leq \xi_{a} \leq 1$ for $a=1,2,3$. Define the function

$$
\begin{aligned}
& N_{1}\left(\xi_{a}\right)= \frac{1}{8}\left(1+\xi_{1}\right)\left(1+\xi_{2}\right)\left(1+\xi_{3}\right) \times \\
& {\left[2\left(\xi_{1}+\xi_{2}+\xi_{3}-1\right)-\left(\xi_{1} \xi_{2}+\xi_{2} \xi_{3}+\xi_{3} \xi_{1}\right)\right] }
\end{aligned}
$$

associated with the corner node at $\vec{\xi}=(1,1,1)$ and the function

$$
N_{9}\left(\xi_{a}\right)=\frac{1}{2}\left(1+\xi_{1}\right)\left(1-\xi_{2}^{2}\right)\left(1-\xi_{3}^{2}\right)
$$

associated with the face node at $\vec{\xi}=(1,0,0)$. Basis functions, $N_{j}\left(\xi_{a}\right)$ associated with the other nodes are generated from (95) and (96) by the action of the point group. These functions satisfy the strong requirements for an interpolation basis. Each function vanishes outside of the cells containing the corresponding node, and it goes to zero at the opposite faces of those cells: it is local and continuous. Also, taken together they form a partition of unity, as is easily checked. These 14 functions comprise only part of the set of 26 polynomials of order at most $(2,2,1)$; i.e. the set of polynomials with terms no higher than $x^{2} y^{2} z$ (or $x^{2} y z^{2}$, etc.). But they are specified uniquely by the three basis requirements and the fact that they respect the point group; i.e. a point group operation which leaves a particular node invariant also leaves the corresponding function invariant. These fcc shape 
functions are most useful for testing purposes such as the computation of phonon spectra and scattering properties where it is desirable to maintain as many symmetries as possible.

Other (novel) fcc symmetric bases are possible if one abandons the notion of self-contained elements. Typically, equations of motion in finite elements are assembled element by element. In CGMD, the interaction between two nodes decreases exponentially with their separation. Since the interactions are not contained within an element (in fact there is no absolute cutoff to their range in principle), the equations of motion are not constructed element by element, and the role of the elements is simply to guide in the construction of an interpolation basis. So we can consider an fcc lattice of nodes as four interlaced simple cubic lattices. The interpolation function for the corner node at $\vec{\xi}=(1,1,1)$ is given by

$$
N_{1}\left(\xi_{a}\right)=\frac{1}{16}\left(1+\xi_{1}\right)\left(1+\xi_{2}\right)\left(1+\xi_{3}\right)\left(\xi_{1}+\xi_{2}+\xi_{3}-1\right)
$$

and the functions for the other corners follow from symmetry. This completely determines a basis set which satisfies the criteria of locality and continuity. It does not satisfy the partition of unity requirement in the strictest sense, since the uniform displacement mode is over-represented: it is represented once for each sublattice. A constraint must be introduced that the uniform displacement on each sublattice is equal to the mean displacement:

$$
\overline{\mathbf{u}}=\sum_{j} \mathbf{u}_{j}^{(p)} / N_{\text {node }} \quad \text { for all sublattices } p .
$$

Note that all the nodes are equivalent. This is possible when the nodes are in a Bravais lattice such as fcc, but it is not true of the fcc basis in (95) and (96).

Interpolation functions for other crystal lattices are also available, either because they exist already in the finite element literature or because they are easily generated. We consider a few cases here.

Interpolation functions for the bcc lattice may be constructed in a similar fashion. They are of the order $(2,2,2)$. In particular, the shape function for the center node $(0,0,0)$ is

$$
N_{9}\left(\xi_{a}\right)=\left(1-\xi_{1}^{2}\right)\left(1-\xi_{2}^{2}\right)\left(1-\xi_{3}^{2}\right)
$$

which is unity at the associated node and vanishes on each face of the cell. Then the shape functions associated with the corners of the cell are of the form

$$
N_{1}\left(\xi_{a}\right)=\frac{1}{8}\left[\left(1+\xi_{1}\right)\left(1+\xi_{2}\right)\left(1+\xi_{3}\right)-N_{9}\left(\xi_{a}\right)\right]
$$

where this particular function is associated with the node at $(1,1,1)$. The shape functions associated with the other corners are generated by the appropriate rotations of this function. Again, these shape functions satisfy the criteria of locality, partition of unity and continuity.

Finally, we consider a two-dimensional case that is relevant for many of the crystal lattices: the square lattice.
In particular, suppose that the $\mathrm{CG}$ region will be treated as a two-dimensional projection of the 3D lattice along the [001] direction. The square lattice has been used extensively in the literature and we include the minimal interpolation functions here for reference:

$$
N_{1}\left(\xi_{a}\right)=\frac{1}{4}\left(1+\xi_{1}\right)\left(1+\xi_{2}\right)
$$

These interpolation functions are not only useful for two-dimensional projections of the lattices treated above (simple cubic, fcc and bcc with [001] projection), but also other more complicated lattices such as the diamond cubic lattice with [001] projection.

\section{Shape Functions in Reciprocal Space}

In order to make use of the reciprocal space representation of the dynamical matrix, it is necessary to have the Fourier transform of the shape functions. The Fourier transform can be computed numerically, of course, using fast Fourier transform (FFT) techniques. In some cases it is also possible to compute the Fourier transform analytically. In this section, we derive the atomic-index Fourier transform of the linear interpolation function in one dimension. The result is immediately applicable to the 4-node square and the 8-node brick in two and three dimensions, respectively. The result is given in Eq. (110) below, and readers who are not interested in the derivation are free to skip to Section VID

Consider the symmetric linear interpolation function on a regular mesh in one dimension:

$$
N_{j}(x)= \begin{cases}1-\left|\frac{x-x_{j}}{x_{j+1}-x_{j}}\right| & \text { for }\left|x-x_{j}\right|<x_{j+1}-x_{j} \\ 0 & \text { otherwise. }\end{cases}
$$

Let $a$ be the lattice constant, and $N_{p e r}=\left(x_{j+1}-x_{j}\right) / a$. We first note the useful identities:

$$
\begin{aligned}
\sum_{\mu=0}^{N_{\text {per }}} e^{i k a \mu} & =e^{i k a N_{\text {per }} / 2} \frac{\sin \left[k a\left(N_{\text {per }}+1\right) / 2\right.}{\sin (k a / 2)}(103) \\
\sum_{\mu=-N_{\text {per }}}^{N_{\text {per }}} e^{i k a \mu} & =\frac{\sin \left[k a\left(2 N_{\text {per }}+1\right) / 2\right]}{\sin (k a / 2)}
\end{aligned}
$$

that follow from the well known formula to sum geometric series $\left[1+z+z^{2}+\ldots+z^{N}=\left(1-z^{N+1}\right) /(1-z)\right]$ together with de Moivre's formula.

We first transform the atomic index $\mu$ of the shape function to the Fourier conjugate variable $k$ :

$$
N_{j}(k)=\sum_{\mu} N_{j \mu} e^{i k a \mu} .
$$

The shape function $N_{j \mu}$ is expressed as the sum of two terms in Eq. (102): the transformation of the first term, just equal to unity, is given by Eq. (104), but the transformation of the second is more involved. It is calculated 
as follows:

$$
\begin{aligned}
& \sum_{\mu=-N_{p e r}}^{N_{p e r}}|\mu| e^{i k a \mu}=(2 / a) \partial_{k} \operatorname{Im}\left\{\sum_{\mu=0}^{N_{p e r}} e^{i k a \mu}\right\} \\
& =(2 / a) \partial_{k} \operatorname{Im}\left\{e^{i k a N_{p e r} / 2} \frac{\sin \left[k a\left(N_{\text {per }}+1\right) \text { ket } p\right.}{\sin (k a / 2)}\right\}^{\text {mode mate }}(107) \\
& =(2 / a) \partial_{k}\left[\sin ^{2}\left(k a N_{p e r} / 2\right) \cot (k a / 2)+\frac{1}{2} \sin \left(k a N_{k(1,0)}\right)\right]^{\epsilon_{\mu \nu}}=\sum_{a}\left(v_{a}\right)_{\mu}\left(v_{a}\right)_{\nu} \\
& =N_{p e r} \frac{\sin \left[k a\left(2 N_{p e r}+1\right) / 2\right]}{\sin (k a / 2)}-\frac{\sin ^{2}\left(k a N_{p e r} / 2\right)}{\sin ^{2}(k a / 2)} .
\end{aligned}
$$

We have developed an alternative resolution of the zero $\left(v_{a}\right)_{\mu}$ be(1496) $\mu$ th component of the ath zero mode of $D_{\mu \nu}$; i.e. $\sum_{\nu} D_{\mu \nu}\left(v_{a}\right)_{\nu}=0$ for $a=1,2,3$. Define the

Combining the two contributions we find

$$
N_{j}(k)=\frac{1}{N_{p e r}} \frac{\sin ^{2}\left(k a N_{p e r} / 2\right)}{\sin ^{2}(k a / 2)} e^{i k x_{j}}
$$

where $N_{p e r}$ is the number of lattice sites per CG cell. In higher dimensions, $N_{p e r}$ would be replaced by $N_{p e r}^{x}, N_{p e r}^{y}$ and $N_{p e r}^{z}$. This result applies to the regular CG lattice. The corresponding formula for a general one-dimensional CG lattice is much more complicated, and using a numerical FFT to calculate it is generally recommended.

\section{The Center-of-Mass Mode}

The stiffness matrix definition involves two matrix inverses. This is somewhat ill-defined because $D_{\mu \nu}$ is singular, due to the zero modes. The zero modes are the zero energy phonons at the $\Gamma$ point in reciprocal space that are associated with translation invariance of the center of mass. There are $d$ zero energy phonons in any $d$ dimensional system corresponding to uniform translation in each of the $d$ directions. These zero modes make the matrix singular. Since we have imposed the criterion that the center-of-mass mode should be represented within the set of interpolation functions, the singularity is superficial. There are two inverses in Eq. (36), the matrix $K_{i j}$ is finite after a suitable regularization. Indeed, the alternate derivation of the stiffness matrix given in Appendix A is free from any zero mode problems, so it must be possible to devise a suitable regularization scheme. An obvious example is

$$
K_{j k}=\lim _{\epsilon \rightarrow 0}\left(\sum_{\mu \nu} f_{j \mu}\left(D_{\mu \nu}+\epsilon I_{\mu \nu}\right)^{-1} f_{k \nu}\right)^{-1}
$$

where $I_{\mu \nu}$ is the identity matrix.

The regularization (111) is conceptually simple, but in practice a small but finite $\epsilon$ must be used, and error is introduced into the contribution of the long wavelength modes. The error can be controlled through the choice of an $\epsilon$ which is small enough that frequencies of interest are not affected appreciably, but large enough that the matrix is numerically well conditioned. This regularization is cheap and adequate for many purposes.
Using these matrices we construct the projected shape matrix

$$
\begin{aligned}
& \tilde{N}_{j \mu}=\sum_{k} \mathcal{P}_{j k} N_{k \mu} \\
& \mathcal{P}_{j k}=\delta_{j k}-\epsilon_{j k}^{\prime}
\end{aligned}
$$

Then the stiffness matrix (36) is given by the matrix equation

$$
K=\left(\tilde{N} \tilde{N}^{T}\right)\left[\tilde{N}(D+c \epsilon)^{-1} \tilde{N}^{T}+c^{\prime} \epsilon^{\prime}\right]^{-1}\left(\tilde{N} \tilde{N}^{T}\right)
$$

The nonzero numbers $c$ and $c^{\prime}$ are arbitrary, but should be comparable to the eigenvalues of $D_{\mu \nu}$ to make the matrices well-conditioned. This formula works by shifting the zero eigenvalues in the atomic space and those in the nodal space by $c$ and $c^{\prime}$, respectively, without affecting the other eigenvalues. The projection matrices undo this shift. They are needed within the brackets to stifle the crossterms between the zero modes and the nonzero modes for incommensurate meshes. For commensurate meshes, this formula simplifies to

$$
\begin{aligned}
K_{\text {commensurate }} & =\left(N N^{T}\right)\left[X-X \cdot \epsilon^{\prime}\right]\left(N N^{T}\right) \\
X & =\left[N(D+c \epsilon)^{-1} N^{T}\right]^{-1}
\end{aligned}
$$

where $X \cdot \epsilon^{\prime}$ is a symmetric matrix since $\sum_{\mu} N_{i \mu}\left(v_{a}\right)_{\mu}$ are eigenvectors of $X_{i j}$.

The zero modes are not integrated out, so a short ranged $D_{\mu \nu}$ results in a short ranged $K_{i j}$. On the other hand, a nearest neighbor $D_{\mu \nu}$ does not generally produce a nearest neighbor $K_{i j}$, except where the mesh is atomic sized. The stiffness matrix elements typically decrease exponentially with separation, so the effective interaction is short ranged but not nearest neighbor. This is an important point, since it is this quality that improves the CGMD phonon spectrum.

\section{VII. (QUASI-)HARMONIC CRYSTALS}

Various properties of harmonic crystals have been computed within CGMD as a validation of the methodology. 


\section{A. Phonon Spectra}

The CGMD phonon spectrum offers a good first test of the model. Consider a regular, but not necessarily commensurate CG mesh. The equations of motion for the Hamiltonian (29) are

$$
M_{i j} \ddot{u}_{j}^{a}=-K_{i l}^{a b} u_{l}^{b},
$$

where $\ddot{u}_{j}^{a}$ is the nodal acceleration of the $j^{t h}$ node in the $a^{\text {th }}$ direction. Substitution of a plane-wave normal mode $u_{j}^{a}(t)=u_{0}^{a} e^{i \mathbf{k} \cdot \mathbf{x}_{j}-i \omega t}$ produces the secular equation

$$
M(\mathbf{k}) \omega^{2} \delta_{a b}=K^{a b}(\mathbf{k})
$$

where $M(\mathbf{k})$ and $K^{a b}(\mathbf{k})$ are the Fourier transform of the mass and stiffness matrices, respectively. The form of the mass matrix for a monatomic lattice allows further simplification:

$$
m^{2} \omega^{2} \delta_{a b}=\left[M\left(N D^{-1} N^{T}\right)_{a b}^{-1}\right](\mathbf{k})
$$

where we have used Eqs. (32) and (37). For incommensurate meshes, we have calculated the right-hand side of Eq. (121) in real space, then found its Fourier transform and solved the secular equation for the phonon frequencies. One such spectrum is plotted in Fig. 1 of Ref. 17. In principle this could be done in any case, but the computational cost limits the size of systems that can be treated in this manner. We have done calculations with billions of atoms per CG cell, but in order to do this it is necessary to eliminate the real-space representation of the dynamical matrix.

For commensurate meshes with uniform mesh size, we may go to reciprocal space and the formulas simplify considerably. It is even possible to derive analytic formulas for the spectra in some cases. Suppose the CG mesh contains $N_{\text {node }}^{a}$ nodes in the $a^{t h}$ dimension for a total length of $L_{a}$. The CG shape functions in reciprocal space may be expressed in terms of a Bravais lattice character $\chi$ for the CG mesh and a CG element structure factor $S$ :

$$
\begin{aligned}
N\left(\mathbf{k}, \mathbf{k}^{\prime}\right) & =\sum_{j, \mu} e^{i \mathbf{k} \cdot \mathbf{x}_{j}-i \mathbf{k}^{\prime} \cdot \mathbf{x}_{\mu}} N_{j \mu} \\
& =\chi\left(\mathbf{k}-\mathbf{k}^{\prime}\right) S\left(\mathbf{k}^{\prime}\right) \\
|\chi(\delta \mathbf{k})| & =\prod_{a=1}^{3}\left(\frac{\sin \left(\delta k_{a} L_{a} / 2\right)}{\sin \left(\delta k_{a} L_{a} /\left(2 N_{\text {node }}^{a}\right)\right)}\right) \\
S\left(\mathbf{k}^{\prime}\right) & =\sum_{\mu \in \Omega_{j}} e^{-i \mathbf{k}^{\prime} \cdot\left(\mathbf{x}_{\mu}-\mathbf{x}_{j}\right)} N_{j \mu}
\end{aligned}
$$

where $\Omega_{j}$ is any one element from the CG mesh. Note that in the atomic limit $S$ is just a delta function in the first Brillouin zone. The CG phonon spectrum for a monatomic lattice is given by

$$
\begin{aligned}
\omega^{2}(\mathbf{k})= & {\left[\frac{1}{m} \sum_{k^{\prime}}\left|\chi\left(\mathbf{k}-\mathbf{k}^{\prime}\right)\right|^{2}\left|S\left(\mathbf{k}^{\prime}\right)\right|^{2}\right] \times } \\
& {\left[\sum_{k^{\prime}}^{\prime}\left|\chi\left(\mathbf{k}-\mathbf{k}^{\prime}\right)\right|^{2}\left|S\left(\mathbf{k}^{\prime}\right)\right|^{2}\left[D\left(\mathbf{k}^{\prime}\right)\right]^{-1}\right]^{-1} }
\end{aligned}
$$

The coarse-grained mass and stiffness matrices conspire to produce a Padé approximant of the true spectrum, and thereby achieve the $\mathcal{O}\left(k^{4}\right)$ improved relative error, compared to the $\mathcal{O}\left(k^{2}\right)$ relative error of the two FEM spectra. where $D\left(\mathbf{k}^{\prime}\right)$ is a $3 n \times 3 n$ matrix where $n$ is the number of atoms in the unit cell, and the two inverses are matrix inverses. The frequencies are the eigenvalues of the resulting matrix. As in Eq. (121), the first term represents the mass matrix in reciprocal space divided by $\mathrm{m}^{2}$; the second term is the middle factor of the stiffness matrix. In the atomic limit, the formula reduces to the usual expression, $D(k) / m$.

\section{B. Analytic Formula for CG Spectrum}

The CGMD spectrum (126) may be computed in closed form for a monatomic solid with a commensurate CG mesh in one dimension. We presented the analytic expression for the spectrum with nearest neighbor interactions and linear interpolation in Ref. 17, Eq. (12):

$$
\omega(k)=2 \sqrt{\frac{K}{m}}\left(\frac{\sum_{p} \sin ^{-4}\left(\frac{1}{2} k a+\frac{\pi p}{N_{p e r}}\right)}{\sum_{p} \sin ^{-6}\left(\frac{1}{2} k a+\frac{\pi p}{N_{p e r}}\right)}\right)^{1 / 2}
$$

where the sums over $p$ run from 0 to $N_{\text {per }}-1, N_{\text {per }}=$ $N_{\text {atom }} / N_{\text {node }}$ is the number of atoms per cell and $K$ is the nearest-neighbor spring constant. This formula shows the contribution of many modes of the underlying crystal to each CGMD mode, resulting from the choice of interpolation functions which have many normal mode components. Near the center of the CG Brillouin zone, a single mode $(p=0)$ dominates the sums (127). This dominance reflects the fact that long wavelength modes are well represented on the CG mesh. Near the boundary of the $\mathrm{CG}$ zone $\left[k \approx N_{\text {node }} \pi /(N a)\right]$, many modes contribute. The many modes are needed because periodicity forces the slope of the spectrum to zero, and the modes act in concert to keep the CGMD spectrum close to the true spectrum which is not smooth at the boundary. For comparison, the formula for the lumped mass FEM spectrum is

$$
\omega^{\operatorname{lump}}(k)=2 \sqrt{\frac{K}{m}} \frac{1}{N_{p e r}} \sin \left|\frac{1}{2} k N_{p e r} a\right|,
$$

the formula for the FEM spectrum with the distributed (consistent) mass matrix is

$$
\omega^{\mathrm{dist}}(k)=2 \sqrt{\frac{K}{m}} \frac{1}{N_{p e r}} \frac{\sin \left|\frac{1}{2} k N_{p e r} a\right|}{\sqrt{1-\frac{2}{3} \sin ^{2}\left(\frac{1}{2} k N_{p e r} a\right)}}
$$

and the formula for the exact MD spectrum is

$$
\omega^{\mathrm{MD}}(k)=2 \sqrt{\frac{K}{m}} \sin \left|\frac{1}{2} k a\right| .
$$


The remainder of this subsection is devoted to the derivation of the analytic formula for the CGMD spectrum (127). We make use of the formula for the Fourier transform of the symmetric linear interpolation function on a regular mesh in one dimension, given above in Eq. (110):

$$
N_{j}\left(k^{\prime}\right)=\frac{1}{N_{p e r}} \frac{\sin ^{2}\left(k^{\prime} a N_{p e r} / 2\right)}{\sin ^{2}\left(k^{\prime} a / 2\right)} e^{i k^{\prime} x_{j}}
$$

where in this formula only the atomic index has been transformed. The Fourier transform of the index $j$ is straightforward, and the spectrum could then be derived using Eq. (126). We take a different approach. The spectrum is given by

$$
\begin{aligned}
\omega(k) & =\sqrt{\frac{K(k)}{M(k)}} \\
& =\sqrt{\frac{1}{m}} \sqrt{\frac{\left(N N^{T}\right)_{k}}{\left(N D^{-1} N^{T}\right)_{k}}}
\end{aligned}
$$

where we have made use of the formulas for the stiffness and mass matrices, Eqs. (37) and (33), respectively. The subscript $k$ denotes the Fourier transform of wavenumber $k$, and we note that the Fourier transforms of both the nodal indices and the atomic indices take on values of the form $2 \pi n / L$, but for the atomic indices $-\frac{1}{2} L / a<n \leq \frac{1}{2} L / a$ whereas the Fourier transform of the nodal index lives in a reduced Brillouin zone, $-\frac{1}{2} N_{\text {node }}<n \leq \frac{1}{2} N_{\text {node }}$, where $N_{\text {node }}$ is the number of nodes (in one dimension).

Evidently, we need to calculate quantities of the form $\left(N X N^{T}\right)_{k}$, where the matrix $X$ is either the identity matrix or the inverse of the dynamical matrix. Such quantities are calculated in the following way:

$$
\begin{aligned}
\left(N X N^{T}\right)_{k}= & N_{\text {nodes }}^{-1} \sum_{i, j} e^{-i k\left(x_{i}-x_{j}\right)}\left(N X N^{T}\right)_{i j}(133) \\
= & N_{p e r}^{-1} \sum_{\Delta j=0}^{N_{\text {node }}-1} \sum_{k^{\prime}} e^{-i\left(k-k^{\prime}\right) \Delta j N_{p e r} a} \times \\
& X\left(k^{\prime}\right)\left[\frac{\sin ^{2}\left(k^{\prime} a N_{p e r} / 2\right)}{\sin ^{2}\left(k^{\prime} a / 2\right)}\right]^{2} \\
= & N_{p e r}^{-1} \sin ^{4}\left(\frac{1}{2} k^{\prime} a N_{p e r}\right) \times \\
& \sum_{p=0}^{N_{p e r}-1} \frac{X\left(k+\frac{2 \pi p}{N_{\text {per }} a}\right)}{\sin ^{4}\left(\frac{1}{2} k a+\frac{\pi p}{N_{p e r}}\right)}
\end{aligned}
$$

where Eq. (134) follows from Eq. (110), and we have used $x_{i}-x_{j}=(\Delta j) N_{\text {per }} a$ where $\Delta j=i-j$. To get from (134) to (135), we have used the fact that the sum over $\Delta j$ gives a delta function in the reduced Brillouin zone; i.e., a sum of delta functions periodically repeated through the full Brillouin zone.
The CGMD spectrum is then calculated using Eq. (132) together with Eq. (135) with $X$ equal to the identity in the numerator and $D^{-1}$ in the denominator. The result is

$\omega(k)=\sqrt{\frac{1}{m}}\left(\frac{\sum_{p} \sin ^{-4}\left(\frac{1}{2} k a+\frac{\pi p}{N_{p e r}}\right)}{\sum_{p} \sin ^{-4}\left(\frac{1}{2} k a+\frac{\pi p}{N_{p e r}}\right) D^{-1}\left(k+\frac{2 \pi p}{N_{p e r} a}\right)}\right)^{1 / 2}$

where $D(k)$ is the dynamical matrix in $k$-space and the sums over $p$ run from 0 to $N_{p e r}$. For a nearest-neighbor harmonic model $D(k)=4 K \sin ^{2}\left(\frac{1}{2} k a\right)$. Substitution of this into Eq. (136) results in the analytic formula for the CGMD spectrum that appears above (127). This calculation may be generalized to $3 \mathrm{D}$, where $D(k)$ is a $3 \times 3$ matrix and the shape functions are products of linear interpolations functions in each of the three dimensions.

The first test is the phonon spectrum for atoms with harmonic interactions coarse grained to a regular, but not necessarily commensurate mesh. The normal modes are plane waves both on the underlying ring of atoms and on the CG mesh. The wave vector $\mathbf{k}$ is a good quantum number for both. The nonzero terms of the dynamical matrix are of the form: $D_{\mu \mu}=2 K, D_{\mu, \mu \pm 1}=-K$. Figure 1 of Ref. 17 shows the resulting phonon spectra in four cases: exact, CGMD, distributed mass FEM and lumped mass FEM ${ }^{60}$ The latter two use the long wavelength elastic constants. The spectra are for a periodic chain of 1024 atoms with lattice constant $a$ coarse grained to 30 nodes.

Figure 1 of Ref. 17 shows that CGMD gives a better approximation to the true phonon spectrum than the two kinds of FEM do. All three do a good job at the longest wavelengths, as expected, but CGMD offers a higher order of accuracy. The relative error for CGMD is $\mathcal{O}\left(k^{4}\right)$ while that of the two versions of FEM is only $\mathcal{O}\left(k^{2}\right)$. At shorter wavelengths, there are significant deviations from the exact spectrum. The worst relative error of CGMD is about $6 \%$, better by more than a factor of three than that for FEM. This improvement is made possible by the longer-ranged interactions of CGMD as compared to FEM. The continuity condition satisfied by linear interpolation is enough to ensure that the hydrodynamic modes $(k \sim 0)$ are well modeled, but the lack of continuity of the derivatives shows up as error in the spectrum of the modes away from the zone center. This error vanishes for the smooth, nonlocal basis consisting of the longest wavelength normal modes. It turns out that the CGMD error at the CG zone boundary is relatively small (less than 1\%) for technical reasons. Also note that even though the number of atoms varies from cell to cell in the incommensurate mesh, the CGMD spectrum is free of anomalies. Other computations have shown that CGMD with linear interpolation is well behaved on irregular meshes, as well.

We are now in a position to investigate the effect of the CGMD relaxation terms eliminated in R-CGMD. They make the CGMD stiffness matrix non-local and therefore add to the cost of CGMD. What is the benefit of this additional computational complexity? Using the same pro- 


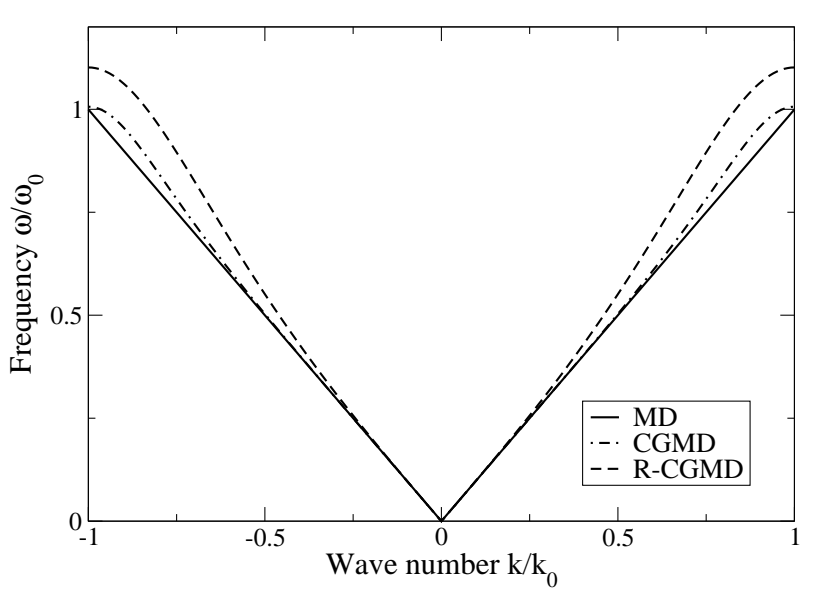

FIG. 5: A comparison of the acoustic wave spectra for CGMD, R-CGMD and MD with frequency vs. wavenumber plotted. The units are $k_{0}=\pi /\left(N_{p e r} a\right)$ and $\omega_{0}=k_{0} a \sqrt{K / m}$. The MD spectrum, corresponding to a simple 1D ball and spring model, is the ideal case. The CGMD and R-CGMD spectra are computed on a regular mesh with $N_{\text {per }}=32$ atoms per cell. Both full CGMD and its rigid approximation, RCGMD, are in good agreement with the MD spectrum for $k \sim 0$; i.e., at long wavelengths. At short wavelengths near the zone boundary, the CGMD spectrum is more accurate than the R-CGMD spectrum, a property that we attribute to the relaxation effects accounted for by CGMD but eliminated in the rigid approximation.

cedure outlined above, we have computed the R-CGMD spectrum:

$$
\begin{aligned}
\omega(k) & =\sqrt{\frac{1}{m}} \sqrt{\frac{\left(N D N^{T}\right)_{k}}{\left(N N^{T}\right)_{k}}} \\
& =\sqrt{\frac{1}{m}}\left(\frac{\sum_{p} \sin ^{-4}\left(\frac{1}{2} k a+\frac{\pi p}{N_{p e r}}\right) D\left(k+\frac{2 \pi p}{N_{p e r} a}\right)}{\sum_{p} \sin ^{-4}\left(\frac{1}{2} k a+\frac{\pi p}{N_{p e r}}\right)}\right)^{1 / 2}
\end{aligned}
$$

where the two lines are to be compared with the CGMD results (132) and (136), respectively. The CGMD and RCGMD spectra are plotted together with the MD spectrum in Fig. 5. It is clear that both approaches work well for long wavelengths $(k \sim 0)$. In fact, it is reasonable that the relaxation should be unimportant in this regime since the displacement field is varying slowly on the scale of the mesh, so the lowest energy configurations of the MD best fits to the interpolated displacement field should be close to having the atoms at their linearly interpolated positions. At short wavelengths (near the CG zone boundary), the story is different, however. The displacement field is varying at the scale of the mesh, and the atoms can reduce the energy through relaxation. This is evident in the improved value of the zone boundary frequency for CGMD ( $0.67 \%$ error) vs. R-CGMD (10.2\% error). As shown in Fig. 6] these values are typical, and close to the asymptotic value for coarse meshes. This difference in the performance of CGMD and R-CGMD is evident in many properties sensitive to the coarse-grained lattice

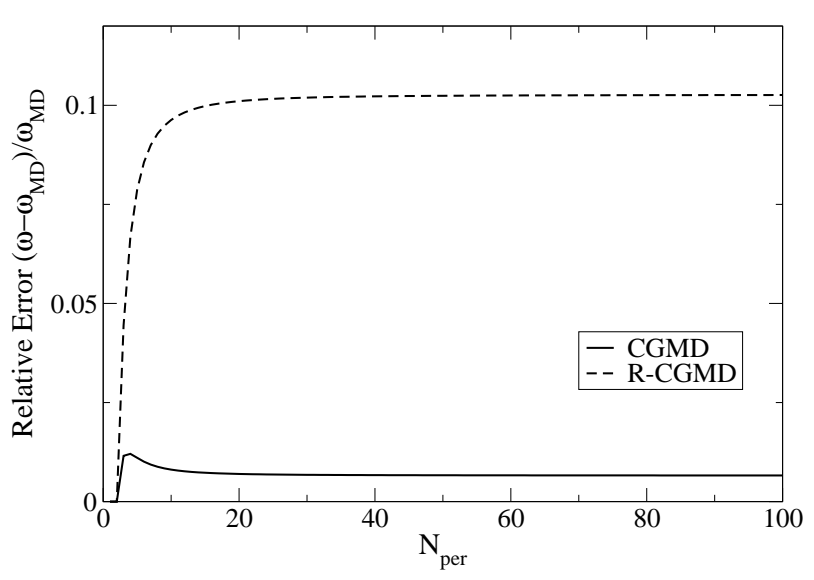

FIG. 6: A comparison of the error in the acoustic wave frequency at the zone boundary $\left[k=\pi /\left(N_{p e r} a\right)\right]$ for CGMD and R-CGMD as a function of the level of coarse graining, as expressed by $N_{p e r}$. At $N_{p e r}=1$, there is no error in either frequency. At all other values of $N_{p e r}$, the error in the CGMD frequency is less than that of R-CGMD, asymptotically going to $0.66 \%$ and $10.3 \%$, respectively.

dynamics at the zone boundary, such as the scattering properties we consider below. It is interesting to note that while the magnitude of the error is appreciably different, it is small in both cases. This suggests that there may be ways to formulate an approximation to CGMD that is intermediate between CGMD and R-CGMD, both in terms of the detail of the physics that is described and the computational cost. This is a topic we will address in the future.

\section{Numerical Calculation of Argon CG Spectrum}

It was shown in Ref. 17 that the CGMD phonon spectrum is closer to the true spectrum than that of FEM for a one dimensional chain of atoms with nearest-neighbor interactions. We now compare phonon spectra for a three dimensional real material: solid argon. Some of these results were reviewed briefly in Ref. 3. We also treat tantalum below. We find that CGMD again offers an improved spectrum.

Solid argon crystallizes in the fcc structure where it is well-described by the Lennard-Jones potential

$$
V=4 \epsilon\left(\frac{1}{(r / \sigma)^{12}}-\frac{1}{(r / \sigma)^{6}}\right)
$$

where $\epsilon=1.63 \times 10^{-21} \mathrm{~J}$ and $\sigma=3.44 \AA$.61 The elastic constants for this potential are given by $C_{11}=$ $105.3 \epsilon / \sigma^{3}=4.21 \mathrm{GPa}$ and $C_{12}=C_{44}=60.18 \epsilon / \sigma^{3}=$ $2.41 \mathrm{GPa}$.

We use the four-fold symmetrized 8-node brick interpolation functions defined on the fcc Bravais lattice for both CGMD and FEM. The fcc lattice is generated by the unit cell vectors $\mathbf{a}_{1}=\left(0, \frac{a}{2}, \frac{a}{2}\right), \mathbf{a}_{2}=\left(\frac{a}{2}, 0, \frac{a}{2}\right)$, and 
$\mathbf{a}_{3}=\left(\frac{a}{2}, \frac{a}{2}, 0\right)$. As discussed above, the conventional rhombohedral interpolation functions of the 8-node brick consist of products of one-dimensional linear interpolation functions:

$$
N_{j}(\mathbf{x})=\prod_{a=1}^{3} \tilde{N}\left[2 \mathbf{b}_{a} \cdot\left(\mathbf{x}-\mathbf{x}_{j}\right)-1\right]
$$

as in Eq. (94) These interpolation functions break the cubic symmetry. Even though the effect of the symmetry breaking is small, it complicates the analysis of the spectrum, causing deviations from the true spectrum that have nothing to do with the intrinsic accuracy of the methods.

In order to eliminate the small symmetry-breaking effects completely, we introduce a symmetrized version of the interpolation functions that is useful for spectrum calculations. We construct a multiplet of interpolated fields, where each component of the multiplet is an image of the mesh associated with Eq. (140) under the action of the point group. For the fcc lattice with rhombohedral cells, there are four inequivalent transformations of the mesh, so our field becomes a four component vector. The frequency values are then averaged over these four components. This is equivalent to the standard group theoretic operation of averaging over the orbit in order to restore symmetry. The four inequivalent group operations are the $C_{2}$ elements

$$
g=\left(\begin{array}{ccc} 
\pm 1 & 0 & 0 \\
0 & \pm 1 & 0 \\
0 & 0 & \pm 1
\end{array}\right)
$$

with -1 appearing an even number of times such that $g$ is a proper rotation with $\operatorname{det}(g)=1$. Another way to view this symmetrization procedure is that a symmetrized stiffness matrix is used

$$
K_{a b}^{s y m}(\mathbf{k})=\frac{1}{4} \sum_{g} g_{a a^{\prime}} K_{a^{\prime} b^{\prime}}(g \mathbf{k}) g_{b b^{\prime}}
$$

where the sum is over the four diagonal matrices $g$ (141). If the meshing did not break the point group symmetry, there would be no need for symmetrization and, indeed, the sum in Eq. (142) would reduce to a single term. The symmetrization procedure is simply a way to restore the symmetry broken by the mesh in order to facilitate analysis and comparison of the spectra.

We now undertake the actual calculation of the stiffness and mass matrices. The FEM matrices are computed as follows. The mass matrix takes one of two forms. The mass matrix computed strictly from the interpolation functions is known as the distributed or consistent mass matrix. It is given by

$$
\begin{aligned}
M_{i j}^{\text {dist }} & =\int d^{3} x \rho N_{i}(\mathbf{x}) N_{j}(\mathbf{x}) \\
& =\frac{1}{8} m N_{p e r}^{3} \int_{-1}^{1} d^{3} \xi N_{i}\left(\xi_{a}\right) N_{j}\left(\xi_{b}\right) \\
& =m N_{p e r}\left(\frac{2}{3}\right)^{3-l}\left(\frac{1}{6}\right)^{l}
\end{aligned}
$$

where $l(0 \leq l \leq 3)$ is the number of edges in the shortest path along a single hexahedral cell connecting nodes $i$ and $j$. The number $N_{p e r}$ is a generalization of the onedimensional case, where now it is the number of lattice sites along one dimension of the cell, and for cells with different dimensions in the three directions $N_{\text {per }}^{3}$ should be replaced by $N_{p e r}^{x} N_{p e r}^{y} N_{p e r}^{z}$. The Fourier transform of $M_{i j}^{\text {dist }}$ may then be calculated as a sum over the 27 neighboring nodes (indexed by $n_{1}, n_{2}, n_{3}$ running from -1 to 1$)$

$M^{\text {dist }}(\mathbf{k})=\frac{8 m N_{p e r}^{3}}{27} \sum_{n_{a}=-1}^{1}\left(\frac{1}{4}\right)^{\sum\left|n_{a}\right|} \prod_{a=1}^{3} \cos \left(N_{p e r} n_{a} \mathbf{a}_{a} \cdot \mathbf{k}\right)$

where $\mathbf{a}_{b}$ is the $b^{\text {th }}$ real space basis vector.

For many applications, it is sufficient (and in some cases even more accurate) to use a diagonal approximation to the mass matrix known as the lumped mass matrix. It is given by

$$
M_{i j}^{\text {lump }}=\delta_{i j} m N_{p e r}^{3}
$$

so each element of the diagonal is just equal to the mass contained in the Voronoi cell about the corresponding node. The Fourier transform of $M_{i j}^{\text {lump is }}$

$$
M^{\text {lump }}(\mathbf{k})=m N_{p e r}^{3}
$$

Note that $M^{\text {dist }}(\mathbf{k}=0)=M^{\text {lump }}(\mathbf{k}=0)$, and they are equal to the Voronoi mass as they should be.

The FEM stiffness matrix is given by

$$
\begin{aligned}
K_{i j ; b d}^{F E} & =C_{a b c d} b_{a a^{\prime}} b_{c c^{\prime}} \int d^{3} x \partial_{a^{\prime}} N_{i}(\mathbf{x}) \partial_{c^{\prime}} N_{j}(\mathbf{x}) \\
& =\frac{a}{8} N_{p e r}^{3} C_{a b c d} b_{a a^{\prime}} b_{c c^{\prime}} \int_{-1}^{1} d^{3} \xi \partial_{a^{\prime}} N_{i}(\xi) \partial_{c^{\prime}} N_{j}(\xi)
\end{aligned}
$$

where the prefactor $a$ is the lattice constant of the rhombohedral unit cell and the elastic tensor $C_{a b c d}$ has been contracted with the reciprocal space metric as appropriate for the non-orthogonal coordinates. This equation has too many components to present the complete expression here [cf. Ref. 58]. Nevertheless, the calculation is elementary algebra, and the results were used to calculate the Fourier transform. The result is a $3 \times 3$ matrix for each value of $k: K_{b d}^{F E M}(\mathbf{k})$. 
The formulas for the CGMD mass and stiffness matrices in real space for rhombohedral elements are computed similarly. The mass matrix is given by

$$
\begin{aligned}
M_{i j}^{\mathrm{CGMD}=} & m N_{p e r}^{3}\left(\frac{1-N_{p e r}^{-2}}{6}\right)^{l} \times \\
& \left(\frac{2+N_{p e r}^{-2}}{3}\right)^{3-l}
\end{aligned}
$$

where as in Eq. (145), $l(0 \leq l \leq 3)$ is the number of edges in the shortest path along a single rhombohedral cell connecting nodes $i$ and $j$. The correspondence of the leading terms to the terms in the FEM distributed mass matrix (145) is evident, so that CGMD reproduces the FEM distributed mass matrix in the large- $N_{\text {per }}$ limit. This expression assumes that the mesh consists of trigonal cells in which the linear dimensions are equal in all three dimensions, but it could be generalized immediately to unequal dimensions. The Fourier transform is given by

$$
\begin{aligned}
M^{\mathrm{CGMD}}(\mathbf{k})= & m N_{\text {per }}^{3} \sum_{n_{a}=-1}^{1}\left(\frac{1-N_{\text {per }}^{-2}}{6}\right)^{\sum\left|n_{a}\right|} \times \\
& \left(\frac{2+N_{\text {per }}^{-2}}{3}\right)^{3-\sum\left|n_{a}\right|} \prod_{a=1}^{3} \cos \left(N_{p e r} n_{a} \mathbf{a}_{a} \cdot \mathbf{k}\right)
\end{aligned}
$$

where as in Eq. (146), the 27 neighboring nodes are indexed by $n_{1}, n_{2}, n_{3}$ running from -1 to 1 . Note that the CGMD mass matrix also satisfies the mass sum rule: $M^{\mathrm{CGMD}}(\mathbf{k}=0)=m N_{\text {per }}^{3}$.

The CGMD stiffness matrix is calculated according to Eq. (37) using the reciprocal space representation of the dynamical matrix. In particular, we calculate the spectrum using Eq. (132), suitably generalized to a monatomic lattice in three dimensions:

$$
\omega_{a b}^{2}(\mathbf{k})=\frac{1}{m^{2}} M(\mathbf{k})\left(N D^{-1} N^{T}\right)^{-1}(\mathbf{k})
$$

where the actual frequency on each phonon branch is given by the square root of one of the three eigenvalues of the $3 \times 3$ matrix $\omega_{a b}^{2}(\mathbf{k})$. Here we have made use of the expression for the mass matrix of a monatomic lattice (32). The denominator is part of the stiffness matrix,

$$
\begin{aligned}
\tilde{K}_{a b} & =\left(N D_{a b}^{-1} N^{T}\right)^{-1}(\mathbf{k}) \\
& =\left(N\left(\mathbf{k}, \mathbf{k}^{\prime}\right) D_{a b}^{-1}\left(\mathbf{k}^{\prime}\right) N^{*}\left(\mathbf{k}, \mathbf{k}^{\prime}\right)\right)^{-1}
\end{aligned}
$$

where $D_{a b}^{-1}\left(\mathbf{k}^{\prime}\right)$ is the matrix inverse of the $3 \times 3$ matrix $D_{a b}\left(\mathbf{k}^{\prime}\right)$. The outer inverse is a $3 \times 3$ matrix inverse, as well. The Fourier transform of the shape functions is found using Eq. (110) to be

$$
\begin{aligned}
\left|N\left(\mathbf{k}, \mathbf{k}^{\prime}\right)\right|^{2}= & N_{\text {per }}^{3} \prod_{b=1}^{3} \sum_{p_{b}=1}^{N_{p e r}} \frac{\sin ^{4}\left(\mathbf{a}_{b} \cdot \mathbf{k} N_{\text {per }} / 2\right)}{N_{p e r}^{4} \sin ^{4}\left(\mathbf{a}_{b} \cdot \mathbf{k} / 2\right)} \times \\
\delta & \left(\mathbf{k}-\mathbf{k}^{\prime}+\frac{2 \pi p_{b}}{L_{b}}\right)
\end{aligned}
$$

where $L_{b}$ is the length of the CG cell in the $b^{\text {th }}$ direction. Upon substitution back into Eq. (153), we find that

$$
\begin{aligned}
\omega_{a b}^{2}(\mathbf{k})= & \left\{\sum_{p_{b}=1}^{N_{p e r}} \prod_{b=1}^{3}\left[\frac{\sin ^{4}\left(\mathbf{a}_{b} \cdot \mathbf{k} N_{p e r} / 2\right)}{N_{p e r}^{4} \sin ^{4}\left(\mathbf{a}_{b} \cdot \mathbf{k}_{p} / 2\right)}\right] D_{a b}^{-1}\left(\mathbf{k}_{p}\right)\right\}^{-1} \times \\
& M(\mathbf{k}) /\left(m^{2} N_{p e r}^{3}\right)
\end{aligned}
$$

where $\mathbf{k}_{p}=\mathbf{k}+2 \pi p_{b} / L_{b}$. The mass matrix $M(\mathbf{k})$ is given by Eq. (152). It is in the numerator, what might seem to be the wrong place, because of the form of the monatomic secular equation (121). It is clear from Eq. (157) that CGMD reproduces the MD spectrum in the long wavelength limit

$$
\omega_{a b}^{2}(\mathbf{k}) \sim D_{a b}(\mathbf{k}) / m \text { for } \mathbf{k} \sim 0
$$

which follows from expanding $\sin (x)=x+\ldots$ for small arguments. In the short wavelength limit the many terms in the sum over $p_{b}$ contribute to Eqs. (156) and (157), ensuring periodicity in the CG reciprocal space.

The spectrum is then computed in each case from the resulting secular equation at each value of $\mathbf{k}$. For the true spectrum, the secular equation is

$$
\operatorname{det}\left[\omega^{2}(\mathbf{k}) \delta_{a b}-D_{a b}(\mathbf{k}) / m\right]=0,
$$

for the CGMD spectrum the secular equation is

$$
\operatorname{det}\left[\omega^{2}(\mathbf{k}) \delta_{a b}-\omega_{a b}^{2}(\mathbf{k})\right]=0
$$

with $\omega_{a b}^{2}$ given by Eq. (157), and for the FEM spectra the secular equation is

$$
\operatorname{det}\left[\omega^{2}(\mathbf{k}) \delta_{a b}-K_{a b}(\mathbf{k}) / M(\mathbf{k})\right]=0
$$

with $M(\mathbf{k})$ given by Eqs. (146) and (148) for distributed and lumped mass, respectively. The determinant of the $3 \times 3$ matrix gives a cubic secular equation with three eigenvalues. The eigenvalues are real for all of the cases considered here. The Lennard-Jones potential for argon was cut off in real space after the twelfth nearest neighbor shell for both the MD and CGMD spectra. For many applications it would not be necessary to extend the range this far; however, in this case we wanted to test a potential extending well beyond nearest neighbors. The semi-analytic formulas that we have presented here, such as Eq. (157), have been used in order to calculate the spectra of extremely large systems with minimal computational expense, and all of the plots presented below were calculated using these equations. It should be 


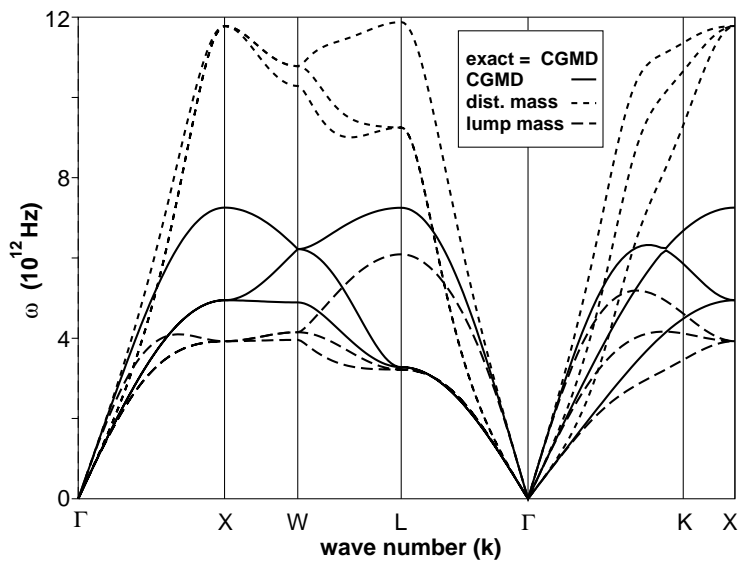

FIG. 7: The phonon spectra for solid argon in the atomic limit are shown as plots of wave frequencies vs. wave number along various high symmetry lines through the Brillouin zone ${ }^{3}$ The high symmetry points are labeled according to the standard convention for an fcc lattice ${ }^{62.63}$; for example, $\Gamma$ is at the center of the zone, $\mathbf{k}=0$ and $X=(1,0,0) \pi / a$ where $a$ is the fcc lattice constant. The 9 curves represent the 3 branches of the spectra for CGMD and two versions of FEM, one using the conventional distributed mass matrix and one using a diagonal lumped mass matrix. In the atomic limit CGMD reproduces the Lennard-Jones MD spectra exactly, whereas the FEM spectra show significant error, especially with the distributed mass matrix.

emphasized, however, that the spectra could have been calculated using the basic real-space matrix formulas or using numerical Fourier transforms. This has been done for the smaller systems as a validation.

The spectra have been computed for three levels of coarse-graining: the atomic limit $(1 \times 1 \times 1$ or no coarsegraining), a slight coarse-graining $(2 \times 2 \times 2)$ and a case approaching the continuum limit $(32 \times 32 \times 32)$. The numbers $n_{x} \times n_{y} \times n_{z}$ indicate the number of atoms within an unsymmetrized CG cell in each direction, i.e., $N_{\text {per }}=$ $1,2,32$, respectively. These values correspond to cells containing 1, 8 and 32678 atoms, respectively. The results are shown in Figs. 78 and 9

Elastic wave spectra are of interest because they provide a means of quantifying the small-amplitude dynamics of the system. They represent the energetics of every normal mode of vibration of a system of atoms. In coarsegrained dynamics, the wave spectra provide an excellent way to quantify the fidelity of the coarse-grained model. Since the normal modes of a crystal are plane waves, they are uniquely identified by a wave number $\mathbf{k}$ and a branch index, for example indicating a transverse optical mode or a longitudinal acoustic mode. The normal modes correspond to a lattice of points in reciprocal space (k-space) inside a bounded region known as the Brillouin zone. It is not possible to plot $\omega(\mathbf{k})$ for $\mathbf{k}$ throughout the three dimensional Brillouin zone, so typically $\omega(\mathbf{k})$ is plotted along lines, in particular high symmetry lines, through the $3 \mathrm{D}$ Brillouin zone ${ }^{62.63}$ This convention has been used

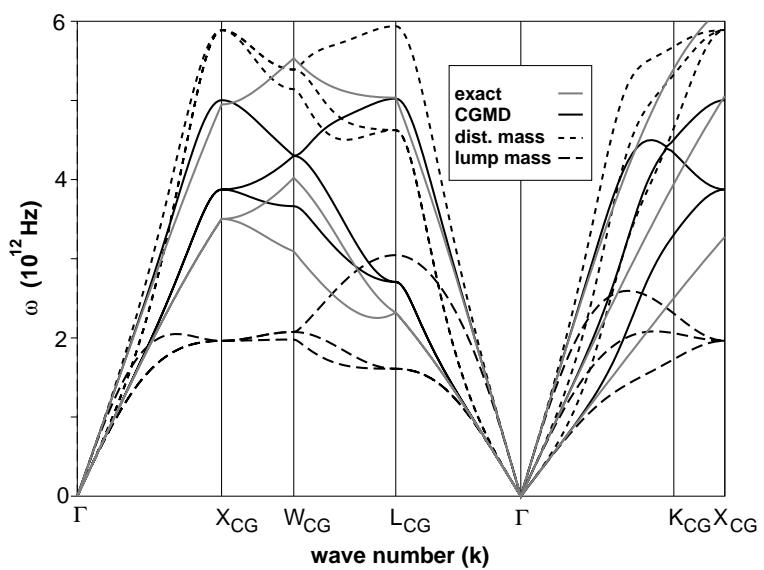

FIG. 8: The phonon spectra for solid argon on a mesh with slight coarse graining are shown as plots of wave frequencies vs. wave number along various high symmetry lines through the Brillouin zone (cf. the caption to Fig. 17). The 12 curves represent the 3 branches of the spectra for MD, CGMD and two versions of FEM, one using the conventional distributed mass matrix and one using a diagonal lumped mass matrix. Each cell of the CG mesh contains 8 atoms. For this first level of coarse graining the CGMD spectra is in better agreement with the MD spectra than either of the FEM spectra. Of the two FEM spectra, the lumped mass spectra is somewhat better.

\section{in Figs. 7 , 8 and 9}

Consider the spectra in the atomic limit shown in Fig. 7 The CGMD spectrum agrees precisely with the true spectrum. Of the two FEM spectra, the lumped mass spectrum is closer to the true spectrum. This is sensible because the mass is localized to the nodes in the atomic limit, since each node represents one atom. Overall, the lumped mass frequencies are lower than the true frequencies, whereas the distributed mass frequencies are higher. This ordering remains true regardless of the level of coarse-graining.

In the continuum limit shown in Fig. 9 the CGMD spectrum no longer agrees exactly with the true spectrum, but it is still a better approximation than either FEM spectrum. It is clear that in the continuum limit, the distributed mass produces the better spectrum of the two finite element cases. This again makes sense, because the mass is becoming more evenly spread throughout the CG cell. Still, the CGMD spectrum is significantly better than the distributed mass FEM spectrum.

The intermediate case is shown in Fig. 8 Already the distributed mass FEM is in better agreement with the MD spectra than the lumped mass FEM is. It is remarkable that cell containing as few as 8 atoms are beginning to exhibit continuum behavior. This one-two-many qualitative dependence is typical of many large- $N$ expansions, where the large- $N$ limit quickly becomes a good approximation to the real system behavior, and even for $N$ as low as two or three it is a good approximation. The 


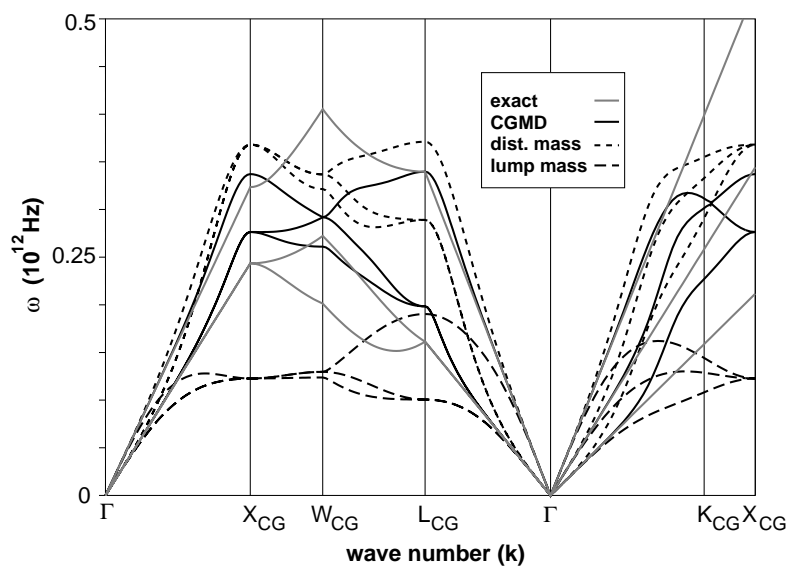

FIG. 9: The phonon spectra for solid argon on a mesh approaching the continuum limit (32768 atoms per cell) are shown as plots of wave frequencies vs. wave number along various high symmetry lines through the Brillouin zone $e^{3}$ (cf. the caption to Fig. (7). The 12 curves represent the 3 branches of the spectra for MD, CGMD and two versions of FEM, one using the conventional distributed mass matrix and one using a diagonal lumped mass matrix. With this significant level of coarse graining the CGMD spectra is again in better agreement with the MD spectra than either of the FEM spectra. Of the two FEM spectra, now the distributed mass spectra is better.

CGMD spectra are again in better agreement with the MD spectra than either of the FEM spectra are.

It should be emphasized that the shape of the FEM spectra is the same in the three plots. Continuum elasticity is scale invariant, and the changes in in FEM spectra are a simple rescaling of frequency and wave number. This scaling is clearly evident in Eq. (146) for the FEM distributed mass. The scale, $N_{\text {per }}$, enters through the prefactor $N_{p e r}^{3}$ scaling the frequency and the factor of $N_{p e r}$ in the argument of the cosine scaling the wave number. The same scaling of the wave number is present in the stiffness matrix, but its prefactor goes like $N_{\text {per }}$ rather than $N_{p e r}^{3}$. As a result, the frequency $(\sim \sqrt{K / M})$ and the wave number scale like $1 / N_{\text {per }}$. In the linear portion of the spectra near $k=0$, the two effects cancel, but the spectra are modified significantly near the zone boundary. Thus, when we discuss the comparison of the MD spectra with the FEM spectra and find better agreement with the lumped mass FEM for small cells and better agreement with distributed mass FEM for large cells, it is not that the FEM spectra are changing. The MD spectra are changing from discrete atomic behavior to continuum behavior. It is the natural scale dependence of true lattice dynamics. CGMD has this scale dependence arise naturally, as well, and so it is able to a large extent to track the changes in the MD spectra with cell size.

If we look at the differences between the spectra in more detail, we can start to understand how the under- lying physics gives rise to these differences. On all three plots, the transverse phonons are degenerate (have the same frequency) along the lines connecting the zone center and the middle of the zone faces, $\Gamma-X$ in the [100] direction and $\Gamma-L$ in the [111] direction ${ }^{63}$ This degeneracy follows from the $C_{4}$ and $C_{3}$ symmetry along those lines, respectively. The lines along the zone boundary and the $\Gamma-K$ line have reduced symmetry (at most $C_{2}$ ), and the transverse phonons are not degenerate in those cases. There are some isolated points of increased symmetry. The $W$ point is an example. One transverse phonon and the longitudinal phonon are degenerate at $W=(2,1,0) \frac{\pi}{2 a}$, as can be seen in the MD curve in Fig. 7 This happens because $W$ is the mid-point on a line between $X$ on two adjacent Brillion zones, and the longitudinal mode at one $X$ becomes the transverse mode at the other $X$, and vice-verse. The two branches must cross at the mid-point, and hence they are degenerate at $W$. This is no longer true for the MD phonons in the coarse grained systems, since $\mathrm{W}_{\mathrm{CG}}$ no longer has this mid-point property. As a result, the frequency of the longitudinal MD mode increases going from $L$ to $W(W$ is farther from $\Gamma$ ), whereas it decreases for CGMD and the two versions of FEM since the longitudinal frequency is dropping to meet the transverse frequency. This is the most pronounced discrepancy in the spectra. It is particularly bad for the lumped mass FEM as the cell becomes large. CGMD is in better agreement with the two transverse MD modes at $W$, while the distributed mass FEM, which tends to be high overall, is in better agreement with the high longitudinal MD frequency at $W$. In general, the CGMD spectra are in substantially better agreement with the MD spectra than either of the FEM spectra are.

It is not obvious, but even in the long wavelength limit (near the $\Gamma$ point), the CGMD spectrum is better than either of the two FEM spectra. The relative error for the CGMD spectrum is of order $\mathcal{O}\left(k^{4}\right)$, whereas it is $\mathcal{O}\left(k^{2}\right)$ for the two FEM cases. This improved error was demonstrated above with the formulas for the 1D frequencies, and it continues to hold in 3D. An order $\mathcal{O}\left(k^{2}\right)$ relative error is the naive expectation, since the phonon dispersion relation is linear, with the leading corrections of order $\mathcal{O}\left(k^{3}\right)$ due to symmetry. The higher order error for CGMD is due to a subtle cancellation between the mass and stiffness matrices. This cancellation can be seen from the formula for the general 1D CGMD spectra (136) and its $3 \mathrm{D}$ generalization

$$
\omega_{a b}^{2}(\mathbf{k})=\frac{1}{m} \prod_{b=1}^{3} \frac{\sum_{p_{b}=1}^{N_{p e r}} \sin ^{-4}\left(\frac{1}{2} \mathbf{a}_{b} \cdot \mathbf{k}_{p_{b}}\right)}{\sum_{p_{b}=1}^{N_{p e r}} \sin ^{-4}\left(\frac{1}{2} \mathbf{a}_{b} \cdot \mathbf{k}_{p_{b}}\right) D_{a b}^{-1}\left(\mathbf{k}_{p_{b}}\right)}(162)
$$

where $\mathbf{k}_{p_{b}}=\mathbf{k}+\frac{2 \pi p_{b}}{N_{p e r}^{b} a} \mathbf{b}_{b}$ and $\mathbf{b}_{b}$ is the reciprocal lattice basis element. Note that this formula is equivalent to Eq. (157), which is the equation we actually used to compute the CGMD spectra. The two equations differ because they make use of different expressions for the mass matrix. 
It is interesting again to compare the spectra for CGMD and R-CGMD, now for the 3D phonons. In the atomic limit $\left(N_{\text {per }}^{b}=1\right)$, the two agree with each other and with the exact MD spectrum. For coarsened lattices $\left(N_{\text {per }}^{b}>1\right)$, near the $\Gamma$ point $(\mathbf{k}=0)$, CGMD and R-CGMD are in good agreement (R-CGMD not plotted here), as was observed in the 1D case and shown in Fig. 5. We may compare the formulas for the spectra, Eq. (162) and its R-CGMD counterpart,

$$
\omega_{a b}^{2}(\mathbf{k})=\frac{1}{m} \prod_{b=1}^{3} \frac{\sum_{p_{b}=1}^{N_{p e r}} \sin ^{-4}\left(\frac{1}{2} \mathbf{a}_{b} \cdot \mathbf{k}_{p_{b}}\right) D_{a b}\left(\mathbf{k}_{p_{u}}\right)}{\sum_{p_{b}=1}^{N_{p e r}} \sin ^{-4}\left(\frac{1}{2} \mathbf{a}_{b} \cdot \mathbf{k}_{p_{b}}\right)}
$$

The two equations for the spectra may be expanded about the $\Gamma$ point, and both exhibit the improved relative error, $\mathcal{O}\left(k^{4}\right)$. Near the zone boundary, R-CGMD behaves more like the distributed mass FEM case. It is here that the relaxation physics built into CGMD has its most pronounced effect, especially at the high symmetry point $L$ on the acoustic branch, where the full CGMD error is very small.

\section{The Finite Temperature Tantalum CG Spectrum}

We have calculated the elastic wave spectrum for a variety of materials. As a second example we present results for the phonon spectra of tantalum at room temperature. Tantalum was chosen to demonstrate CGMD in a more open crystal structure (bcc) and in a system using manybody interatomic potentials. We use the Finnis-Sinclair many-body potential for tantalum ${ }^{64}$ with the improved Ackland-Thetford core repulsion ${ }^{65}$ The elastic constants for this potential are $C_{11}=266.0 \mathrm{GPa}, C_{12}=161.2 \mathrm{GPa}$, and $C_{44}=82.4 \mathrm{GPa}$. We calculate the finite temperature dynamical matrix in a conventional MD simulation consisting of 2000 atoms in a lattice of $10 \times 10 \times 10$ bcc unit cells with periodic boundary conditions. The system is equilibrated to $T=300 \pm 0.1 \mathrm{~K}$ and $P=0 \pm 10^{-3} \mathrm{GPa}$ through scaling of the box size and velocities every 100 time steps until the target temperature and pressure were attained and then an additional 5000 steps without rescaling to ensure equilibration. The equilibrium lattice constant at this temperature was found to be $3.3129 \AA$, expanded by $0.2 \%$ from the $T=0 \mathrm{~K}$ value of $3.3058 \AA$.

Subsequently, the dynamical matrix was calculated every 1000 time steps averaged over every atom in the simulation. With the Finnis-Sinclair potential it is possible to use an analytic expression for the dynamical matrix, since it is possible to take two derivatives of the energy analytically with respect to atomic displacements. The expression is given in Ref. 64. In principle we are computing an ensemble average, which we have implemented by averaging over the equivalent lattice sites of the system and over multiple time steps (relying on ergodicity for the equivalence of ensemble and temporal averages in equilibrium). In all, we have averaged over a total of

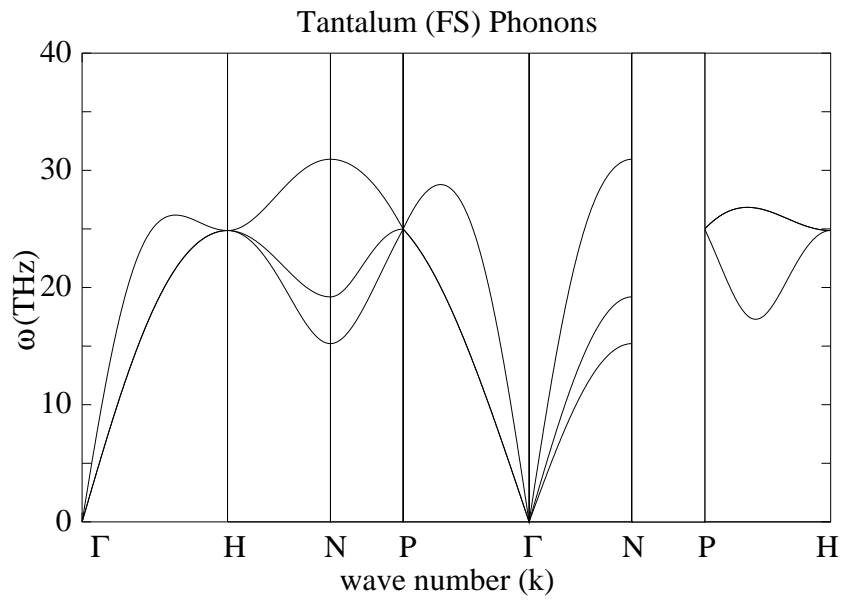

FIG. 10: The room temperature phonon spectra for solid tantalum on a mesh with no coarse graining are shown as plots of wave frequencies vs. wave number along various high symmetry lines through the Brillouin zone (for comparison to the solid argon case shown in Fig. 7). As in the case of argon, the CGMD phonon spectrum agrees exactly with the MD spectrum when the mesh is refined to the atomic limit. It is common practice to leave the gap between the second $\mathrm{N}$ and $\mathrm{P}$ points since that part of the spectrum is already represented to the left.

10 snapshots of the system and imposed the cubic $\left(\mathrm{O}_{h}\right)$ point group symmetry by averaging over the point group operations. The range of the dynamical matrix includes out to the sixth nearest neighbor shell in tantalum at $\mathrm{T}=300 \mathrm{~K}$ (the range of the pairwise functions entering the potential includes the first and second nearest neighbor shells).

The results for tantalum are very similar to those for solid argon in terms of quality. The elastic wave spectra are plotted in Figs. 10 and 11] The first figure shows the spectrum of elastic waves on a fully refined mesh. The CGMD and MD spectra agree exactly and are overlapping. The second figure shows the spectrum on a mesh consisting of 8 atoms per rhombohedral cell, as described in the argon case. Here the spectra do not agree exactly, but the results are comparable in quality to those from the argon simulations. In comparing to the corresponding argon plots (Figs. 7 and 8) it should be noted that the high symmetry $\mathrm{k}$ points are somewhat different due to the differences in the bcc and fcc crystal structures. For example, the $\Gamma-H$ line is in the [100] direction and the $\Gamma$-P line is in the [111] direction for bcc Tantalum. Again CGMD performs better than either of the two FEM models (data not shown) in reproducing the MD spectra. The many-body potential and the more open crystal structure do not have a significant impact on the quality of the results. 


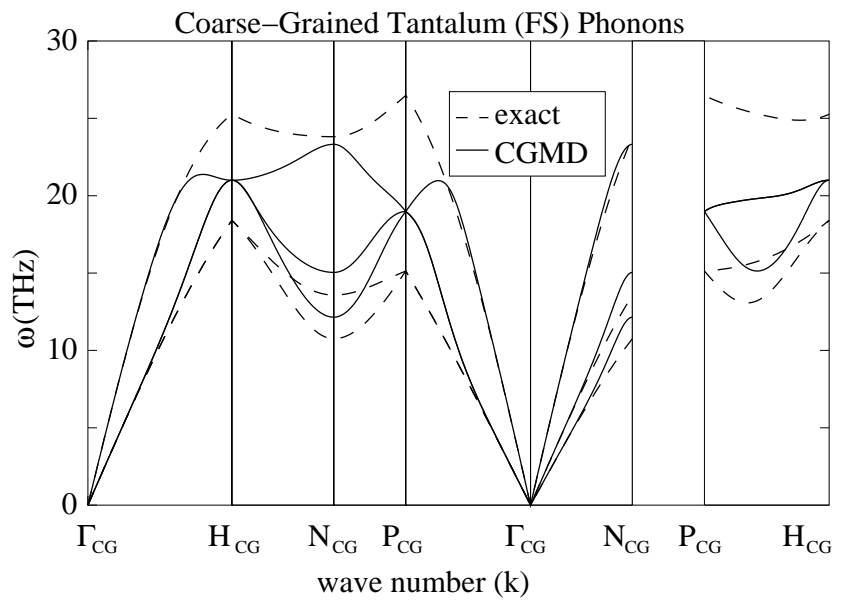

FIG. 11: The room temperature phonon spectra for solid tantalum on a mesh with 8 atoms per mesh cell are shown as plots of wave frequencies vs. wave number along various high symmetry lines through the Brillouin zone (cf. Fig. 8). As in the case of argon, the CGMD phonon spectrum agrees very well with the MD spectrum in the limit of long wavelengths (near the $\Gamma$ point), and it agrees reasonably well throughout the coarse-grained Brillouin zone.

\section{E. Dynamics and Scattering}

Most of the applications that we envision for CGMD are dynamical and have varying mesh size. For example, in studies of crack propagation it may be advantageous to introduce a coarse-grained model of the far-field regions away from the crack $\stackrel{23}{\underline{2}}$ For these applications it is important that elastic waves generated at the crack tip do not reflect from the coarse-grained region and perturb the crack propagation 66.67 .68 .69 .70 CGMD offers the chance to allow the longest wavelength modes to propagate much farther into the periphery within incurring a commensurately greater computational expense. Other coarse-grained models of the periphery, such as hybrid FEM/MD schemes, may offer the same advantage. In energy-conserving CGMD, the short wavelength modes are reflected from the CG region, but this process is sufficiently dispersive that shock waves are smoothed out and the potential wave-reflection pathologies are averted. The unphysical wave reflection may also produce a nonzero Kapitza resistance at the interface, which can lead to an unphysical temperature gradient across the interface. Of course, a stationary system started in thermal equilibrium remains at a constant, uniform temperature given a reasonable measure of temperature in the CG region, but a system driven out of equilibrium may exhibit unphysical gradients on time scales shorter than the relaxation time.

Given the potential problems associated with wave reflection, we have developed a methodology to quantify the problem. The natural measure of the ability of waves to propagate from an atomistic region into a CG region is the $S$-matrix of scattering theory, or in one dimen- sion, the transmission and reflection coefficients, $\mathcal{T}$ and $\mathcal{R}$, respectively. The basic approach to scattering problems is to look for solutions of the equations of motion of the form of an incoming plane wave and an outgoing spherical wave,

$$
\mathbf{u}(\mathbf{r}, t) \sim \frac{1}{(2 \pi)^{3 / 2}}\left[e^{i \mathbf{k} \cdot \mathbf{r}-i w \omega t}+f_{\mathbf{k}}(\hat{\mathbf{k}}) \frac{e^{i k r-i w \omega t}}{r}\right]
$$

where this asymptotic form of the displacement field holds well outside the scattering region. The $S$-matrix and the scattering cross section may be determined from $f_{\mathbf{k}}(\hat{\mathbf{k}})$. For CGMD, the scattering region is the region where the stiffness matrix differs from the MD dynamical matrix. A tremendous amount of theoretical analysis has been developed for scattering problems $\stackrel{71}{ }$ In lattice dynamics, scattering is complicated by the anisotropy of the lattice. The asymptotic form given above (164) is only applicable to isotropic scattering, but the formalism is readily generalized. To the best of our knowledge, 3D scattering cross sections have not been computed for any of the proposed solutions to the wave reflection problem.

We restrict our discussion to the one-dimensional case, for which the analysis is more straight-forward. We have calculated these scattering properties for CGMD and FEM, for comparison. The reflection coefficients are computed in the same way for both. The asymptotic region is described by harmonic MD on a regular lattice, and the normal modes are the well-known plane wave solutions. As in Eq. (164), the asymptotic form of the displacements is known for each frequency:

$$
u_{j}(t)= \begin{cases}A\left(e^{i k x_{j}-i \omega t}+r e^{-i k x_{j}-i \omega t}\right) & \text { for } \mathrm{j} \leq 1 \\ A t e^{i k x_{j}-i \omega t} & \text { for } \mathrm{j} \geq \mathrm{N}\end{cases}
$$

where the reflection and transmission coefficients are given by $R=|r|^{2}$ and $T=|t|^{2}$, respectively. We have assumed that the scattering region is contained between $x_{1}$ and $x_{N}$, in the sense that these points bound the CG region of the mesh and are separated by more than the range of the MD potential from any coarse-grained cell. This requirement guarantees that the form of $u_{j}(t)$ in the relation (165) is a strict equality and not just an asymptotic relation like (164). The wave number $k$ is determined by the frequency, $m \omega^{2}=D(k)$, where $D(k)$ is the MD dynamical matrix. The leading coefficient $A$ just determines the amplitude and is irrelevant for our purposes, so we set $A=1$. In principle, the displacement field could have components with many different frequencies, but since the problem is linear, we may restrict to a single frequency without loss of generality. Note that while we are considering the harmonic problem with a short-range MD potential, this analysis could be generalized to non-linear wave propagation and nonlinear or long-range scattering using the LSZ scattering formalism ${ }^{52}$ The incoming and outgoing waves forming the asymptotic boundary conditions (165) would need to be suitably dressed. Then the scattering cross section could be expressed in terms of the one-point irreducible 
Feynman graphs. This extension could be interesting, but the linear case will suffice for our purposes.

The equations of motion are given by

$$
M_{i j} \ddot{u}_{j}(t)=-K_{i l} u_{l}(t)
$$

and we look for solutions with angular frequency $\omega$,

$$
u_{j}(t)=e^{i k x_{j}-i \omega t}+v_{j} e^{-i \omega t}
$$

such that the asymptotic boundary conditions (165) are satisfied:

$$
v_{j}= \begin{cases}r e^{-i k x_{j}} & \text { for } \mathrm{j} \leq 1 \\ (t-1) e^{i k x_{j}} & \text { for } \mathrm{j} \geq \mathrm{N}\end{cases}
$$

where again these boundary conditions are a strict equality. The equations of motion for $v_{j}$ are

$$
\left(K_{i j}-\omega^{2} M_{i j}\right) v_{j}=-\left(K_{i j}-\omega^{2} M_{i j}\right) e^{i k x_{j}}
$$

In principle, there are many ways to solve the equations of motion (169) with the boundary conditions (168); in practice, we found this problem to be rather subtle. A similar scattering problem must have been solved before, but we have not been able to find a solution in the literature. The approach we take here is to note that we can relate the solution in the outer regions to the solution at the boundary points

$$
\begin{aligned}
v_{1-n} & =e^{i n k a} v_{1} \\
v_{N+n} & =e^{i n k a} v_{N}
\end{aligned}
$$

where $n \geq 0$. Here $a$ is the lattice constant. Using this trick, the problem is reduced to the calculation of $v_{1}, \ldots, v_{N}$ using the following $N$ equations:

$$
\begin{aligned}
\left(K_{i j}-\omega^{2} M_{i j}\right) v_{j}=-( & \left.K_{i j}-\omega^{2} M_{i j}\right) e^{i k x_{j}} \\
& +\sum_{n=1}^{\infty} K_{i(1-n)} e^{i n k a} v_{1} \\
& +\sum_{n=1}^{\infty} K_{i(N+n)} e^{i n k a} v_{N}
\end{aligned}
$$

where in practice the sums just run out to the range of the potential. The implicit sums over $j$ run from 1 to $N$. Then the scattering coefficients are determined by $\mathcal{R}=|r|^{2}$ and $\mathcal{T}=|t|^{2}$ with

$$
\begin{aligned}
r & =e^{i k x_{1}} v_{1} \\
t & =e^{-i k x_{N}} v_{N}+1
\end{aligned}
$$

which follow from Eq. (168).

In Fig. 12 we plot the reflection coefficient $\mathcal{R}(k)$ for scattering from a CG region of 72 nodes representing 652 atoms in the middle of an infinite harmonic chain of atoms. The reflection coefficients for CGMD, lumped mass FEM and distributed mass FEM are plotted. The lattice is shown in Fig. 13] The cell size increases smoothly in the $\mathrm{CG}$ region, as it should, to a maximum

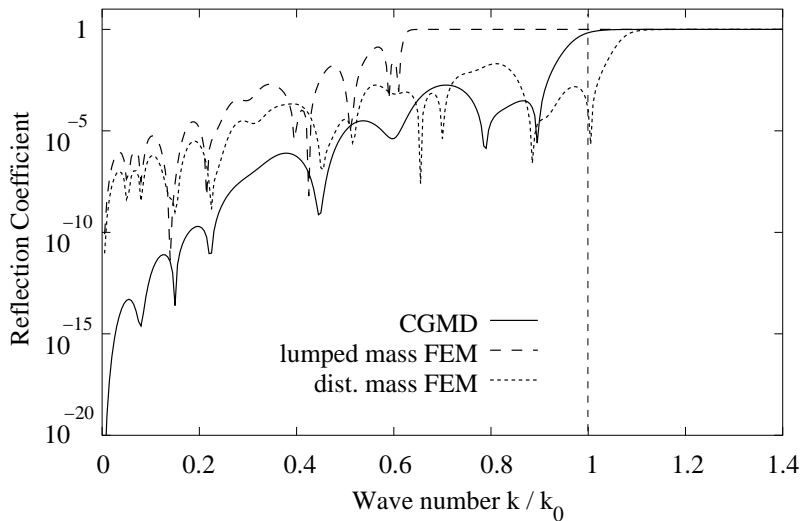

FIG. 12: A comparison of the reflection of elastic waves from a CG region in three cases: CGMD and two varieties of FEM. Note that the reflection coefficient is plotted on a log scale. A similar graph plotted on a linear scale is shown in Ref. 17. The dashed line marks the natural cutoff $\left[k_{0}=\pi /\left(N_{\max } a\right)\right]$, where $N_{\max }$ is the number of atoms in the largest cells. The bumps in the curves are scattering resonances. Note that at long wavelengths CGMD offers significantly suppressed scattering.

of $N_{\max }=20$ atoms per cell. In all three cases shown $\mathcal{R}$ becomes exponentially small in the long wavelength limit, and it goes to unity as the wavelength becomes smaller than the mesh spacing - a coarse mesh cannot support short wavelength modes. The threshold occurs approximately at $k=\pi /\left(N_{\max } a\right)$, the natural cutoff corresponding to a wavelength of $\lambda=2 N_{\max } a$. The threshold for CGMD takes place almost exactly at this point because the CGMD phonon frequencies are more accurate than those of the two versions of FEM. According to three dimensional scattering theory in the limit of wavelengths much larger than the size of the scattering region, the scattering cross section should vary like $\sigma \sim k^{4}$. This favorable transmission of long wavelengths is the well known Rayleigh scattering that gives us a blue sky. ${ }^{72}$ In these one dimensional scattering calculations, the trend is for $\mathcal{R} \sim k^{\beta}$ where the exponent $\beta$ is roughly $\beta \approx 4 \pm 1$ for FEM and $\beta \approx 8 \pm 2$ for CGMD. We hypothesize that the difference is due to the improved accuracy of CGMD at long wavelengths. Using the Born approximation, the scattering strength should go roughly like $r \sim k^{\gamma}$, where $\gamma$ is the order of the error, or $\gamma=2$ in FEM and $\gamma=4$ in CGMD. Then the reflection coefficient would go like $\mathcal{R}=|r|^{2} \sim k^{2 \gamma}$, giving $\beta \approx 4$ for $\mathrm{FEM}$ and $\beta \approx 8$ for CGMD in rough agreement with the numerical solution; however, we should stress that this hypothesis has not been proved mathematically and the resonance structure of the scattering curve leads to large uncertainties in the $\beta$ fit. If we fit to the tops of the peaks at long wavelengths in scattering from an abruptly changing mesh, the uncertainty is reduced to $\beta \approx 4 \pm 0.2$ and $8 \pm 0.2$ for FEM and CGMD, respectively.

A series of bumps is visible in each of the curves in the transmissible region. Most of these bumps were not 
FIG. 13: A plot of the mesh used for the scattering calculations. The atomic scale mesh, partially visible at the ends, extends infinitely to the right and left. The mesh is commensurate with the underlying atomic lattice, and the largest cell size is $N_{\max }=20$ atoms.

visible in plot of the reflection coefficients in Fig. 2 of Ref. 17 with a linear scale. The log scale used in Fig. 12 brings out these features in regions with extremely low scattering. These bumps are scattering resonances, wavelengths at which the scattering cross section is increased because the frequency of the incoming wave is in resonance with an internal mode of the scattering region. Of course, they are much more peaked on a linear scale, where their width is an indication of the lifetime of the state. The curvature of the peaks in the log-linear plot is low, indicating short-lived resonances. The height of the peaks is an indication of the scattering strength. If a peak were high and narrow, it would indicate a strongly scattering localized mode, which would be pathological behavior in a concurrent multiscale simulation. In general, weak scattering with broad resonances (if any) is desirable. The wave reflections in CGMD a weaker and the resonances much less strong than in FEM, although the distributed mass FEM actually has a 10\% higher threshold than CGMD because its frequencies are higher.

It is also interesting to consider the transmission coefficient, plotted in Fig. 14 for CGMD and the two versions of FEM. There is a simple relationship between the transmission and reflection coefficients, $\mathcal{T}=1-\mathcal{R}$, so in principle the calculation of one is equivalent to the other. However, because the log-linear plot brings out features near zero while suppressing features near unity, the two plots show different information. The transmission coefficient drops exponentially above the threshold, similar to quantum mechanical tunneling through a rectangular potential barrier or the transmission of evanescent waves in optics. As in those cases the transmission coefficient also decreases exponentially as the size of the scattering region is increased. One interesting feature of the transmission coefficient curves is the absence of resonances. The peaks are absent because the CG region lacks the degrees of freedom that would cause resonances at these high frequencies.

We have calculated the scattering on many different coarse-grained regions. The general features of the reflection coefficient curves remain as the mesh is varied, but the details change. One of the most pronounced changes happens if the mesh varies too abruptly. In this case, strong scattering resonances may occur near the threshold, even for CGMD, as shown in Fig.15 Note the linear scale in this plot. The mesh used for the comparison between FEM and CGMD, shown in Fig.13 was generated using a tanh function for the cell size to ensure smoothness. For comparison, we have plotted in Fig. 15 the reflection coefficient for a CG region of the same size but

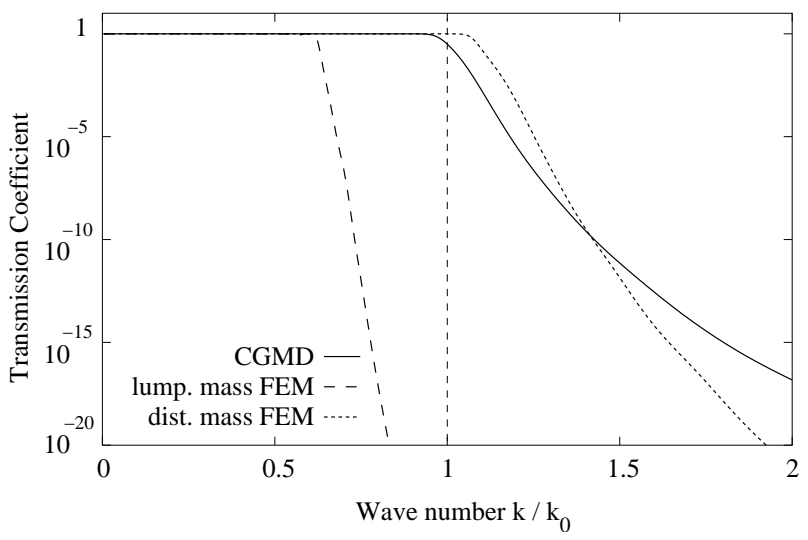

FIG. 14: A comparison of the transmission of elastic waves through a CG region in three cases: CGMD and two varieties of FEM. The dashed line marks the natural cutoff $\left[k_{0}=\pi /\left(N_{\max } a\right)\right]$. Note that the bumps evident in the plot of the reflection coefficient are absent from the transmission coefficient.

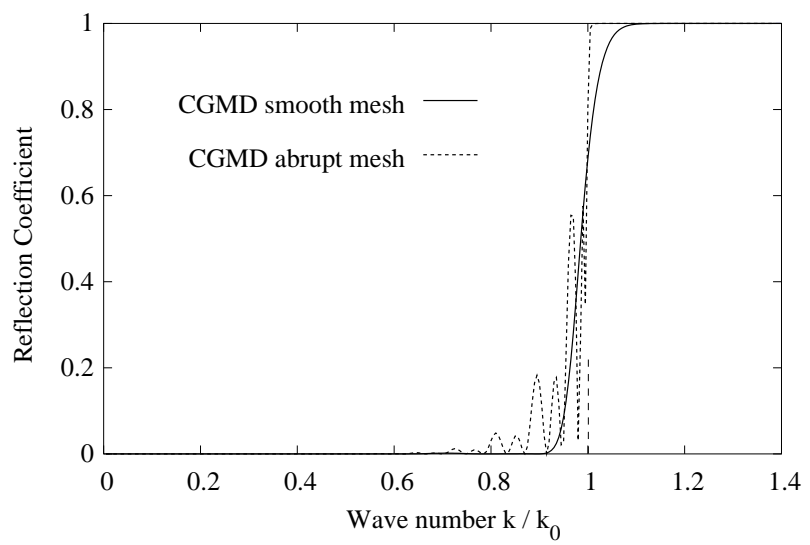

FIG. 15: A comparison of the reflection of elastic waves from a CG region for a mesh with smoothly varying cell size and one with an abrupt change in cell size, both computed in CGMD. The dashed line marks the natural cutoff $\left[k_{0}=\pi /\left(N_{\max } a\right)\right]$. Note that on the linear scale the resonances are not visible for the smooth mesh, but are quite pronounced for the abruptly changing mesh.

consisting almost exclusively of cells of size $N_{\max }=20$, and it is clear that the abrupt change in mesh size leads to much stronger resonances. The increased reflection of waves with $k$ at resonance could lead to an unphysical size scale, and smooth meshes should be used to prevent this undesirable behavior. Apart from ensuring smoothness, we have not optimized the mesh, and it may be possible to reduce the scattering further still through a more optimized mesh.

It should also be noted that the plots of wave reflection on a log-linear scale provide a sensitive test of the numerical formulation. The CGMD data plotted in Figs. 12 are not the same data plotted in Fig. 2 of Ref. 17. The data were noisy at the level of $10^{-10}$, and we ultimately recalculated the stiffness matrix using Eq. (38) in order 
to remove the noise.

The reflection coefficient provides a measure to quantify the $1 \mathrm{D}$ scattering properties of CGMD. What information does it give about the reflection problem. The actual amount of reflection in any application is the product of the elastic wave power spectrum and the reflection coefficient. Suppose the application of interest (e.g. crack propagation) can only tolerate scattering at $1 \%$ of the natural level. If $99 \%$ of the power is at wavelengths greater than $\lambda_{c}$, then there will be an acceptable level of scattering if the mesh size is less than or equal to $\lambda_{c}$.

In applications like the crack propagation problem, it may be important to consider non-linear effects as well. In the anharmonic MD crystal, waves of sufficiently large amplitude will steepen into shock waves. The wave velocity increases with the pressure, so that a wavefront with a slow rise to a higher pressure will steepen into a step-like shock wave in which the abruptness of the rise is ultimately limited by dissipative processes at the front. As a result, compressive waves that are generated at the crack tip evolve into shock waves that have a strong impact on the crack if they return to it due to the boundary conditions. The reflection coefficient is a property of the system in the small amplitude, harmonic limit, and as such does not give any information about the behavior of shock waves. Shock waves, of course, have an abrupt rise and hence have power at short wavelengths localized at the wavefront. When a shock wave is incident on the CG mesh, the short wavelengths are reflected. Since the mesh spacing increases gradually, this reflection disperses the power at the front; i.e., the shortest waves are reflected first, then the next shortest and so on. The shock wave is dispersed and much of the power flows out to the CG mesh, so the reflected wave is a low amplitude wave that does not steepen into a shock wave. Thus, while some short wavelength components are reflected, they are not shock waves and do not appear to have an appreciable impact on the processes in the MD region. The majority of the power is carried out into the $\mathrm{CG}$ region, effectively delaying its return to the MD region by the transit time across the $\mathrm{CG}$ region.

This dispersion and delay in wave reflection due to the $\mathrm{CG}$ region is the way CGMD and FEM/MD hybrid motheods solve the reflection problem. Several other solutions have been proposed that make use of absorbing boundaries. 29,53,68,69,70 Those techniques have much lower scattering of short wavelengths and hence a lower Kapitza resistance at zero temperature. They also involve considerable computer memory usage and considerable coding overhead. At this point it is clear that several approaches exist that solve the wave reflection problem in principle, but it is not yet clear which will offer the ease of use and scalability that will be demanded for widespread use in large-scale simulation.

\section{CONCLUSION}

Coarse-grained molecular dynamics provides a means of concurrently coupling MD with a coarsened description of the mechanics similar to FEM. The practical formulation relies heavily on the properties of a crystal lattice, and it is therefore suited to solids. The formulation discussed here is based on a Hamiltonian, a conserved energy for the system, and is free from ghost forces. We have applied CGMD to three dimensional systems with interatomic potentials that are many-body in nature and extend well beyond nearest neighbors. In this article, we have elaborated on how CGMD is implemented to include anharmonic (non-linear) and finite temperature effects. It has been shown that these effects are described by analytic matrix formulas that may be precomputed prior to the simulation. The formalism is useful from the point of view of both providing the means to take the calculations beyond the harmonic description and to be able to estimate the errors that are made in resorting to a harmonic model. In the process we have shown how CGMD is related to other concurrent multiscale methodologies such as the Quasicontinuum technique.

We have also reported on several calculations done with CGMD in order to understand its properties. The elastic wave spectra for solid argon and tantalum have been computed in MD, CGMD and FEM for comparison. In some cases it has been possible to derive largely analytic formulas for these spectra and to analyze their differences. In this investigation the MD spectrum has been taken as the ideal, and the CGMD and FEM models have each been formulated to give within its own framework the optimal agreement with MD. Several interesting results were found, including some reported briefly in previous publications. First, both CGMD and FEM agree well with MD in the long wavelength limit, as expected. It was shown that CGMD provides a better description, in that the error is $\mathcal{O}\left(k^{4}\right)$, than FEM with errors of the order $\mathcal{O}\left(k^{2}\right)$. Second, throughout the Brillioun zone, the CGMD errors are smaller than those of FEM, especially at certain points on the zone boundary. Of course, the CGMD spectrum is exact in the limit of one atom per mesh cell.

We have also used elastic wave spectra to examine the effect of the internal relaxation terms present in CGMD. This relaxation is the difference between forcing the atomic displacements to agree exactly with the interpolated displacement field and allowing fluctuations about the interpolated displacement field provided the interpolated field remains the best fit to the atomic displacements. We have shown how the relaxation effects can be eliminated to produce a rigid approximation to CGMD (R-CGMD), a formalism similar to the Quasicontinuum technique. We have compared the CGMD and R-CGMD elastic wave spectra and found that the two agree well in the long wavelength limit, but that CGMD provides a better description of elastic waves of short and moderate wavelengths. The internal relaxation 
is able to give a better description of the energetics of waves changing rapidly on the mesh.

Much remains to be done with CGMD, and we are actively developing the model. Many questions of numerical efficiency still need to be addressed. A controlled means of bandwidth reduction for the CGMD stiffness matrix is needed. Also an efficient and consistent treatment of wave absorption is an open challenge. We have not discussed the implementation of CGMD on parallel platforms; the decomposition of the MD region into parallel domains is straightforward, but the decomposition of the CG region is less obvious and is linked to the question of the stiffness bandwidth. Finally, the formulation of CGMD presented here provides the foundation for use in full-scale applications, a subject to which we plan to return soon.

\section{Acknowledgments}

We would like to acknowledge that much of this work was done at the Naval Research Laboratory and supported by ONR and DARPA. Support for this work was also provided by DOE/BES and the LLNL Laboratory Directed Research and Development program. This work was performed in part under the auspices of the U.S. Department of Energy by University of California, Lawrence Livermore National Laboratory under Contract W-7405-Eng-48. R. E. R. would like to thank J. Feldman for insightful discussions, and to thank A. El-Azab, B. Kraczek, B. Sadigh and many others for encouragement to finish this article.

\section{APPENDIX A: ALTERNATE DERIVATION OF CG HAMILTONIAN}

In this Appendix we compute the CG Hamiltonian using a more rigorous and straightforward, albeit laborious, approach. The choice to put this computation in an appendix rather than the main text was made because it repeats a calculation done in the main text. It gives different formulas for the mass and stiffness matrices, which are quite useful, and the positioning in an appendix is not intended to indicate that these derivations and formulas are less important than those in the main text. For this computation, as in Section IVA we will concentrate on calculating the contribution of the potential to the partition function (18):

$$
Z_{\text {pot }}\left(u_{k} ; \beta\right)=\int d u \Delta e^{-\frac{1}{2} \beta u_{\mu} D_{\mu \nu} u_{\nu}}
$$

where once again we combine the atomic and spatial indices to form the $3 N_{\text {atom-dimensional configuration space }}$ for the atoms and the $3 N_{\text {node-dimensional space for the }}$ CG displacements. Notice that we have left $\Delta$ as a product of $\delta$ functions, rather than using the Fourier repre- sentation as we did above:

$$
\Delta=\prod_{j=1}^{3 N_{\text {node }}} \delta\left(u_{j}-f_{j \mu} u_{\mu}\right)
$$

Suppose that the constraints were of a particularly simple form:

$$
\Delta^{\text {simple }}=\prod_{j=1}^{3 N_{\text {node }}} \delta\left(u_{j}-\delta_{j \mu} u_{\mu}\right)
$$

Then the evaluation of the integral would be easy. The first $3 N_{\text {node }}$ atomic displacements would not be integrated, but rather just set to the corresponding $u_{j}$ value. The remaining $3\left(N_{\text {atom }}-N_{\text {node }}\right)$ degrees of freedom would be integrated by completing the square and evaluating the Gaussian integrals. The only problem is that the constraints are not of the simple form (A3).

We must introduce some mathematical formalism to transform the constraints into a form analogous to the simple kind (A3). The approach we take in this derivation is based on an explicit factorization of the $3 N_{\text {atom- }}$ dimensional space into the direct product of the subspace spanned by the constraints and the orthogonal subspace. In order to accomplish this factorization we introduce two projection matrices in $3 N_{\text {atom-dimensional space }}$

$$
\begin{aligned}
P_{\mu \nu}^{\mathrm{CG}} & \equiv f_{j \mu}\left(f_{j \lambda} f_{k \lambda}\right)^{-1} f_{k \nu} \\
& =N_{j \mu}\left(N_{j \lambda} N_{k \lambda}\right)^{-1} N_{k \nu} \\
& =N_{j \mu} f_{j \nu} \\
P_{\mu \nu}^{\perp} & \equiv \delta_{\mu \nu}-P_{\mu \nu}^{\mathrm{CG}}
\end{aligned}
$$

where repeated indices are summed, as usual, and the inverses are $3 N_{\text {node }} \times 3 N_{\text {node }}$ matrix inverses. These are projection matrices in the sense that $P_{\mu \nu}^{C G} f_{j \nu}=f_{j \mu}$ and $P_{\mu \nu}^{\perp} f_{j \nu}=0$. Since $N_{j \mu}$ is a linear combination of $f_{k \nu}$, it likewise holds that $P_{\mu \nu}^{\mathrm{CG}} N_{j \nu}=N_{j \mu}$ and $P_{\mu \nu}^{\perp} N_{j \nu}=0$. The matrices are useful because $P^{\mathrm{CG}}$ projects onto the constrained subspace:

$$
\begin{aligned}
P_{\mu \nu}^{\mathrm{CG}} u_{\nu} & =N_{j \mu} f_{j \nu} u_{\nu} \\
& =N_{j \mu} u_{j}
\end{aligned}
$$

where we have used Eq. (A6). Thus, whenever $P^{\mathrm{CG}}$ acts on the configuration space its result depends only on the nodal displacements; it is completely independent of the unconstrained degrees of freedom. This is just what we need.

Using the fact that the two projection matrices sum to the identity, $\delta_{\mu \nu}=P_{\mu \nu}^{\perp}+P_{\mu \nu}^{\mathrm{CG}}$, we can rewrite the potential part of the partition function

$$
\begin{gathered}
Z_{\text {pot }}=\int d u \Delta e^{-\frac{1}{2} \beta u_{\mu}\left(P_{\mu \rho}^{\perp}+P_{\mu \rho}^{\mathrm{CG}}\right) D_{\rho \sigma}\left(P_{\sigma \nu}^{\perp}+P_{\sigma \nu}^{\mathrm{CG}}\right) u_{\nu}} \\
=\int d u e^{-\frac{1}{2} \beta\left(u_{\mu} D_{\mu \nu}^{\perp} u_{\nu}+2 u_{j} D_{j \mu}^{\times} u_{\mu}\right)} \times \\
\Delta e^{-\frac{1}{2} \beta u_{j} N_{j \mu} D_{\mu \nu} N_{k \nu} u_{k}}
\end{gathered}
$$


where we have introduced the notation of the orthogonal and cross components of the dynamical matrix:

$$
\begin{aligned}
D_{\mu \nu}^{\perp} & =P_{\mu \rho}^{\perp} D_{\rho \sigma} P_{\sigma \nu}^{\perp} \\
D_{j \mu}^{\times} & =P_{\mu \rho}^{\perp} D_{\rho \nu} N_{j \nu}
\end{aligned}
$$

Note that there is no $D^{C G}$ defined or used in Eq. (A10).

We can now compute the integral A10 with a slight trick. We can integrate over the constrained degrees of freedom

$$
\begin{array}{r}
Z_{\text {pot }}=C \int d^{\perp} u e^{-\frac{1}{2} \beta\left(u_{\mu} D_{\mu \nu}^{\perp} u_{\nu}+2 u_{j} D_{j \mu}^{\times} u_{\mu}\right)} \\
\times e^{-\frac{1}{2} \beta u_{j} N_{j \mu} D_{\mu \nu} N_{k \nu} u_{k}}
\end{array}
$$

where the argument of the exponential is independent of the constrained subspace, so $C$ is just a constant (independent of $\beta$ ) and hence irrelevant. Now we can restore the constrained subspace in order to make the integral easier by inserting an integral that equals unity:

$$
1=\int d^{C G} u\left(\frac{2 \pi}{\varepsilon \beta}\right)^{-3 N_{\text {node }} / 2} e^{-\frac{1}{2} \beta u_{\mu} \varepsilon P_{\mu \nu}^{\mathrm{CG}} u_{\nu}}
$$

where $\varepsilon$ is an arbitrary constant that we are free to determine below. This integral is unity because $P^{\mathrm{CG}}$ is just the identity matrix on the constrained subspace. Inserting this expression into Eq. (A13), we have

$$
\begin{aligned}
Z_{\text {pot }}=C \int d u e^{-\frac{1}{2} \beta\left[u_{\mu}\left(D_{\mu \nu}^{\perp}+\varepsilon P_{\mu \nu}^{\mathrm{CG}}\right) u_{\nu}+2 u_{j} D_{j \mu}^{\times} u_{\mu}\right]} \times \\
\left(\frac{2 \pi}{\varepsilon \beta}\right)^{-3 N_{\text {node }} / 2} e^{-\frac{1}{2} \beta u_{j} N_{j \mu} D_{\mu \nu} N_{k \nu} u_{k}}(\mathrm{~A} 15)
\end{aligned}
$$

where now we are left with an Gaussian integral over all space. Most importantly, the matrix in the Gaussian

$$
\tilde{D}_{\mu \nu}=D_{\mu \nu}^{\perp}+\varepsilon P_{\mu \nu}^{\mathrm{CG}}
$$

is non-singular provided $\varepsilon \neq 0$, as it must be since $Z_{\text {pot }}$ has been well defined at each step of the calculation.

Now we complete the square to transform Eq. A15 into a purely quadratic Gaussian integral using the shift

$$
\tilde{u}_{\mu}=u_{\mu}+\tilde{D}_{\mu \nu}^{-1} D_{j \nu}^{\times} u_{j}
$$

we find

$$
Z_{\text {pot }}=C\left(\frac{2 \pi}{\beta}\right)^{3 \delta N / 2} \frac{(\varepsilon)^{3 N_{\text {node }} / 2}}{(\operatorname{det} \tilde{D})^{1 / 2}} e^{-\frac{1}{2} \beta u_{j} K_{j k} u k}(\mathrm{~A} 18)
$$

where $\delta N=N_{\text {atom }}-N_{\text {node }}$. We find the important result that the $\mathrm{CG}$ stiffness matrix is given by

$$
K_{j k}=N_{j \mu} D_{\mu \nu} N_{k \nu}-D_{j \mu}^{\times} \tilde{D}_{\mu \nu}^{-1} D_{k \nu}^{\times}
$$

and each matrix in this expression is well defined. In principle, this expression holds for any non-zero $\varepsilon$; in practice, it is advantageous to choose $\varepsilon$ to be at the upper end of the eigenspectrum of $D_{\mu \nu}$ so that $\tilde{D}_{\mu \nu}$ is well conditioned.

The same analysis can be carried out for the mass matrix. The result is

$$
\begin{array}{rlr}
M_{j k} & =N_{j \mu} m_{\mu} N_{k \mu}-M_{j \mu}^{\times} \tilde{M}_{\mu \nu}^{-1} M_{k \nu}^{\times} \quad(\mathrm{A} 20) \\
& =m N_{j \mu} N_{k \mu} \text { for monatomic systems (A21) }
\end{array}
$$

In this case, $\varepsilon$ is chosen to be the average mass.

\section{APPENDIX B: EFFECTIVE POTENTIAL}

The CGMD formalism we have developed is an effective theory in the sense that the short wavelength modes are integrated out to determine the effective interaction of the long wavelength modes. In field theory, an effective potential is computed that is somewhat different in character. The typical approach would be to define the coarse-grained fields as an expectation value of the corresponding combination of microscopic degrees of freedom. Note that this differs from the approach we have taken in that we constrain the coarse-grained fields to equal that combination of microscopic degrees of freedom: they are identical and not an ensemble average. It is only the degrees of freedom that are integrated out that are treated as an ensemble average. We have taken this approach because at least in principle there can be bifurcation points in the trajectories of the coarse-grained degrees of freedom that would be eliminated by defining them as ensemble averages. Nevertheless, the conventional effective potential approach has a certain theoretical elegance, and it could be useful in some contexts. We will therefore give a brief discussion of the CGMD effective potential and investigate its usefulness. Hopefully, in the process we will eliminate any confusion that might arise between the two approaches.

Again we start with the Helmholtz free energy in the presence of a source $F\left(\mathbf{J}_{k}\right)$ :

$$
Z\left(\mathbf{J}_{k}\right)=e^{-\beta F\left(J_{k}\right)}=\int d u d p \exp \left(-\beta H^{M D}-\mathbf{J}_{k} \cdot N_{k \mu} \mathbf{u}_{\mu}\right)
$$

Just as in spin systems the magnetization is the derivative of the Helmholtz free energy with respect to the external field, the expectation value of the coarse-grained displacement is the derivative of this CGMD Helmholtz free energy with respect to the conjugate source:

$$
-\left.\frac{\partial F}{\partial \mathbf{J}_{k}}\right|_{\beta}=\left\langle N_{k \mu} \mathbf{u}_{\mu}\right\rangle=\mathbf{u}_{k}
$$

One could then go further and take the Legendre transform to find the Gibbs free energy $G\left(\mathbf{u}_{k}\right)$

$$
G=F+\mathbf{u}_{k} \cdot \mathbf{J}_{k}
$$

for which

$$
\left.\frac{\partial G}{\partial \mathbf{u}_{k}}\right|_{\beta}=\mathbf{J}_{k}
$$


and so is a minimum with respect to $\mathbf{u}_{k}$ at equilibrium when $\mathbf{J}_{k}=0$.

The challenge is to derive an expression for $G\left(\mathbf{u}_{k}\right)$ given that it is expressed in terms of $F\left(\mathbf{J}_{k}\right)$. A calculation of $\mathbf{J}_{k}\left(\mathbf{u}_{j}\right)$ is needed. Fortunately, the formalism is well developed in field theory ${ }^{52}$, and the result is that $G\left(\mathbf{u}_{k}\right)$ is the generating function for one-point irreducible (1PI) Feynman diagrams. The 1PI graphs are those that can- not be divided into two disconnected diagrams by breaking a single internal line. This is a subset of the connected diagrams we have considered for CGMD (cf. Fig. 2), so the effective potential approach does lead to a simplified formalism. We have not explored this model in depth. It might be interesting to do so, but we believe that the approach presented in the body of the text is more meaningful for coarse-grained solid mechanics problems.
* Corresponding author; E-mail: robert.rudd@llnl.gov

1 J. A. Moriarty, J. F. Belak, R. E. Rudd, P. Soderlind, F. H. Streitz and L. H. Yang, J. Phys.: Condens. Matter 14, 2825 (2002).

2 S. Hao, B. Moran, W. K. Liu and G. B. Olson, J. Comput.Aided Mater. Design 10, 99 (2003).

3 R. E. Rudd and J. Q. Broughton, Phys. Stat. Sol. (b) 217, 251 (2000).

${ }^{4}$ W. D. McComb, The Physics of Fluid Turbulence, (Clarendon Press, Oxford, 1990); A. A. Townsend, The Structure of Turbulent Shear Flow, (Cambridge Univ. Press, Cambridge, 1976), 2nd ed.

${ }^{5}$ E. Clementi, Philos. Trans. R. Soc. London, Ser. A 326, 445 (1988).

6 V. A. Shchukin and D. Bimberg, Rev. Mod. Phys. 71, 1125 (1995).

7 R. E. Rudd, G. A. D. Briggs, A. P. Sutton, G. MedeirosRibeiro and R. S. Williams, Phys. Rev. Lett. 90, 146101 (2003).

8 P. Liu, Y. W. Zhang and C. Lu, J. Appl. Phys. 94, 6350 (2003).

9 A. N. Cleland and M. L. Roukes, Appl. Phys. Lett. 69, 2653 (1996).

10 R. E. Rudd and J. Q. Broughton, J. Modeling and Simulation of Microsystems 1, 29 (1999).

11 R. E. Rudd and J. Q. Broughton, in Proc. DTM'99, Paris, France, B Courtois, et al, eds. (SPIE, Bellingham WA, 1999), Vol. 3680, pp. 104-13.

12 R. E. Rudd, Intl. J. on Multiscale Comput. Engin. 2, 203 (2004)

13 R. E. Rudd, Mat. Res. Soc. Symp. Proc. 677, AA1.6.1-12 (2001).

14 J. Q. Broughton, C. A. Meli, P. Vashishta, and R. K. Kalia, Phys. Rev. B 56, 611 (1997).

15 F. F. Abraham, R. Walkup, H. Gao, M. Duchaineau, T. Diaz de la Rubia, M. Seager, Proc. Natl. Acad. Sci. USA, 99, 5783 (2002).

${ }^{16}$ F. F. Abraham, J. Q. Broughton, E. Kaxiras, and N. Bernstein, Comput. in Phys. 12, 538 (1998); Europhys. Lett. (1998).

17 R. E. Rudd and J. Q. Broughton, Phys. Rev. B 58 R5893 (1998).

18 B. J. Alder and T. E. J. Wainwright, Chem. Phys. 26, 1208 (1957).

19 M. P. Allen and D. J. Tildesley, Computer Simulation of Liquids, (Clarendon Press, Oxford, 1987).

20 J. H. Argyris and S. Kelsey, Aircraft Engin. 26 and 27 (1954-1955); R.W. Clough, Proc. Second ASCE Conf. on Electronic Comput., Pittsburgh PA, pp. 345-378 (1960).

21 See for example: O. C. Zienkiewicz and R. L. Taylor, The Finite Element Method, 4th ed. (McGraw-Hill, New York,
1991), Vols. I and II;

T. J. R. Hughes, The Finite Element Method: Linear Static and Dynamic Finite Element Analysis, (Dover, Mineola, 2000).

22 S. Kohlhoff, P. Gumbsch, and H. F. Fischmeister, Philos. Mag. A 64, 851 (1991).

23 J. Q. Broughton, F. F. Abraham, N. Bernstein and E. Kaxiras, Phys. Rev. B 60, 2391 (1999).

24 E. B. Tadmor, M. Ortiz, and R. Phillips, Philos. Mag. A 73, 1529 (1996).

25 J. Knap and M. Ortiz, J. Mech. Phys. Solids 49, 1899 (2001)

26 R. E. Miller and E. B. Tadmor, J. Comput.-Aided Mater. Design 9, 203 (2002).

27 L. Dupuy, R. E. Miller, E. B. Tadmor and R. Phillips, private communication.

${ }^{28}$ W. A. Curtin and R. E. Miller, Modelling Simul. Mater. Sci. Eng. 11, R33 (2003).

29 H. S. Park, W. K. Liu, Comput. Meth. Appl. Mech. Engng. 1931733 (2004).

30 S. Curtarolo and G. Ceder, Phys. Rev. Lett. 88, 255504 (2002).

31 Z.-B. Wu, D. J. Diestler, R. Feng and X. C. Zeng, J. Chem. Phys. 119, 8013 (2003).

32 G. J. Wagner and W. K. Liu, J. Comput. Phys. 190, 249 (2003).

33 H. S. Park, E. G. Karpov, W. K. Liu, Comput. Methods Appl. Mech. Engrg. 193, 1713 (2004).

34 L. E. Shilkrot, R. E. Miller, W. A. Curtin, Phys. Rev. Lett. 89, 025501 (2002).

35 To be precise, the CGMD equations of motion agree with the atomistic equations of motion for those atoms separated by more than the range of the potential from the coarse-grained region.

36 In practice, it may be difficult to determine a priori whether the missing modes would vary adiabatically. This remains an area where additional theoretical work would be helpful.

37 L. D. Landau and E. M. Lifshitz, Theory of Elasticity, 3rd ed. (Pergamon, Oxford, 1986) Ch. 3.

38 V. B. Braginsky, V. P. Mitrofanov and I. V. Panov, Systems with Small Dissipation, (Univ. Chicago Press, Chicago, 1986).

39 We have learned recently that L. Dupuy and coworkers have derived a similar expression independently (private communication).

40 D. Chandler and P. G. J. Wolynes, Chem. Phys. 74, 4078 (1981); M. E. Tuckerman, G. J. Martyna, M. L. Klein, B. J. J. Berne, Chem. Phys. 99, 2796 (1993).

${ }^{41}$ R. E. Rudd, "The Atomic Limit of Finite Elements in the Simulation of Micro-Resonators," in Proc. of the 3rd 
Intl. Conf. on Modeling and Simulation of Microsystems (MSM2000), San Diego, Ca., March 27-29, 2000, M. Laudon and B. Romanowicz, eds. (Compuatitional Publications, Boston, 2000), pp. 465-468.

42 A. J. Williamson, L. W. Wang, and A. Zunger, Phys. Rev. B 62, 12963 (2000).

43 See for example, Appendix A of Ref. 48 .

44 The somewhat disconcerting fact that this integral is infinite is ultimately irrelevant because the multiplicative infinity cancels in the calculation of the internal energy.

45 A. C. Eringen and D. G. B. Edelen, Int. J. Eng. Sci. 10, 233 (1972).

46 S. B. Altan and E. C. Aifantis, Scr. Met. Mater. 26, 319 (1992).

47 C. Itzykson and J.-M. Drouffe, Statistical Field Theory, (Cambridge Univ. Press, Cambridge, 1991), Vol. II;

R. Kubo, M. Toda, and N. Hashitsume, Statistical Physics II, (Springer-Verlag, Berlin, 1978).

48 P. Ramond, Field Theory: A modern primer, (AddisonWesley, Redwood City, 1989), 2nd ed.

49 N. R. Werthamer, in Rare gas solids, M. L. Klein and J. A. Venables, eds. (Academic Press, New York, 1976) pp.265300 .

50 D. C. Wallace, Thermodynamics of Crystals, (Dover, New York, 1972).

${ }^{51}$ The use of $\tilde{D}_{\mu \nu}$ makes explicit the regularization that is implied in each occurrence of $D_{\mu \nu}^{-1}$ above. We return to this point in Section VID

${ }^{52}$ M. E. Peskin and D. V. Schroeder, An Introduction to Quantum Field Theory, (Addison-Wesley, New York, 1995).

53 R. E. Rudd, Mat. Res. Soc. Symp. Proc. 695, T10.2 (2002).

54 The addition of Nose-Hoover thermostats violates energy conservation in the conventional sense, but still conserves a generalized Hamiltonian.

55 R. Zwanzig, Nonequilibrium Statistical Mechanics, (Oxford Univ. Press, Oxford, 2001).
56 W. C. Swope, H. C. Andersen, P. H. Berens, K. R. Wilson, J. Chem. Phys. 76, 637 (1982).

57 M. Born and K. Huang, Dynamical Theory of Crystal Lattices, (Oxford Univ. Press, Oxford, 1998).

${ }^{58}$ K. H. Huebner, E. A. Thornton, and T. G. Byrom, The Finite Element Method for Engineers, 3rd ed., (Wiley, New York, 1995), p. 144.

59 J. G. Ergatoudis, B. M. Irons, and O. C. Zienkiewicz, Int. J. Solids Struct. 4, 31 (1968).

60 The FEM lumped mass matrix is a diagonal approximation of the true distributed mass matrix (cf. Ref. 21).

61 J. Grindlay and R. Howard, "On the Lattice Dynamics and Specific Heat of the Rare-Gas Solids," in Lattice Dynamics, Proc. Int. Conf. Copenhagen Denmark, R. F. Wallis, ed (Pergamon, Oxford, 1965), p. 129.

62 See for example, N. W. Ashcroft and N. D. Mermin, Solid State Physics, (Saunders College Press, Philadelphia, 1976), Fig. 22.13.

63 T. Inui, Y. Tanabe and Y. Onodera, Group Theory and Its Applications in Physics, (Springer-Verlag, Berlin, 1996), Section 11.6.

64 M. W. Finnis and J. E. Sinclair, Philos. Mag. 50, 45 (1984).

65 G. J. Ackland and R. Thetford, Philos. Mag. 56, 15 (1987).

66 F. F. Abraham, D. Brodbeck, R. A. Rafey, W. E. Rudge, Phys. Rev. Lett. 73, 272 (1994).

67 B. L. Holian and R. Ravelo, Phys. Rev. B 51, 11275 (1995).

68 S. A. Adelman and J. D. Doll, J. Chem. Phys. 61, 4242 (1974).

69 W. Cai, M. de Koning, V. V. Bulatov and S. Yip, Phys. Rev. Lett. 85, 3213 (2000).

70 W. E and Z. Huang, Phys. Rev. Lett. 87, 135501 (2001).

71 J. R. Taylor, Scattering Theory, (Krieger, Malabar, Florida, 1983).

72 Lord Rayleigh, Phil. Mag. XLI, 107, 274 (1871); Phil. Mag. XLVII, 375 (1899). 